\title{
On strong dynamics of compressible two-component mixture flow
}

\author{
Tomasz PIASECKI $\stackrel{*}{*}$ Yoshihiro SHIBATA $†$ and Ewelina ZATORSKA $\ddagger$
}

\begin{abstract}
We investigate a system describing the flow of a compressible two-component mixture. The system is composed of the compressible Navier-Stokes equations coupled with non-symmetric reactiondiffusion equations describing the evolution of fractional masses. We show the local existence and, under certain smallness assumptions, also the global existence of unique strong solutions in $L_{p}-L_{q}$ framework. Our approach is based on so called entropic variables which enable to rewrite the system in a symmetric form. Then, applying Lagrangian coordinates, we show the local existence of solutions applying the $L_{p}-L_{q}$ maximal regularity estimate. Next, applying exponential decay estimate we show that the solution exists globally in time provided the initial data is sufficiently close to some constants. The nonlinear estimates impose restrictions $2<p<\infty, 3<q<\infty$. However, for the purpose of generality we show the linear estimates for wider range of $p$ and $q$.
\end{abstract}

MSC Classification: 76N10, 35Q30

Keywords: compressible Navier-Stokes equations, Maxwell-Stefan equations, gaseous mixtures, regular solutions, maximal regularity, decay estimates

\section{Introduction}

The Navier-Stokes-Maxwell-Stefan equations provide a description of the multicomponent reactive flows. The system consists of compressible Navier-Stokes equations for the barycentric velocity and total density as well as the convection-diffusion equations for the constituents of the mixture. The two subsystems are coupled by the form of the pressure in the momentum equation and the form of the fluxes in the species equations. The relation between the diffusion deriving forces for the constituents and the diffusion fluxes is called the Maxwell-Stefan equations.

In this paper we are interested in analysis of a simple two-component mixture model with neglect of the heat-conduction and reactivity. The associated system of PDEs reads as follows

$$
\left\{\begin{array}{rlrl}
\partial_{t} \rho+\operatorname{div}(\rho \mathbf{u}) & =0 & & \text { in } \Omega \times(0, T), \\
\partial_{t}(\rho \mathbf{u})+\operatorname{div}(\rho \mathbf{u} \otimes \mathbf{u})-\operatorname{div} \mathbf{S}+\nabla \mathfrak{p}=0 & & \text { in } \Omega \times(0, T), \\
\partial_{t} \rho_{k}+\operatorname{div}\left(\rho_{k} \mathbf{u}\right)+\operatorname{div} \mathbf{F}_{k}=0 & & \text { in } \Omega \times(0, T),
\end{array}\right.
$$

where $\rho$ denotes the total density of the flow and is a sum of partial densities of the species $\rho=\rho_{1}+\rho_{2}$, $\mathbf{u}$ denotes the velocity vector field, $\mathfrak{p}$ denotes the pressure, $\mathbf{F}_{1}, \mathbf{F}_{2}$ the diffusion fluxes for both species and $\mathbf{S}$ denotes the stress tensor given by

$$
\mathbf{S}=\mu \mathbf{D}(\mathbf{u})+(\nu-\mu) \operatorname{div} \mathbf{u I}
$$

\footnotetext{
*Institute of Applied Mathematics and Mechanics, University of Warsaw, Banacha 2, 02-097 Warsaw, Poland.

E-mail address: tpiasecki@mimuw.edu.pl

Partially supported by Top Global University Project and polish National Science Centre Harmonia project UMO2014/14/M/ST1/00108.

${ }^{\dagger}$ Department of Mathematics and Research Institute of Science and Engineering, Waseda University, Ohkubo 3-4-1, Shinjuku-ku, Tokyo 169-8555, Japan. E-mail address: yshibata@waseda.jp

Adjunct faculty member in the Department of Mechanical Engineering and Materias Science, University of Pittsburgh.

Partially supported by JSPS Grant-in-aid for Scientific Research (A) 17H0109 and Top Global University Project.

${ }^{\ddagger}$ Department of Mathematics, University College London, Gower Street, London WC1E 6BT, United Kingdom.

E-mail address: e.zatorska@ucl.ac.uk

E.Z. has been supported by the Polish Government MNiSW research grant 2016-2019 "Iuventus Plus" no. IP2015 088874.
} 
where $\mathbf{D}(\mathbf{u})=\nabla \mathbf{u}+{ }^{\top} \nabla \mathbf{u}$ is the doubled deformation tensor. We assume the system (1.1) is supplied with the initial and boundary conditions

$$
\left\{\begin{array}{cl}
\mathbf{u}=0, \quad \mathbf{F}_{1} \cdot \mathbf{n}=\mathbf{F}_{2} \cdot \mathbf{n}=0 & \text { on } \Gamma \times(0, T), \\
\left.\left(\mathbf{u}, \rho_{1}, \rho_{2}\right)\right|_{t=0}=\left(\mathbf{u}_{0}, \rho_{10}, \rho_{20}\right) & \text { in } \Omega .
\end{array}\right.
$$

Note that assuming the constraint on the diffusion fluxes $\mathbf{F}_{1}+\mathbf{F}_{2}=0$, the species equation, when summed, give the continuity equation. Therefore wenhave $\rho_{1}=\rho-\rho_{2}$, and so, the unknowns of the system are $\rho, \mathbf{u}$, and one of the partial densities $\rho_{1}$ or $\rho_{2}$. For the derivation of system (1.1) from the kinetic theory of gases in the general multi-component, heat-conducting and reactive case we refer to the monograph of Giovangigli 19 .

In this paper we consider the mixture of ideal gases, therefore the internal pressure of the mixture is determined through the Boyle law

$$
\mathfrak{p}=\frac{\rho_{1}}{m_{1}}+\frac{\rho_{2}}{m_{2}} .
$$

Above, $m_{k}$ denotes the molar mass of the species $k$ and for simplicity, we set the gaseous constant equal to 1 . We are interested in the case when the pressure essentially depends on the densities of different species, therefore we assume

$$
m_{1} \neq m_{2} \text {. }
$$

The simplest form of the diffusion fluxes widely used in particular applications is the Fick approximation $\mathbf{F}_{k} \approx-c \nabla\left(\frac{\rho_{k}}{\rho}\right)$, see [15]. The Fick law states that the flux of a species is proportional to the gradient of the concentration of this species, and does not take into account the presence of all other components. However, in the real-word applications the cross-diffusion effects cannot be neglected, see for example [7, 46, 45, 2]. This issue can be solved by considering the so-called Maxwell-Stefan equations for multicomponent diffusion. These equations relate the diffusion velocities $\mathbf{V}_{i}$ defined as $\mathbf{F}_{i}=\rho_{i} \mathbf{V}_{i}$ and the molar and the mass fractions respectively

$$
X_{i}=\frac{\mathfrak{p}_{i}}{\mathfrak{p}}, \quad Y_{i}=\frac{\rho_{i}}{\rho}
$$

where $\mathfrak{p}_{k}=\frac{\rho_{k}}{m_{k}}$, in the implicit way:

$$
\underbrace{\nabla X_{i}-\left(Y_{i}-X_{i}\right) \nabla \log \mathfrak{p}}_{:=\mathbf{d}_{i}}=\sum_{\substack{j=1 \\ j \neq i}}^{n}\left(\frac{X_{i} X_{j}}{\mathcal{D}_{i j}}\right)\left(\mathbf{V}_{j}-\mathbf{V}_{i}\right),
$$

where $\mathcal{D}_{i j}>0$ denotes the binary diffusion coefficient, $\mathcal{D}_{i j}=\mathcal{D}_{j i}$. The Maxwell-Stefan system (1.5) was first treated by Giovangigli [17, 18, who used iterative methods to solve these equations, i.e. to find the inverse matrix that allows to characterize the fluxes as the functions of gradients of concentrations. It was proven that for positive concentrations Maxwell-Stefan relations lead to the following form of the fluxes

$$
\mathbf{F}_{k}=-\sum_{l=1}^{n} C_{k l} \mathbf{d}_{l}, \quad k=1, \ldots n
$$

where $C_{k l}$ are multicomponent flux diffusion coefficients and $\mathbf{d}_{l}=\left(d_{l}^{1}, d_{l}^{2}, d_{l}^{3}\right)$ is the species $l$ diffusion force

$$
d_{l}^{i}=\nabla_{x_{i}}\left(\frac{\mathfrak{p}_{l}}{\mathfrak{p}}\right)+\left(\frac{\mathfrak{p}_{l}}{\mathfrak{p}}-\frac{\rho_{k}}{\rho}\right) \nabla_{x_{i}} \log \mathfrak{p}=\frac{1}{\mathfrak{p}}\left(\nabla_{x_{i}} \mathfrak{p}_{l}-\frac{\rho_{l}}{\rho} \nabla_{x_{i}} \mathfrak{p}\right),
$$

appearing in the Maxwell-Stefan equations (1.5). The main properties of the flux diffusion matrix $C$ discussed in [19, Chapter 7] are

$$
C \mathcal{Y}=\mathcal{Y} C^{T}, \quad N(C)=\operatorname{lin}\{\vec{Y}\}, \quad R(C)=U^{\perp},
$$

where $\mathcal{Y}=\operatorname{diag}\left(Y_{1}, \ldots, Y_{N}\right), \vec{Y}=\left(Y_{1}, \ldots, Y_{n}\right)^{t}, N(C)$ is the nullspace of $C, R(C)$ is the range of $C$, $\vec{U}=(1, \ldots, 1)^{T}$, and $U^{\perp}$ is the orthogonal complement of $\operatorname{lin}\{\vec{U}\}$. 
In this paper we will use the explicit form (1.6). In case of two components it reduces to

$$
F_{1}=-\frac{1}{\mathfrak{p}}\left(\frac{\rho_{2}}{\rho} \nabla\left(\frac{\rho_{1}}{m_{1}}\right)-\frac{\rho_{1}}{\rho} \nabla\left(\frac{\rho_{2}}{m_{2}}\right)\right), \quad F_{2}=-F_{1} .
$$

Under the assumption (1.6), global in time strong (unique) solutions around the constant equilibrium for the Cauchy problem was proven by Giovangigli in [19]. He introduced the entropic and normal variables to symmetrize the system (1.1) and applied the Kawashima and Shizuta theory [24, 25] for symmetric hyperbolic-parabolic systems of conservation laws. For the local in time existence result to the species mass balances equations in the isobaric, isothermal case we refer to [3, see also 21]. Later on, Jüngel and Stelzer generalized this result and combined it with the entropy dissipation method to prove the global in time existence of weak solutions 23, still in the case of constant pressure and temperature. The detailed description of the method and its applicability for a range of models we refer to 22 . For the qualitative and quantitative analysis of the ternary gaseous system together with numerical simulations we refer to [7. One should note that constant pressure assumption in (1.5) not only significantly simplifies the cross-diffusion equations but basically decouples the fluid and the reactiondiffusion parts of the system (1.1). Stationary problems for compressible mixtures were considered in [49] under the assumption of Fick law and later in [20, 35, 36] with cross diffusion, however for different molar masses. Existence of weak solutions for the mixture of non-newtonian fluids has been shown in [8]. Let us also mention results on multi-phase systems [16, 26] and incompressible mixtures [27, 9, 5]. We would also like to mention the theoretical results for the systems describing the compressible reacting electrolytes [11, where the authors prove the existence of global in time weak solutions to the Nernst-Planck-Poisson model originating from the modelling approach developed by Bothe and Dreyer in the previous paper [4]. The classical mixture models in the sense of [19] were studied in the series of papers [50, 51, 30, 31, 32], where the global in time existence of weak solutions was proved without any simplification of (1.7). This was possible thanks to postulate of the so-called Bresch-Desjardins condition for the viscosity coefficients, which provides an extra estimate of the density gradient and a special form of the pressure. The last restriction was recently removed by $\mathrm{Xi}$ and Xie [52].

The global well-posedness in the framework of strong solutions for the compressible Navier-Stokes(Fourier) system under smallness assuptions on the data is already well investigated, see among others [28] in $L_{2}$ framework, [48] in $L_{p}$ setting with slip boundary condition or [38, 13, for a free boudary problem. However, for the system coupled with reaction-diffusion equations admitting cross-diffusion the issue of global well posedness of initial-boundary value problems has remained open.

The purpose of this work is to prove the global in time existence of strong solutions to the system (1.1). Our basic observation is that this system enjoys some smoothing effect when written in terms of entropic variables [19]. Its symmetric structure enables us apply $L_{p}-L_{q}$ maximal regularity estimate to show the local well posedness and exponential decay estimate to show the global well-posedness under additional smallness assumptions. The linear estimates are based on the theory of $\mathcal{R}$-bounded operators (see for instance [10, [33, [37]). The symmetrized system is derived in the next section. Afterwards we formulate our main results and discuss the structure of the remaining sections.

\section{Symmetrization and main Results.}

Since $\mathbf{F}_{1}$ and $\mathbf{F}_{2}$ are not independent, we reduce two diffusion equations to one diffusion equation introducing the normal form, see [19, Chapter 8]. Let

$$
(h, \rho)=\left(\frac{1}{m_{2}} \log \rho_{2}-\frac{1}{m_{1}} \log \rho_{1}, \quad \rho_{1}+\rho_{2}\right):=\Psi\left(\rho_{1}, \rho_{2}\right) .
$$

Notice that $\Psi: \mathbb{R}_{+} \times \mathbb{R}_{+} \rightarrow \mathbb{R} \times \mathbb{R}_{+}$is a bijection, let us denote its inverse by $\Phi$. Computing $\nabla h, \nabla \rho$ from (2.1) and solving the resulting linear system for $\nabla \rho_{1}, \nabla \rho_{2}$ we get

$$
\nabla \rho_{1}=\frac{m_{1} \rho_{1}}{m_{1} \rho_{1}+m_{2} \rho_{2}} \nabla \rho-\frac{m_{1} \rho_{1} m_{2} \rho_{2}}{m_{1} \rho_{1}+m_{2} \rho_{2}} \nabla h, \quad \nabla \rho_{2}=\frac{m_{2} \rho_{2}}{m_{1} \rho_{1}+m_{2} \rho_{2}} \nabla \rho+\frac{m_{1} \rho_{1} m_{2} \rho_{2}}{m_{1} \rho_{1}+m_{2} \rho_{2}} \nabla h .
$$


From (2.2) and the third equations in Eq. (1.1), we have

$$
\begin{aligned}
\partial_{t} h+\mathbf{u} \cdot \nabla h & =\frac{1}{m_{2} \rho_{2}} \partial_{t} \rho_{2}-\frac{1}{m_{1} \rho_{1}} \partial_{t} \rho_{1}+\frac{1}{m_{2} \rho_{2}} \mathbf{u} \cdot \nabla \rho_{2}-\frac{1}{m_{1} \rho_{1}} \mathbf{u} \cdot \nabla \rho_{1} \\
& =\frac{1}{m_{2} \rho_{2}}\left(-\rho_{2} \operatorname{div} \mathbf{u}-\operatorname{div} \mathbf{F}_{2}\right)-\frac{1}{m_{1} \rho_{1}}\left(-\rho_{1} \operatorname{div} \mathbf{u}-\operatorname{div} \mathbf{F}_{1}\right) \\
& =-\left(\frac{1}{m_{2}}-\frac{1}{m_{1}}\right) \operatorname{div} \mathbf{u}-\frac{1}{m_{2} \rho_{2}} \operatorname{div} \mathbf{F}_{2}+\frac{1}{m_{1} \rho_{1}} \operatorname{div} \mathbf{F}_{1}
\end{aligned}
$$

Since $\mathbf{F}_{1}=-\mathbf{F}_{2}$, we have

$$
\partial_{t} h+\mathbf{u} \cdot \nabla h=-\left(\frac{1}{m_{1} \rho_{1}}+\frac{1}{m_{2} \rho_{2}}\right) \operatorname{div} \mathbf{F}_{2}-\left(\frac{1}{m_{2}}-\frac{1}{m_{1}}\right) \operatorname{div} \mathbf{u},
$$

which leads to

$$
\frac{m_{1} m_{2} \rho_{1} \rho_{2}}{m_{1} \rho_{1}+m_{2} \rho_{2}}\left(\partial_{t} h+\mathbf{u} \cdot \nabla h\right)+\frac{\left(m_{1}-m_{2}\right) \rho_{1} \rho_{2}}{m_{1} \rho_{1}+m_{2} \rho_{2}}=-\operatorname{div} \mathbf{F}_{2}
$$

Moreover, noting that $m_{1}$ and $m_{2}$ are positive constants, by (1.9) and (2.2) we have

$$
\begin{aligned}
-\mathbf{F}_{2}=\mathbf{F}_{1} & =\frac{1}{\mathfrak{p}}\left(\frac{\rho_{1}}{\rho m_{2}} \nabla \rho_{2}-\frac{\rho_{2}}{\rho m_{1}} \nabla \rho_{1}\right) \\
& =\frac{1}{\mathfrak{p}}\left\{\left(\frac{\rho_{1} \rho_{2}}{\rho\left(m_{1} \rho_{1}+m_{2} \rho_{2}\right)}-\frac{\rho_{1} \rho_{2}}{\rho\left(m_{1} \rho_{1}+m_{2} \rho_{2}\right)}\right) \nabla \rho+\frac{m_{1} \rho_{1}^{2} \rho_{2}+m_{2} \rho_{1} \rho_{2}^{2}}{\rho\left(m_{1} \rho_{1}+m_{2} \rho_{2}\right)} \nabla h\right\} \\
& =\frac{\rho_{1} \rho_{2}}{\mathfrak{p} \rho} \nabla h .
\end{aligned}
$$

Combining (2.3) and (2.4) formulas gives

$$
\frac{m_{1} m_{2} \rho_{1} \rho_{2}}{m_{1} \rho_{1}+m_{2} \rho_{2}}\left(\partial_{t} h+\mathbf{u} \cdot \nabla h\right)+\frac{\left(m_{1}-m_{2}\right) \rho_{1} \rho_{2}}{m_{1} \rho_{1}+m_{2} \rho_{2}} \operatorname{div} \mathbf{u}=\operatorname{div}\left(\frac{\rho_{1} \rho_{2}}{\mathfrak{p} \rho} \nabla h\right) .
$$

By (1.4) and (2.2), we have

$$
\nabla \mathfrak{p}=\frac{1}{m_{1}} \nabla \rho_{1}+\frac{1}{m_{2}} \nabla \rho_{2}=\frac{\rho}{m_{1} \rho_{1}+m_{2} \rho_{2}} \nabla \rho+\frac{\rho_{1} \rho_{2}\left(m_{1}-m_{2}\right)}{m_{1} \rho_{1}+m_{2} \rho_{2}} \nabla h,
$$

Inserting this formula into the second equation in Eq. (1.1), we obtain

$$
\rho\left(\partial_{t} \mathbf{u}+\mathbf{u} \cdot \nabla \mathbf{u}\right)-\operatorname{div} \mathbf{S}+\frac{\rho}{m_{1} \rho_{1}+m_{2} \rho_{2}} \nabla \rho+\frac{\rho_{1} \rho_{2}\left(m_{1}-m_{2}\right)}{m_{1} \rho_{1}+m_{2} \rho_{2}} \nabla h=0 .
$$

Concerning the boundary conditions, by (2.4) the condition $\nabla \cdot \mathbf{F}_{1}=0$ is transformed to $(\nabla h) \cdot \mathbf{n}=0$. Thus, setting

$$
\Sigma_{\rho}=m_{1} \rho_{1}+m_{2} \rho_{2}, \quad \rho_{0}=\rho_{10}+\rho_{20}, \quad h_{0}=\frac{1}{m_{2}} \log \rho_{20}-\frac{1}{m_{1}} \log \rho_{10},
$$

by (2.5) and (2.6) we have the following equations for $\rho, \mathbf{u}$ and $h$ :

$$
\left\{\begin{array}{rlrl}
\partial_{t} \rho+\operatorname{div}(\rho \mathbf{u}) & =0 & & \text { in } \Omega \times(0, T), \\
\rho\left(\partial_{t} \mathbf{u}+\mathbf{u} \cdot \nabla \mathbf{u}\right)-\operatorname{div} \mathbf{S}+\frac{\rho}{\Sigma_{\rho}} \nabla \rho+\frac{\left(m_{1}-m_{2}\right) \rho_{1} \rho_{2}}{\Sigma_{\rho}} \nabla h=0 & & \text { in } \Omega \times(0, T), \\
\frac{m_{1} m_{2} \rho_{1} \rho_{2}}{\Sigma_{\rho}}\left(\partial_{t} h+\mathbf{u} \cdot \nabla h\right)+\frac{\left(m_{1}-m_{2}\right) \rho_{1} \rho_{2}}{\Sigma_{\rho}} \operatorname{div} \mathbf{u}=\operatorname{div}\left(\frac{\rho_{1} \rho_{2}}{p \rho} \nabla h\right) & & \text { in } \Omega \times(0, T), \\
\mathbf{u}=0,(\nabla h) \cdot \mathbf{n}=0 & & \text { on } \Gamma \times(0, T), \\
\left.(\rho, \mathbf{u}, h)\right|_{t=0}=\left(\rho_{0}, \mathbf{u}_{0}, h_{0}\right) & & \text { in } \Omega .
\end{array}\right.
$$


To solve Eq. (2.7) in the maximal $L_{p}-L_{q}$ regularity class, we introduce Lagrange coordinates $\{y\}$. Let $\mathbf{v}(y, t)$ be the velocity field in the Lagrange coordinates and we consider the transformation:

$$
x=y+\int_{0}^{t} \mathbf{v}(y, s) d s .
$$

Then for any differentiable function $f$ we have

$$
\partial_{t} f(t, \phi(t, y))=\partial_{t} f+\mathbf{v} \cdot \nabla_{x} f
$$

Moreover, since

$$
\frac{\partial x_{i}}{\partial y_{j}}=\delta_{i j}+\int_{0}^{t} \frac{\partial v_{i}}{\partial y_{j}}(y, s) d s
$$

where $\delta_{i j}$ are Kronecker's delta symbols, assuming

$$
\sup _{t \in(0, T)} \int_{0}^{t}\|\nabla \mathbf{v}(\cdot, s)\|_{L_{\infty}(\Omega)} d s \leq \delta
$$

with some small positive constant $\delta$, the $N \times N$ matrix $\partial x / \partial y=\left(\partial x_{i} / \partial y_{j}\right)$ has the inverse

$$
\left(\frac{\partial x_{i}}{\partial y_{j}}\right)^{-1}=\mathbf{I}+\mathbf{V}^{0}\left(\mathbf{k}_{\mathbf{v}}\right)
$$

where $\mathbf{k}_{\mathbf{v}}=\int_{0}^{t} \nabla \mathbf{v}(y, s) d s, \mathbf{I}$ is the $N \times N$ identity matrix, and $\mathbf{V}^{0}(\mathbf{k})$ is the $N \times N$ matrix of smooth functions with respect to $\mathbf{k}=\left(k_{i j} \mid i, j=1 \ldots, N\right) \in \mathbb{R}^{N^{2}}$ defined on $|\mathbf{k}|<\delta$ with $\mathbf{V}^{0}(0)=0$, where $\mathbf{k}$ is independent variables corresponding to $\mathbf{k}_{\mathbf{v}}$. Let $V_{i j}^{0}(\mathbf{k})$ be $(i, j)^{\text {th }}$ components of $\mathbf{V}^{0}(\mathbf{k})$, and then $V_{i j}^{0}(\mathbf{k})$ are smooth functions with respect to $\mathbf{k} \in B_{\delta, N^{2}}$ with $V_{i j}^{0}(0)=0$, where $B_{\delta, N^{2}}$ denotes the ball of radius $\delta$ centered at the origin in $\mathbb{R}^{N^{2}}$. We have

$$
\nabla_{x}=\left(\mathbf{I}+\mathbf{V}^{0}\left(\mathbf{k}_{\mathbf{v}}\right)\right) \nabla_{y}, \quad \frac{\partial}{\partial x_{i}}=\sum_{j=1}^{N}\left(\delta_{i j}+V_{i j}^{0}\left(\mathbf{k}_{\mathbf{v}}\right)\right) \frac{\partial}{\partial y_{j}} .
$$

Moreover, as was seen in Strömer [43], the map: $x=\Phi(y, t)$ is bijection from $\Omega$ onto $\Omega$, and so setting

$$
\mathbf{v}(y, t)=\mathbf{u}(x, t), \quad \eta(y, t)=\rho(x, t), \quad \vartheta(y, t)=h(x, t)
$$

we see that Eq. (2.7) is transformed to the following equations:

$$
\left\{\begin{aligned}
\partial_{t} \eta+\eta \operatorname{div} \mathbf{v} & =R_{1}(U) & & \text { in } \Omega \times(0, T), \\
\eta \partial_{t} \mathbf{v}-\mu \Delta \mathbf{v}-\nu \nabla \operatorname{div} \mathbf{v}+\frac{\eta}{\Sigma_{\rho}} \nabla \eta+\frac{\left(m_{1}-m_{2}\right) \rho_{1} \rho_{2}}{\Sigma_{\rho}} \nabla \vartheta & =R_{2}(U) & & \text { in } \Omega \times(0, T), \\
\frac{m_{1} m_{2} \rho_{1} \rho_{2}}{\Sigma_{\rho}} \partial_{t} \vartheta+\frac{\left(m_{1}-m_{2}\right) \rho_{1} \rho_{2}}{\Sigma_{\rho}} \operatorname{div} \mathbf{v}-\operatorname{div}\left(\frac{\rho_{1} \rho_{2}}{\mathfrak{p} \rho} \nabla \vartheta\right) & =R_{3}(U) & & \text { in } \Omega \times(0, T), \\
\mathbf{v}=0, \quad(\nabla \vartheta) \cdot \mathbf{n} & =R_{4}(U) & & \text { on } \Gamma \times(0, T), \\
\left.(\eta, \mathbf{v}, \vartheta)\right|_{t=0} & =\left(\rho_{0}, \mathbf{u}_{0}, h_{0}\right) & & \text { in } \Omega .
\end{aligned}\right.
$$

Here, $R_{1}(U), R_{2}(U), R_{3}(U)$ and $R_{4}(U)$ are nonlinear functions with respect to $U=(\eta, \mathbf{v}, \vartheta)$, which are given in Sect. 3 below.

Our main results are the following two theorems. The first one concerns the local well-posedness.

Theorem 1. Let $2<p<\infty, 3<q<\infty$ and $L>0$. Assume that $2 / p+3 / q<1$ and that $\Omega$ is a uniform $C^{3}$ domain in $\mathbb{R}^{N}(N \geq 2)$. Let $\rho_{10}(x), \rho_{20}(x)$, and $\mathbf{u}_{0}(x)$ be initial data for Eq. (1.1). Assume that there exist positive numbers $a_{1}$ and $a_{2}$ for which

$$
a_{1} \leq \rho_{10}(x), \quad \rho_{20}(x) \leq a_{2} \quad \text { for any } x \in \bar{\Omega} .
$$


Let $\left(h_{0}(x), \rho_{0}(x)\right)=\Psi\left(\rho_{10}(x), \rho_{20}(x)\right)$. Then, there exists a time $T>0$ depending on $a_{1}, a_{2}$ and $L$ such that if $\rho_{10}, \rho_{20}, \mathbf{u}_{0}$ and $h_{0}$ satisfy the condition:

$$
\left\|\nabla\left(\rho_{10}, \rho_{20}\right)\right\|_{L_{q}(\Omega)}+\left\|\mathbf{u}_{0}\right\|_{B_{q, p}^{2(1-1 / p)}(\Omega)}+\left\|h_{0}\right\|_{B_{q, p}^{2(1-1 / p)}(\Omega)} \leq L
$$

and the compatibility condition:

$$
\left.\mathbf{u}_{0}\right|_{\Gamma}=0,\left.\quad\left(\nabla h_{0}\right) \cdot \mathbf{n}\right|_{\Gamma}=0
$$

then problem (2.15) admits a unique solution $(\eta, \mathbf{v}, \vartheta)$ with

$$
\begin{gathered}
\eta-\rho_{0} \in H_{p}^{1}\left((0, T), H_{q}^{1}(\Omega)\right), \quad \mathbf{v} \in H_{p}^{1}\left((0, T), L_{q}(\Omega)^{3}\right) \cap L_{p}\left((0, T), H_{q}^{2}(\Omega)^{3}\right), \\
\vartheta \in H_{p}^{1}\left((0, T), L_{q}(\Omega)\right) \cap L_{p}\left((0, T), H_{q}^{2}(\Omega)\right)
\end{gathered}
$$

possessing the estimates:

$$
\begin{gathered}
\left\|\eta-\rho_{0}\right\|_{H_{p}^{1}\left((0, T), H_{q}^{1}(\Omega)\right)}+\left\|\partial_{t}(\mathbf{v}, \vartheta)\right\|_{L_{p}\left((0, T), L_{q}(\Omega)\right)}+\|(\mathbf{v}, \vartheta)\|_{L_{p}\left((0, T), H_{q}^{2}(\Omega)\right)} \leq C L, \\
a_{1} \leq \rho(x, t) \leq 2 a_{2}+a_{1} \quad \text { for }(x, t) \in \Omega \times(0, T), \quad \int_{0}^{T}\|\nabla \mathbf{v}(\cdot, s)\|_{L_{\infty}(\Omega)} \leq \delta .
\end{gathered}
$$

Here, $C$ is some constant independent of $L$.

The second main result gives the global well-posedness:

Theorem 2. Let $2<p<\infty, 3<q<\infty$ and $L>0$. Assume that $2 / p+3 / q<1$ and that $\Omega$ is a bounded domain whose boundary $\Gamma$ is a compact $C^{3}$ hypersurface. Let $\rho_{1 *}$ and $\rho_{2 *}$ be any positive numbers and set $\left(h_{*}, \rho_{*}\right)=\Psi\left(\rho_{1 *}, \rho_{2 *}\right) \in \mathbb{R} \times \mathbb{R}_{+}$. Then, there exists a small number $\epsilon>0$ depending on $\rho_{1 *}, \rho_{2 *}$ such that if the initial data $\left(\rho_{0}, \mathbf{u}_{0}, h_{0}\right)$ satisfy the smallness condition:

$$
\left\|\left(\rho_{10}-\rho_{1 *}, \rho_{20}-\rho_{2 *}\right)\right\|_{H_{q}^{1}(\Omega)}+\left\|\mathbf{u}_{0}\right\|_{B_{q, p}^{2(1-1 / p)}(\Omega)}+\left\|h_{0}-h_{*}\right\|_{B_{q, p}^{2(1-1 / p)}(\Omega)} \leq \epsilon
$$

and the compatibility condition (2.18) then problem (2.15) with $T=\infty$ admits a unique solution $(\eta, \mathbf{v}, \vartheta)$ with

$$
\begin{gathered}
\eta \in H_{p}^{1}\left((0, \infty), H_{q}^{1}(\Omega)\right), \quad \mathbf{v} \in H_{p}^{1}\left((0, T), L_{q}(\Omega)^{N}\right) \cap L_{p}\left((0, \infty), H_{q}^{2}(\Omega)^{N}\right), \\
\vartheta \in H_{p}^{1}\left((0, \infty), L_{q}(\Omega)\right) \cap L_{p}\left((0, T), H_{q}^{2}(\Omega)\right)
\end{gathered}
$$

possessing the estimates:

$$
\begin{aligned}
& \left\|e^{\gamma t} \nabla \eta\right\|_{L_{p}\left((0, \infty), L_{q}(\Omega)\right)}+\left\|e^{\gamma t} \partial_{t} \eta\right\|_{L_{p}\left((0, \infty), H_{q}^{1}(\Omega)\right)}+\left\|e^{\gamma t} \partial_{t}(\mathbf{v}, \vartheta)\right\|_{L_{p}\left((0, \infty), L_{q}(\Omega)\right)}+\left\|e^{\gamma t} \mathbf{v}\right\|_{L_{p}\left((0, \infty), H_{q}^{2}(\Omega)\right)} \\
& \quad+\left\|e^{\gamma t} \nabla \vartheta\right\|_{L_{p}\left((0, \infty), H_{q}^{1}(\Omega)\right)}+\left\|\left(\rho_{1}, \rho_{2}\right)-\left(\rho_{1 *}, \rho_{2 *}\right)\right\|_{L_{\infty}\left((0, \infty), H_{q}^{1}(\Omega)\right)} \leq C \epsilon, \\
& \rho_{i *} / 4 \leq \rho_{i}(x, t) \leq 4 \rho_{i *} \quad \text { in }(x, t) \in \Omega \times(0, \infty) \text { for } i=1,2, \quad \int_{0}^{T}\|\nabla \mathbf{v}(\cdot, s)\|_{L_{\infty}(\Omega)} \leq \delta
\end{aligned}
$$

for some constant $C>0$ independent of $\epsilon$.

The rest of the paper is organized as follows. In Sect.3, we derive the formulas $R_{i}(U)(i=1, \ldots, 4)$ in the right side of Eq. (2.15). In Sect.4, assuming the maximal $L_{p}-L_{q}$ theory for the linearized equations, we prove Theorem 1. In Sect.5, assuming the decay properties of solutions of the linearized equations, we prove Theorem 2. In Sect.6, we prove the maximal $L_{p}-L_{q}$ regularity for the linearized equations and in Sect.7 we prove the decay theorem for the linearized equations.

\section{Notation}

We conclude this section by summarizing the symbols used throughout the paper. We denote the sets of all complex numbers, real numbers and natural numbers by $\mathbb{C}, \mathbb{R}$, and $\mathbb{N}$, respectively, and $\mathbb{N}_{0}=\mathbb{N} \cup\{0\}$. I stands for the $N \times N$ identity matrix or the identity operator. For any multi-index $\alpha=\left(\alpha_{1}, \ldots, \alpha_{N}\right) \in \mathbb{N}_{0}^{N}$ we set $\partial_{x}^{\alpha} h=\partial_{1}^{\alpha_{1}} \cdots \partial_{N}^{\alpha_{N}} h$ with $\partial_{i}=\partial / \partial x_{i}$. In particular, for scalar functions $\theta$, vector functions 
$\mathbf{u}={ }^{\top}\left(u_{1}, \ldots, u_{N}\right)$ and $n \in \mathbb{N}_{0}$ we set $\nabla^{n} \theta=\left(\partial_{x}^{\alpha} \theta|| \alpha \mid=n\right)$ and $\nabla^{n} \mathbf{u}=\left(\nabla^{n} u_{j} \mid j=1, \ldots, N\right)$. In particular, $\nabla^{0}=\mathbf{I}, \nabla^{1}=\nabla$. For any $N$-vectors $\mathbf{a}={ }^{\top}\left(a_{1}, \ldots, a_{N}\right)$ and $\mathbf{b}={ }^{\top}\left(b_{1}, \ldots, b_{N}\right)$, let $\mathbf{a} \cdot \mathbf{b}=\langle\mathbf{a}, \mathbf{b}\rangle=\sum_{j=1}^{N} \mathbf{a}_{j} \mathbf{b}_{j}$. For any domain $G$ in $\mathbb{R}^{N}$, let $L_{q}(G), H_{q}^{m}(G)$, and $B_{q, p}^{s}(G)$ be the standard Lebesgue, Sobolev, and Besov spaces on $G$, and let $\|\cdot\|_{L_{q}(G)},\|\cdot\|_{H_{q}^{m}(G)}$, and $\|\cdot\|_{B_{q, p}^{s}(G)}$ denote their respective norms. Let $(\cdot, \cdot)_{\theta, p}$ and $(\cdot, \cdot)_{[\theta]}$ denote the real interpolation functor and complex interpolation functor, respectively. Note that $B_{q, p}^{m+\theta}(G)=\left(H_{q}^{m}(G), H_{q}^{m+1}(G)\right)_{\theta, p}$. For a Banach space $X$ with norm $\|\cdot\|_{X}$, let $X^{d}=\left\{\left(f_{1}, \ldots, f_{d}\right) \mid f_{i} \in X(i=1, \ldots, d)\right\}$, and write the norm of $X^{d}$ as simply $\|\cdot\|_{X}$, which is defined by $\|f\|_{X}=\sum_{j=1}^{d}\left\|f_{j}\right\|_{X}$ for $f=\left(f_{1}, \ldots, f_{d}\right) \in X^{d}$. Let

$$
\begin{aligned}
& \mathcal{H}_{q}(G)=\left\{F=\left(f_{1}, \mathbf{f}_{2}, f_{3}\right) \mid f_{1} \in H_{q}^{1}(G), \mathbf{f}_{2} \in L_{q}(G)^{N}, \quad f_{3} \in L_{q}(G)\right\}, \\
& \|F\|_{\mathcal{H}_{q}(G)}=\left\|f_{1}\right\|_{H_{q}^{1}(G)}+\left\|\left(\mathbf{f}_{2}, f_{3}\right)\right\|_{L_{q}(G)} \quad \text { for } F=\left(f_{1}, \mathbf{f}_{2}, f_{3}\right) \in \mathcal{H}_{q}(G), \\
& D_{q}(G)=\left\{U=(\zeta, \mathbf{v}, \vartheta) \mid \zeta \in H_{q}^{1}(G), \mathbf{v} \in H_{q}^{2}(G)^{N}, \vartheta \in H_{q}^{2}(G)\right\}, \\
& \|U\|_{D_{q}(\Omega)}=\|\zeta\|_{H_{q}^{1}(G)}+\|(\mathbf{v}, \vartheta)\|_{H_{q}^{2}(G)} \quad \text { for } U=(\zeta, \mathbf{v}, \vartheta) \in D_{q}(G), \\
& D_{p, q}(G)=\left\{U_{0}=\left(\zeta_{0}, \mathbf{v}_{0}, \vartheta_{0}\right) \mid \zeta_{0} \in H_{q}^{1}(G), \quad \mathbf{v}_{0} \in B_{q, p}^{2(1-1 / p)}(G)^{N}, \vartheta_{0} \in B_{q, p}^{2(1-1 / p)}(G)\right\}, \\
& \left\|U_{0}\right\|_{D_{p, q}(G)}=\left\|\zeta_{0}\right\|_{H_{q}^{1}(G)}+\left\|\left(\mathbf{v}_{0}, \vartheta_{0}\right)\right\|_{B_{q, p}^{2(1-1 / p)}(G)} \quad \text { for } U_{0}=\left(\zeta_{0}, \mathbf{v}_{0}, \theta_{0}\right) \in D_{p, q}(G), \\
& Y_{q}(G)=\left\{\left(h_{1}, h_{2}\right) \mid h_{1} \in L_{q}(G), \quad h_{2} \in H_{q}^{1}(G)\right\}, \quad\left\|\left(h_{1}, h_{2}\right)\right\|_{Y_{q}(G)}=\left\|h_{1}\right\|_{L_{q}(G)} \\
& \mathcal{Y}_{q}(G)=\left\{\left(F_{1}, F_{2}, F_{3}\right) \mid F_{1}, F_{2} \in L_{q}(\Omega), \quad F_{3} \in H_{q}^{1}(\Omega)\right\}, \\
& \left\|\left(F_{1}, F_{2}, F_{3}\right)\right\|_{\mathcal{Y}_{q}(G)}=\left\|\left(F_{1}, F_{2}\right)\right\|_{L_{q}(G)}+\left\|F_{3}\right\|_{H_{q}^{1}(G)} .
\end{aligned}
$$

Let $(\mathbf{u}, \mathbf{v})_{G}=\int_{G} \mathbf{u} \cdot \mathbf{v} d x$ and let $(\mathbf{u}, \mathbf{v})_{\partial G}=\int_{\partial G} \mathbf{u} \cdot \mathbf{v} d \omega$, where $d \omega$ denotes the surface element on $\partial G$.

For $1 \leq p \leq \infty, L_{p}((a, b), X)$ and $H_{p}^{m}((a, b), X)$ denote the standard Lebesgue and Sobolev spaces of $X$-valued functions defined on an interval $(a, b)$, and $\|\cdot\|_{L_{p}((a, b), X)},\|\cdot\|_{H_{p}^{m}((a, b), X)}$ denote their respective norms. Let $H_{p}^{s}(\mathbb{R}, X)$ be the standard $X$-valued Bessel potential space and $\|\cdot\|_{H_{p}^{s}(\mathbb{R}, X)}$ its norm. Let $C_{0}^{\infty}(G)$ be the set of all $C^{\infty}$ functions whose supports are compact and contained in $G$. For two Banach spaces $X$ and $Y, X+Y=\{x+y \mid x \in X, y \in Y\}, \mathcal{L}(X, Y)$ denotes the set of all bounded linear operators from $X$ into $Y$ and $\mathcal{L}(X, X)$ is written simply as $\mathcal{L}(X)$. For a domain $U$ in $\mathbb{C}$, $\operatorname{Hol}(U, \mathcal{L}(X, Y))$ denotes the set of all $\mathcal{L}(X, Y)$-valued holomorphic functions defined on $U$. Let $\Sigma_{\epsilon}=\{\lambda \in \mathbb{C} \backslash\{0\}|| \arg \lambda \mid \leq \pi-\epsilon\}$ and $\Sigma_{\epsilon, \lambda_{0}}=\left\{\lambda \in \Sigma_{\epsilon}|| \lambda \mid \geq \lambda_{0}\right\}$. Moreover, the letter $C$ denotes a generic constant and $C_{a, b, c, \ldots}$ denotes that the constant $C_{a, b, c, \cdots}$ depends on $a, b, c, \cdots$. The value of $C$ and $C_{a, b, c, \cdots}$ may change from line to line.

\section{Lagrange transformation}

In this section we rewrite all necessary differential operators under the Lagrange transformation (2.8) under the assumption (2.11). This way e obtain exact form of the right hand side of (2.15). We have

$$
\operatorname{div}_{x}=\operatorname{div}_{y}+\sum_{i, j=1}^{n} \mathbf{V}_{i j}^{0}\left(\mathbf{k}_{\mathbf{v}}\right) \frac{\partial v_{i}}{\partial y_{j}},
$$

therefore by $(2.9),(2.13)$ and $(2.14)$, we obtain $(2.15))_{1}$ with

$$
R_{1}(U)=-\eta \sum_{i, j=1}^{N} V_{i j}^{0}\left(\mathbf{k}_{\mathbf{v}}\right) \frac{\partial v_{i}}{\partial y_{j}} .
$$

Here and in the following, we set $U=(\eta, \mathbf{v}, \vartheta)$. Now we have to transform second order operators. By (2.13), we have

$$
\Delta \mathbf{u}=\sum_{k=1}^{3} \frac{\partial}{\partial x_{k}}\left(\frac{\partial \mathbf{u}}{\partial x_{k}}\right)=\sum_{k, \ell, m=1}^{3}\left(\delta_{k \ell}+V_{k l}^{0}\left(\mathbf{k}_{\mathbf{v}}\right)\right) \frac{\partial}{\partial y_{\ell}}\left(\left(\delta_{k m}+V_{k m}^{0}\left(\mathbf{k}_{\mathbf{v}}\right)\right) \frac{\partial \mathbf{v}}{\partial y_{m}}\right),
$$


and so setting

$$
\begin{aligned}
A_{2 \Delta}(\mathbf{k}) \nabla^{2} \mathbf{v} & =2 \sum_{\ell, m=1} V_{k \ell}^{0}(\mathbf{k}) \frac{\partial^{2} \mathbf{v}}{\partial y_{\ell} \partial y_{m}}+\sum_{k, \ell, m=1}^{N} V_{k \ell}^{0}(\mathbf{k}) V_{k m}^{0}(\mathbf{k}) \frac{\partial^{2} \mathbf{v}}{\partial y_{\ell} \partial y_{m}} \\
A_{1 \Delta}(\mathbf{k}) \nabla \mathbf{v} & =\sum_{\ell, m=1}^{3}\left(\nabla_{\mathbf{k}} V_{\ell m}^{0}\right)(\mathbf{k}) \int_{0}^{t}\left(\partial_{l} \nabla \mathbf{v}\right) d s \frac{\partial \mathbf{v}}{\partial y_{m}}+\sum_{k, \ell, m=1}^{3} V_{k \ell}^{0}(\mathbf{k})\left(\nabla_{\mathbf{k}} V_{k m}^{0}\right)(\mathbf{k}) \int_{0}^{t} \partial_{\ell} \nabla \mathbf{v} d s \frac{\partial \mathbf{v}}{\partial y_{m}}
\end{aligned}
$$

we have

$$
\Delta \mathbf{u}=\Delta \mathbf{v}+A_{2 \Delta}\left(\mathbf{k}_{\mathbf{v}}\right) \nabla^{2} \mathbf{v}+A_{1 \Delta}\left(\mathbf{k}_{\mathbf{v}}\right) \nabla \mathbf{v}
$$

And also, by (2.13), we have

$$
\frac{\partial}{\partial x_{j}} \operatorname{div} \mathbf{u}=\sum_{k=1}^{3}\left(\delta_{j k}+V_{j k}^{0}\left(\mathbf{k}_{\mathbf{v}}\right)\right) \frac{\partial}{\partial y_{k}}\left(\operatorname{div} \mathbf{v}+\sum_{\ell, m=1}^{3} V_{\ell m}^{0}\left(\mathbf{k}_{\mathbf{v}}\right) \frac{\partial v_{\ell}}{\partial y_{m}}\right)
$$

and so setting

$$
\begin{aligned}
& A_{2 \operatorname{div}, j}(\mathbf{k}) \nabla^{2} \mathbf{v}=\sum_{\ell, m=1}^{3} V_{\ell m}^{0}(\mathbf{k}) \frac{\partial^{2} v_{\ell}}{\partial y_{m} \partial y_{j}}+\sum_{k=1}^{3} V_{j k}^{0}(\mathbf{k}) \frac{\partial}{\partial y_{k}} \operatorname{div} \mathbf{v}+\sum_{k, \ell=1}^{3} V_{j k}^{0}(\mathbf{k}) V_{\ell m}^{0}(\mathbf{k}) \frac{\partial^{2} v_{\ell}}{\partial y_{k} \partial y_{m}}, \\
& A_{1 \operatorname{div}, j}(\mathbf{k}) \nabla \mathbf{v}=\sum_{\ell, m=1}^{3}\left(\nabla_{\mathbf{k}} V_{\ell m}^{0}\right)(\mathbf{k}) \int_{0}^{t} \partial_{j} \nabla \mathbf{v} d s \frac{\partial v_{\ell}}{\partial y_{m}}+\sum_{k, \ell, m=1}^{3} V_{j k}^{0}(\mathbf{k})\left(\nabla_{\mathbf{k}} V_{\ell m}^{0}\right)(\mathbf{k}) \int_{0}^{t} \partial_{k} \nabla \mathbf{v} d s \frac{\partial v_{\ell}}{\partial y_{m}},
\end{aligned}
$$

we have

$$
\frac{\partial}{\partial x_{j}} \operatorname{div} \mathbf{u}=\frac{\partial}{\partial y_{j}} \operatorname{div} \mathbf{v}+A_{2 \operatorname{div}, j}\left(\mathbf{k}_{\mathbf{v}}\right) \nabla^{2} \mathbf{v}+A_{1 \operatorname{div}, j}\left(\mathbf{k}_{\mathbf{v}}\right) \nabla \mathbf{v}
$$

By (2.13), we have

$$
\frac{\rho}{\Sigma_{\rho}} \nabla \rho+\frac{\left(m_{1}-m_{2}\right) \rho_{1} \rho_{2}}{\Sigma_{\rho}} \nabla h=\frac{\eta}{\Sigma_{\rho}}\left(\nabla \eta+V^{0}\left(\mathbf{k}_{\mathbf{v}}\right) \nabla \eta\right)+\frac{\left(m_{1}-m_{2}\right) \rho_{1} \rho_{2}}{\Sigma_{\rho}}\left(\nabla \vartheta+V^{0}\left(\mathbf{k}_{\mathbf{v}}\right) \nabla \vartheta\right) .
$$

Thus, noting that $\partial_{t} \mathbf{u}+\mathbf{u} \cdot \nabla \mathbf{u}=\partial_{t} \mathbf{v}$ and setting

$$
\begin{aligned}
R_{2}(U) & =\mu A_{2 \Delta}\left(\mathbf{k}_{\mathbf{v}}\right) \nabla^{2} \mathbf{v}+\mu A_{1 \Delta}\left(\mathbf{k}_{\mathbf{v}}\right) \nabla \mathbf{v}+\nu A_{2 \operatorname{div}}\left(\mathbf{k}_{\mathbf{v}}\right) \nabla^{2} \mathbf{v}+\nu A_{1 \operatorname{div}}\left(\mathbf{k}_{\mathbf{v}}\right) \nabla \mathbf{v} \\
& -\frac{\eta}{\Sigma_{\rho}} V^{0}\left(\mathbf{k}_{\mathbf{v}}\right) \nabla \eta-\frac{\left(m_{1}-m_{2}\right) \rho_{1} \rho_{2}}{\Sigma_{\rho}} V^{0}\left(\mathbf{k}_{\mathbf{v}}\right) \nabla \vartheta
\end{aligned}
$$

where $A_{i \mathrm{div}}(\mathbf{k}) \nabla^{i} \mathbf{v}={ }^{\top}\left(A_{i \mathrm{div}, 1}(\mathbf{k}) \nabla^{i} \mathbf{v}, \ldots, A_{i \mathrm{div}, N}(\mathbf{k}) \nabla^{i} \mathbf{v}\right)\left(\nabla^{1}=\nabla\right)$, we have

$$
\eta \partial_{t} \mathbf{v}-\mu \Delta \mathbf{v}-\nu \nabla \mathbf{v}+\frac{\eta}{\Sigma_{\rho}} \nabla \eta+\frac{\left(m_{1}-m_{2}\right) \rho_{1} \rho_{2}}{\Sigma_{\rho}} \nabla \vartheta=R_{2}(U) \quad \text { in } \Omega \times(0, T) .
$$

By (2.13), we have

$$
\begin{aligned}
\operatorname{div}_{x}\left(\frac{\rho_{1} \rho_{2}}{\mathfrak{p} \rho} \nabla h\right)= & \frac{\rho_{1} \rho_{2}}{\mathfrak{p} \rho}\left(\Delta \vartheta+A_{2 \Delta} \nabla^{2}\left(\mathbf{k}_{\mathbf{v}}\right) \vartheta+A_{1 \Delta}\left(\mathbf{k}_{\mathbf{v}}\right) \nabla \vartheta\right)+\nabla_{x}\left(\frac{\rho_{1} \rho_{2}}{\mathfrak{p} \rho}\right) \cdot\left(\nabla \vartheta+V^{0}\left(\mathbf{k}_{\mathbf{v}}\right) \nabla \vartheta\right) \\
= & \operatorname{div}_{y}\left(\frac{\rho_{1} \rho_{2}}{\mathfrak{p} \rho} \nabla \vartheta\right)+\frac{\rho_{1} \rho_{2}}{\mathfrak{p} \rho}\left(A_{2 \Delta}\left(\mathbf{k}_{\mathbf{v}}\right) \nabla^{2} \vartheta+A_{1 \Delta}\left(\mathbf{k}_{\mathbf{v}}\right) \nabla \vartheta\right) \\
& +\left(2 V^{0}\left(\mathbf{k}_{\mathbf{v}}\right)+\left(V^{0}\left(\mathbf{k}_{\mathbf{v}}\right)\right)^{2}\right) \nabla_{y}\left(\frac{\rho_{1} \rho_{2}}{\mathfrak{p} \rho}\right) \nabla \vartheta .
\end{aligned}
$$

Thus, noting that $\partial_{t} h+\mathbf{u} \cdot \nabla h=\partial_{t} \vartheta$ and setting

$$
\begin{aligned}
R_{3}(U)= & \frac{\rho_{1} \rho_{2}}{\mathfrak{p} \rho}\left(A_{2 \Delta}\left(\mathbf{k}_{\mathbf{v}}\right) \nabla^{2} \vartheta+A_{1 \Delta}\left(\mathbf{k}_{\mathbf{v}}\right) \nabla \vartheta\right)+\nabla\left(\frac{\rho_{1} \rho_{2}}{\mathfrak{p} \rho}\right) V^{0}\left(\mathbf{k}_{\mathbf{v}}\right) \nabla \vartheta \\
& -\frac{\left(m_{1}-m_{2}\right) \rho_{1} \rho_{2}}{\Sigma_{\rho}} \sum_{j, k=1}^{3} V_{j k}^{0}\left(\mathbf{k}_{\mathbf{v}}\right) \frac{\partial v_{j}}{\partial y_{k}}
\end{aligned}
$$


we obtain $(2.15) 3$.

Finally, by the Taylor formula we have

$$
\mathbf{n}(x)=\mathbf{n}\left(y+\int_{0}^{t} \mathbf{v}(y, s) d s\right)=\mathbf{n}(y)+\int_{0}^{1}(\nabla \mathbf{n})\left(y+\tau \int_{0}^{t} \mathbf{v}(y, s) d s\right) d \tau \int_{0}^{t} \mathbf{v}(y, s) d s,
$$

and so setting

$$
\begin{aligned}
R_{4}(U)= & -\mathbf{n}\left(y+\int_{0}^{t} \mathbf{v}(y, s) d s\right) \cdot\left(\mathbf{V}^{0}\left(\mathbf{k}_{\mathbf{v}}\right) \nabla \vartheta\right) \\
& -\left(\int_{0}^{1}(\nabla \mathbf{n})\left(y+\tau \int_{0}^{t} \mathbf{v}(y, s) d s\right) d \tau \int_{0}^{t} \mathbf{v}(y, s) d s\right) \cdot \nabla \vartheta,
\end{aligned}
$$

we obtain (2.15).

\section{Linearized problem for the local well-posedness}

Let $\rho_{10}(x), \rho_{20}(x)$ and $\mathbf{u}_{0}(x)$ be initial data for Eq. (1.1). Let $\alpha_{1}$ and $\alpha_{2}$ be postive numbers for which we assume that

$$
\alpha_{1} \leq \rho_{10}(x), \quad \rho_{20}(x) \leq \alpha_{2} \text { for any } x \in \bar{\Omega}, \quad\left\|\nabla\left(\rho_{10}, \rho_{20}\right)\right\|_{L_{r}(\Omega)} \leq \alpha_{2}
$$

where $\alpha_{1}$ and $\alpha_{2}$ are some positive constants and $3<r<\infty$. Let $\left(h_{0}(x), \rho_{0}(x)\right)=\Psi\left(\rho_{10}(x), \rho_{20}(x)\right)$, where $\Psi$ is defined in (2.1). Obviously, since $\rho_{0}(x)=\rho_{10}(x)+\rho_{20}(x)$, we have

$$
2 \alpha_{1} \leq \rho_{0}(x) \leq 2 \alpha_{2}, \quad\left|h_{0}(x)\right| \leq \alpha_{3}
$$

where $\alpha_{3}=\left(\frac{1}{m_{1}}+\frac{1}{m_{2}}\right)\left(\left|\log \alpha_{1}\right|+\left|\log \alpha_{2}\right|\right)$. We linearize Eq. (2.15) at $\left(\rho_{10}(x), \rho_{20}(x), 0\right)$. Let

$$
\begin{aligned}
& \rho=\rho_{0}(x)+\zeta, \quad \Sigma_{\rho}^{0}(x)=m_{1} \rho_{10}(x)+m_{2} \rho_{20}(x), \quad \gamma_{1}(x)=\frac{\rho_{0}(x)}{\Sigma_{\rho}^{0}(x)}, \\
& \gamma_{2}(x)=\frac{\left(m_{1}-m_{2}\right) \rho_{10}(x) \rho_{20}(x)}{\Sigma_{\rho}^{0}(x)}, \quad \gamma_{3}(x)=\frac{m_{1} m_{2} \rho_{10}(x) \rho_{20}(x)}{\Sigma_{\rho}^{0}(x)}, \\
& \gamma_{4}(x)=\frac{\rho_{10}(x) \rho_{20}(x)}{\mathfrak{p}_{0}(x) \rho_{0}(x)}, \quad \mathfrak{p}_{0}(x)=\frac{\rho_{10}(x)}{m_{1}}+\frac{\rho_{20}(x)}{m_{2}} .
\end{aligned}
$$

We then write Eq. (2.15) as

$$
\left\{\begin{aligned}
\partial_{t} \zeta+\rho_{0} \operatorname{div} \mathbf{v}=f_{1}(U) & \text { in } \Omega \times(0, T), \\
\rho_{0} \partial_{t} \mathbf{v}-\mu \Delta \mathbf{v}-\nu \nabla \operatorname{div} \mathbf{v}+\gamma_{1} \nabla \zeta+\gamma_{2} \nabla \vartheta=\mathbf{f}_{2}(U) & \text { in } \Omega \times(0, T), \\
\gamma_{3} \partial_{t} \vartheta+\gamma_{2} \operatorname{div} \mathbf{v}-\operatorname{div}\left(\gamma_{4} \nabla \vartheta\right)=f_{3}(U) & \text { in } \Omega \times(0, T), \\
\mathbf{v}=0, \quad(\nabla \vartheta) \cdot \mathbf{n}=g(U) & \text { on } \Gamma \times(0, T), \\
\left.(\zeta, \mathbf{v}, \vartheta)\right|_{t=0}=\left(0, \mathbf{u}_{0}, h_{0}\right) & \text { in } \Omega
\end{aligned}\right.
$$

where we have set $U=(\rho, \mathbf{v}, \vartheta), \rho=\rho_{0}(x)+\zeta$, and

$$
\begin{aligned}
f_{1}(U)= & R_{1}(U)-\zeta \operatorname{div} \mathbf{v} \\
\mathbf{f}_{2}(U)= & R_{2}(U)-\zeta \partial_{t} \mathbf{v}-\left(\rho_{0}+\zeta\right)\left(\frac{1}{\Sigma_{\rho}}-\frac{1}{\Sigma_{\rho}^{0}}\right) \nabla\left(\rho_{0}+\zeta\right)-\frac{\rho_{0}+\zeta}{\Sigma_{\rho}^{0}} \nabla\left(\rho_{0}\right) \\
& -\frac{\zeta}{\Sigma_{\rho}^{0}} \nabla \zeta-\left(m_{1}-m_{2}\right)\left(\frac{\rho_{1} \rho_{2}}{\Sigma_{\rho}}-\frac{\rho_{10} \rho_{20}}{\Sigma_{\rho}^{0}}\right) \nabla \vartheta \\
f_{3}(U)= & R_{3}(U)-m_{1} m_{2}\left(\frac{\rho_{1} \rho_{2}}{\Sigma_{\rho}}-\frac{\rho_{10} \rho_{20}}{\Sigma_{\rho}^{0}}\right) \partial_{t} \vartheta-\left(m_{1}-m_{2}\right)\left(\frac{\rho_{1} \rho_{2}}{\Sigma_{\rho}}-\frac{\rho_{10} \rho_{20}}{\Sigma_{\rho}^{0}}\right) \operatorname{div} \mathbf{v}
\end{aligned}
$$




$$
\begin{aligned}
& +\operatorname{div}\left(\left(\frac{\rho_{1} \rho_{2}}{\mathfrak{p} \rho}-\frac{\rho_{10} \rho_{20}}{\mathfrak{p}_{0} \rho_{0}}\right) \nabla \vartheta\right), \\
g(U)= & R_{4}(U) .
\end{aligned}
$$

In the next section we solve the system (4.4) by the Banach fixed point theorem.

\section{Local well-posedness - proof of Theorem 1}

To prove the local well-posedness, we use the maximal regularity result for the following equations:

$$
\left\{\begin{aligned}
\partial_{t} \zeta+\rho_{0}(x) \operatorname{div} \mathbf{v}=f_{1} & \text { in } \Omega \times(0, T), \\
\rho_{0}(x) \partial_{t} \mathbf{v}-\mu \Delta \mathbf{v}-\nu \nabla \operatorname{div} \mathbf{v}+\gamma_{1}(x) \nabla \zeta+\gamma_{2}(x) \nabla \vartheta=\mathbf{f}_{2} & \text { in } \Omega \times(0, T), \\
\gamma_{3}(x) \partial_{t} \vartheta+\gamma_{2}(x) \operatorname{div} \mathbf{v}-\operatorname{div}\left(\gamma_{4}(x) \nabla \vartheta\right)=f_{3} & \text { in } \Omega \times(0, T), \\
\left.\mathbf{v}\right|_{\Gamma}=0, \quad(\nabla \vartheta) \cdot \mathbf{n}=g & \text { on } \Gamma \times(0, T), \\
\left.(\zeta, \mathbf{v}, \vartheta)\right|_{t=0}=\left(\zeta_{0}, \mathbf{v}_{0}, \vartheta_{0}\right) & \text { in } \Omega .
\end{aligned}\right.
$$

Here $\gamma_{1}(x), \gamma_{2}(x), \gamma_{3}(x)$ and $\gamma_{4}(x)$ have been given in (4.3). We assume that $\rho_{10}(x), \rho_{20}(x)$ are uniformly continous functions defined on $\bar{\Omega}$ satisfying (4.1). Then we see immediately that there exist positive constants $\alpha_{3}<\alpha_{4}$ depending on $\alpha_{1}$ and $\alpha_{2}$ for which

$$
\begin{gathered}
\alpha_{3} \leq \rho_{0}(x), \gamma_{1}(x), \gamma_{3}(x), \gamma_{4}(x) \leq \alpha_{4} \text { for } x \in \bar{\Omega} \\
\left\|\nabla\left(\rho_{0}, \gamma_{1}, \gamma_{2}, \gamma_{3}, \gamma_{4}\right)\right\|_{L_{r}(\Omega)} \leq \alpha_{4} .
\end{gathered}
$$

For a Banach space $X$ with norm $\|\cdot\|_{X}$, let $H_{p}^{s}(\mathbb{R}, X)$ be a $X$ valued Bessel potential space of order $s \in(0,1)$ defined by

$$
H_{p}^{s}(\mathbb{R}, X)=\left\{f \in L_{p}(\mathbb{R}, X) \mid\|f\|_{H_{p}^{s}(\mathbb{R}, X)}<\infty\right\}, \quad\|f\|_{H_{p}^{s}(\mathbb{R}, X)}=\left\|\mathcal{F}^{-1}\left[\left(1+\tau^{2}\right)^{s / 2} \mathcal{F}[f](\tau)\right]\right\|_{L_{p}(\mathbb{R}, X)},
$$

where $\mathcal{F}$ and $\mathcal{F}^{-1}$ denote the Fourier transform and its inverse formula. The following theorems gives maximal $L_{p}-L_{q}$ regularity estimate for the system (5.1).

Theorem 3. Let $1<p, q<\infty, 2 / p+1 / q \neq 2$ and $2 / p+1 / q \neq 1$. Assume that $\Omega$ is a uniformly $C^{2}$ domain. Then, there exists a constant $\gamma_{0}$ for which the following assertion holds. Let

$$
\begin{gathered}
\zeta_{0} \in H_{q}^{1}(\Omega), \quad \mathbf{v}_{0} \in B_{q, p}^{2(1-1 / p)}(\Omega)^{3}, \quad \vartheta_{0} \in B_{q, p}^{2(1-1 / p)}(\Omega), \\
f_{1} \in L_{p}\left((0, T), H_{q}^{1}(\Omega)\right), \quad \mathbf{f}_{2} \in L_{p}\left((0, T), L_{q}(\Omega)^{3}\right), \quad f_{3} \in L_{p}\left((0, T), L_{q}(\Omega)\right), \\
e^{-\gamma t} g \in L_{p}\left(\mathbb{R}, H_{q}^{1}(\Omega)\right) \cap H_{p}^{1 / 2}\left(\mathbb{R}, L_{q}(\Omega)\right)
\end{gathered}
$$

for any $\gamma \geq \gamma_{0}$. Assume that $\mathbf{v}_{0}$ and $\vartheta_{0}$ satisfy the compatibility conditions:

$$
\left.\mathbf{v}_{0}\right|_{\Gamma}=0 \text { on } \Gamma \text { for } 2 / p+1 / q<2, \quad\left(\nabla \vartheta_{0}\right) \cdot \mathbf{n}=\left.g\right|_{t=0} \text { on } \Gamma \text { for } 2 / p+1 / q<1 .
$$

Then, problem (5.1) admits unique solutions $\zeta, \mathbf{v}$ and $\vartheta$ with

$$
\begin{gathered}
\zeta \in H_{p}^{1}\left((0, T), H_{q}^{1}(\Omega)\right), \quad \mathbf{v} \in H_{p}^{1}\left((0, T), L_{q}(\Omega)^{3}\right) \cap L_{p}\left((0, T), H_{q}^{2}(\Omega)^{3}\right), \\
\vartheta \in H_{p}^{1}\left((0, T), L_{q}(\Omega)\right) \cap L_{p}\left((0, T), H_{q}^{2}(\Omega)\right)
\end{gathered}
$$

possessing the estimate:

$$
\begin{gathered}
\|\zeta\|_{H_{p}^{1}\left((0, T), H_{q}^{1}(\Omega)\right)}+\left\|\partial_{t}(\mathbf{v}, \vartheta)\right\|_{L_{p}\left((0, T), L_{q}(\Omega)^{N}\right)}+\|(\mathbf{v}, \vartheta)\|_{L_{p}\left((0, T), H_{q}^{2}(\Omega)\right)} \\
\leq C_{\gamma} e^{\gamma T}\left\{\left\|\rho_{0}\right\|_{H_{q}^{1}(\Omega)}+\left\|\left(\mathbf{v}_{0}, \vartheta_{0}\right)\right\|_{B_{q, p}^{2(1-1 / p)}(\Omega)}+\left\|\left(f_{1}, \mathbf{f}_{2}, f_{3}\right)\right\|_{L_{p}\left((0, T), L_{q}(\Omega)\right)}\right. \\
\left.+\left\|e^{-\gamma t} g\right\|_{L_{p}\left(\mathbb{R}, H_{q}^{1}(\Omega)\right)}+\left\|e^{-\gamma t} g\right\|_{H_{p}^{1 / 2}\left(\mathbb{R}, L_{q}(\Omega)\right)}\right\}
\end{gathered}
$$

for any $\gamma \geq \gamma_{0}$, where $C$ is a constant depending on $\gamma$. 
Remark 4. All the constants appearing in Theorem 3 depend on $\alpha_{1}$ and $\alpha_{2}$.

Postponing the proof of Theorem 3, we prove Theorem 1, Let $\mathcal{H}_{T, M}$ be the underlying space for our fixed point argument, which is defined by

$$
\begin{gathered}
\mathcal{H}_{T, M}=\left\{(\zeta, \mathbf{v}, \vartheta) \mid \zeta \in H_{p}^{1}\left((0, T), H_{q}^{1}(\Omega)\right), \quad \mathbf{v} \in H_{p}^{1}\left((0, T), L_{q}(\Omega)^{3}\right) \cap L_{p}\left((0, T), H_{q}^{2}(\Omega)^{3}\right),\right. \\
\vartheta \in H_{p}^{1}\left((0, T), L_{q}(\Omega)\right) \cap L_{p}\left((0, T), H_{q}^{2}(\Omega)\right),\left.\quad(\zeta, \mathbf{v}, \vartheta)\right|_{t=0}=\left(0, \mathbf{u}_{0}, h_{0}\right) \quad \text { in } \Omega, \\
\left.[\zeta, \mathbf{v}, \vartheta]_{T}=\|\zeta\|_{H_{p}^{1}\left((0, T), H_{q}^{1}(\Omega)\right)}+\left\|\partial_{t}(\mathbf{v}, \vartheta)\right\|_{L_{p}\left((0, T), L_{q}(\Omega)\right)}+\|(\mathbf{v}, \vartheta)\|_{L_{p}\left((0, T), H_{q}^{2}(\Omega)\right)} \leq M\right\} .
\end{gathered}
$$

Here, $T$ and $M$ are positive constants determined later. Since $T$ will be chosen positive small number eventually, we may assume that $0<T \leq 1$. Moreover, by Sobolev's inequality and Hölder's inequality, we have

$$
\int_{0}^{T}\|\nabla \mathbf{v}(\cdot, t)\|_{L_{\infty}(\Omega)} d t \leq C \int_{0}^{T}\|\mathbf{v}(\cdot, t)\|_{H_{q}^{2}(\Omega)} d t \leq T^{1 / p^{\prime}}\left(\int_{0}^{T}\|\mathbf{v}(\cdot, t)\|_{H_{q}^{2}(\Omega)}^{p} d t\right)^{1 / p} \leq M T^{1 / p^{\prime}} .
$$

Thus, choosing $T>0$ so small that $M T^{1 / p^{\prime}} \leq \delta$, we may assume that the condition (2.11) holds for any $(\zeta, \mathbf{v}, \vartheta) \in \mathcal{H}_{T, M}$. Let

$$
\mathcal{I}=\left\|\nabla \rho_{0}\right\|_{H_{q}^{1}(\Omega)}+\left\|\left(\mathbf{v}, h_{0}\right)\right\|_{B_{q, p}^{2(1-1 / p)}(\Omega)},
$$

and then by (2.17) we have

$$
\mathcal{I} \leq L
$$

because $\rho_{0}(x)=\rho_{10}(x)+\rho_{20}(x)$. Let $\Psi$ be the map defined in (2.1), which is $C^{\infty}$ diffeomorphism from $\mathbb{R}_{+} \times \mathbb{R}_{+}$onto $\mathbb{R} \times \mathbb{R}_{+}$. Let $\Phi$ be its inverse map. Let $(\omega, \mathbf{w}, \varphi) \in \mathcal{H}_{T, M}$, let $U=\left(\rho_{0}(x)+\omega, \mathbf{w}, \varphi\right)$, and let $\left(\rho_{1}, \rho_{2}\right)=\Phi\left(\varphi, \rho_{0}+\omega\right)$. Since $\left.(\omega, \mathbf{w}, \varphi)\right|_{t=0}=\left(0, \mathbf{u}_{0}, h_{0}\right)$, we have

$$
\left(\rho_{10}(x), \rho_{20}(x)\right)=\left.\Phi\left(\varphi, \rho_{0}(x)+\omega\right)\right|_{t=0} .
$$

Let $R_{i}(U)$ be functions given in (3.2), (3.3), (3.4), and (3.5), where $\eta, \mathbf{v}={ }^{\top}\left(v_{1}, \ldots, v_{N}\right)$, and $\vartheta$ are replaced by $\rho_{0}+\omega, \mathbf{w}={ }^{\top}\left(w_{1}, \ldots, w_{N}\right)$, and $\varphi$. Let $(\zeta, \mathbf{v}, \vartheta)$ be a solution of Eq. (5.1) with $\zeta_{0}=0, \mathbf{v}_{0}=$ $\mathbf{u}_{0}, \vartheta_{0}=h_{0}, f_{1}=f_{1}(U), \mathbf{f}=\mathbf{f}_{2}(U), f_{3}=f_{3}(U)$ and $g=g(U)$, where $\zeta, \mathbf{v}$ and $\vartheta$ are replaced by $\omega, \mathbf{w}$ and $\varphi$, respectively.

First, we estimate $f_{1}=f_{1}(U), \mathbf{f}=\mathbf{f}_{2}(U), f_{3}=f_{3}(U)$ and $g=g(U)$. Notice that

$$
\begin{aligned}
\sup _{t \in(0, T)}\|\omega(\cdot, t)\|_{H_{q}^{1}(\Omega)} & \leq T^{1 / p^{\prime}} M \leq M, \\
\sup _{t \in(0, T)}\left\|\varphi(\cdot, t)-h_{0}\right\|_{B_{q, p}^{2(1-1 / p)}(\Omega)}+\sup _{t \in(0, T)}\left\|\mathbf{w}(\cdot, t)-\mathbf{u}_{0}\right\|_{B_{q, p}^{2(1-1 / p)}(\Omega)} & \leq C(M+L) .
\end{aligned}
$$

In fact, since $\omega(\cdot, 0)=0$, we have

$$
\left.\|\omega(\cdot, t)\|_{H_{q}^{1}(\Omega)} \leq \int_{0}^{t} \| \partial_{t} \omega\right)(\cdot, s)\left\|_{H_{q}^{1}(\Omega)} d s \leq T^{1 / p^{\prime}}\right\| \partial_{t} \omega \|_{L_{p}\left((0, T), H_{q}^{1}(\Omega)\right)} \leq T^{1 / p^{\prime}} M \leq M,
$$

where we have used the fact that $T \leq 1$ in the last step. To prove the bound for the second term in (5.6), we use the extension map $e_{T}$ defined by

$$
e_{T}[f](\cdot, t)= \begin{cases}0 & t<0, \\ f(\cdot, t) & 0<t<T, \\ f(\cdot, 2 T-t) & T<t<2 T, \\ 0 & t>2 T .\end{cases}
$$

Obviously, $e_{T}[f](\cdot, t)=f(\cdot, t)$ for $t \in(0, T)$. If $\left.f\right|_{t=0}$, then we have

$$
\partial_{t} e_{T}[f](\cdot, t)= \begin{cases}0 & t<0, \\ \left(\partial_{t} f\right)(\cdot, t) & 0<t<T, \\ -\left(\partial_{t} f\right)(\cdot, 2 T-t) & T<t<2 T, \\ 0 & t>2 T .\end{cases}
$$


Let $X$ and $Y$ be two Banach spaces such that $X$ is a dense subset of $Y$ and $X \subset Y$ is continuous, and then we know (cf. [44, p.10]) that

$$
H_{p}^{1}((0, \infty), Y) \cap L_{p}((0, \infty), X) \subset C\left([0, \infty),(X, Y)_{1 / p, p}\right)
$$

and

$$
\sup _{t \in(0, \infty)}\|u(t)\|_{(X, Y)_{1 / p, p}} \leq\left(\|u\|_{L_{p}((0, \infty), X)}^{p}+\|u\|_{H_{p}^{1}((0, \infty), Y)}^{p}\right)^{1 / p}
$$

for each $p \in(1, \infty)$, Applying this fact and using (5.7) and (5.8), we have

$$
\begin{aligned}
& \sup _{t \in(0, T)}\left\|\varphi(\cdot, t)-h_{0}\right\|_{B_{q, p}^{2(1-1 / p)}(\Omega)} \leq \sup _{t \in(0, \infty)}\left\|e_{T}\left[\varphi-h_{0}\right]\right\|_{B_{q, p}^{2(1-1 / p)}(\Omega)} \\
& \quad=\left(\left\|e_{T}\left[\varphi-h_{0}\right]\right\|_{L_{p}\left((0, \infty), H_{q}^{2}(\Omega)\right)}^{p}+\left\|e_{T}\left[\varphi-h_{0}\right]\right\|_{H_{p}^{1}\left((0, \infty), L_{q}(\Omega)\right)}^{p}\right)^{1 / p} \\
& \quad \leq C\left(\left\|\varphi-h_{0}\right\|_{L_{p}\left((0, \infty), H_{q}^{2}(\Omega)\right)}+\left\|\partial_{t} \varphi\right\|_{L_{p}\left((0, T), L_{q}(\Omega)\right)}\right) \leq C\left(M+T^{1 / p} L\right) \leq C(M+L) .
\end{aligned}
$$

Here and in the following, $C$ denotes a generic constant independent of $M, L$, and $T$. $C$ depends at most on $a_{1}$ and $a_{2}$, for which (2.16) holds. Analogously, we have the third inequality in (5.6).

Since $2 / p+3 / q<1$, we have $1+3 / q<2(1-1 / p)$, and so by Sobolev's imbedding theorem and (5.6) we have

$$
\|(\varphi, \mathbf{w})\|_{L_{\infty}\left((0, T), H_{\infty}^{1}(\Omega)\right)} \leq C M .
$$

Since $\rho_{0}(x)=\rho_{10}(x)+\rho_{20}(x)$, by (2.16) we have

$$
2 a_{1} \leq \rho_{0}(x) \leq 2 a_{2} \text { for } x \in \Omega .
$$

If we choose $T>0$ so small that $T^{1 / p^{\prime}} M \leq a_{1}$, by (5.12) and (5.6), we have

$$
a_{1} \leq \rho_{0}(x)+\omega \leq 2 a_{2}+a_{1}
$$

for all $(x, t) \in \Omega \times(0, T)$. Since $\Phi$ is a $C^{\infty}$ diffeomorphism from $\mathbb{R} \times \mathbb{R}_{+}$onto $\mathbb{R}_{+} \times \mathbb{R}_{+}$, for any compact set $A \subset \mathbb{R} \times \mathbb{R}_{+} \Phi(A)$ is a compact set in $\mathbb{R}_{+} \times \mathbb{R}_{+}$, and so by (5.13) and (5.11), there exist positive constants $a_{4}$ and $a_{5}$ depending on $a_{1}, a_{2}$, and $M$ for which

$$
a_{4} \leq \rho_{1}(x, t), \rho_{2}(x, t) \leq a_{5} \quad \text { for }(x, t) \in \Omega \times(0, T) .
$$

We now prove that

$$
\left\|\left(\rho_{1}, \rho_{2}\right)-\left(\rho_{10}, \rho_{20}\right)\right\|_{L_{\infty}\left((0, T), H_{q}^{1}(\Omega)\right)} \leq C(L+M) T^{\theta / p^{\prime}}
$$

for some $\theta \in(0,1)$. By (5.5) we have

$$
\begin{aligned}
& \sup _{t \in(0, T)}\left\|\left(\rho_{1}(\cdot, t), \rho_{2}(\cdot, t)\right)-\left(\rho_{10}(\cdot), \rho_{20}(\cdot)\right)\right\|_{L_{q}(\Omega)} \leq \int_{0}^{T}\left\|\partial_{t} \Phi\left(\varphi(\cdot, t), \rho_{0}(\cdot)+\omega(\cdot, t)\right)\right\|_{L_{q}(\Omega)} d t \\
& \quad \leq \int_{0}^{T}\left\|\Phi^{\prime}\left(\varphi(\cdot, t), \rho_{0}(\cdot)+\omega(\cdot, t)\right)\right\|_{L_{\infty}(\Omega)}\left\|\left(\partial_{t} \varphi(\cdot, t), \partial_{t} \omega(\cdot, t)\right)\right\|_{L_{q}(\Omega)} d t .
\end{aligned}
$$

By (5.11) and (5.13), we have

$$
\sup _{t \in(0, T)}\left\|\Phi^{\prime}\left(\varphi(\cdot, t), \rho_{0}(\cdot)+\omega(\cdot, t)\right)\right\|_{L_{\infty}(\Omega)} \leq a_{6}
$$

for some positive constant $a_{6}$ depending on $a_{1}, a_{2}, M$ but independent of $T$. Thus, by (5.16) we have

$$
\begin{aligned}
\sup _{t \in(0, T)}\left\|\left(\rho_{1}(\cdot, t), \rho_{2}(\cdot, t)\right)-\left(\rho_{10}(\cdot), \rho_{20}(\cdot)\right)\right\|_{L_{q}(\Omega)} & \leq a_{6} \int_{0}^{T}\left\|\left(\partial_{t} \varphi(\cdot, t), \partial_{t} \omega(\cdot, t)\right)\right\|_{L_{q}(\Omega)} d t \\
& \leq a_{6} T^{1 / p^{\prime}}\left\|\partial_{t}(\varphi, \omega)\right\|_{L_{p}\left((0, T), L_{q}(\Omega)\right)} \leq a_{6} M T^{1 / p^{\prime}} .
\end{aligned}
$$


Moreover, by (2.17) and (5.4) we have

$$
\begin{aligned}
& \left\|\nabla\left(\rho_{1}(\cdot, t), \rho_{2}(\cdot, t)\right)-\left(\rho_{10}(\cdot), \rho_{20}(\cdot)\right)\right\|_{L_{q}(\Omega)} \\
& \quad \leq \| \Phi^{\prime}\left(\varphi(\cdot, t), \rho_{0}(\cdot)+\omega(\cdot, t)\left\|_{L_{\infty}(\Omega)}\right\|\left(\nabla \varphi(\cdot, t), \nabla \rho_{0}(\cdot)+\nabla \omega(\cdot, t)\right)\left\|_{L_{q}(\Omega)}+\right\| \nabla\left(\rho_{10}, \rho_{20}\right) \|_{L_{q}(\Omega)}\right. \\
& \quad \leq a_{6}\left(\|\nabla \varphi(\cdot, t)\|_{L_{q}(\Omega)}+\|\nabla \omega(\cdot, t)\|_{L_{q}(\Omega)}\right)+a_{6}\left\|\nabla \rho_{0}\right\|_{L_{q}(\Omega)}+\left\|\nabla\left(\rho_{10}, \rho_{20}\right)\right\|_{L_{q}(\Omega)} .
\end{aligned}
$$

Thus, by (5.6)

$$
\sup _{t \in(0, T)}\left\|\nabla\left\{\left(\rho_{1}(\cdot, t), \rho_{2}(\cdot, t)\right)-\left(\rho_{10}(\cdot), \rho_{20}(\cdot)\right)\right\}\right\|_{L_{q}(\Omega)} \leq C(L+M) .
$$

Since $W_{q}^{3 / q+\epsilon}(\Omega) \subset L_{\infty}(\Omega)$ with some small $\epsilon$ for which $3 / q+\epsilon<1$ and this inclusion is continuous as follows from Sobolev's imbedding theorem, by real interpolation theorem

$$
\begin{aligned}
& \sup _{t \in(0, T)}\left\|\left(\rho_{1}(\cdot, t), \rho_{2}(\cdot, t)\right)-\left(\rho_{10}(\cdot), \rho_{20}(\cdot)\right)\right\|_{L_{\infty}(\Omega)} \\
& \quad \leq\left(\sup _{0 \in(0, T)}\left\|\left(\rho_{1}(\cdot, t), \rho_{2}(\cdot, t)\right)-\left(\rho_{10}(\cdot), \rho_{20}(\cdot)\right)\right\|_{L_{q}(\Omega)}\right)^{\theta} \\
& \quad \times\left(\sup _{0 \in(0, T)}\left\|\left(\rho_{1}(\cdot, t), \rho_{2}(\cdot, t)\right)-\left(\rho_{10}(\cdot), \rho_{20}(\cdot)\right)\right\|_{H_{q}^{1}(\Omega)}\right)^{1-\theta} \leq C(M+L) T^{\theta / p^{\prime}}
\end{aligned}
$$

with $\theta=1-(3 / q+\epsilon) \in(0,1)$. By (5.20), (5.14), and (2.16), we have

$$
\begin{array}{r}
\left\|\frac{1}{\Sigma_{\rho}}-\frac{1}{\Sigma_{\rho}^{0}}\right\|_{L_{\infty}\left((0, T), L_{\infty}(\Omega)\right)}+\left\|\frac{\rho_{1} \rho_{2}}{\Sigma_{\rho}}-\frac{\rho_{10} \rho_{20}}{\Sigma_{\rho}^{0}}\right\|_{L_{\infty}\left((0, T), L_{\infty}(\Omega)\right)}+\left\|\frac{\rho_{1} \rho_{2}}{\mathfrak{p} \rho}-\frac{\rho_{10} \rho_{20}}{\mathfrak{p}_{0} \rho_{0}}\right\|_{L_{\infty}\left((0, T), L_{\infty}(\Omega)\right)} \\
\leq C(M+L) T^{\theta / p^{\prime}} .
\end{array}
$$

Moreover, by (5.19) we have

$$
\sup _{t \in(0, T)}\left\|\nabla\left(\rho_{1}(\cdot, t), \rho_{2}(\cdot, t)\right)\right\|_{L_{q}(\Omega)} \leq C(L+M),
$$

and so by (5.14) and (2.16) we get

$$
\left\|\nabla\left(\frac{\rho_{1} \rho_{2}}{\mathfrak{p} \rho}-\frac{\rho_{10} \rho_{20}}{\mathfrak{p}_{0} \rho_{0}}\right)\right\|_{L_{\infty}\left((0, T), L_{q}(\Omega)\right)} \leq C(M+L) .
$$

Using (5.11), (5.6), (5.21), and (5.22), we conclude

$$
\begin{aligned}
& \left\|\left(\rho_{0}+\omega\right)\left(\frac{1}{\Sigma_{\rho}}-\frac{1}{\Sigma_{\rho}^{0}}\right) \nabla\left(\rho_{0}+\omega\right)\right\|_{L_{p}\left((0, T), L_{q}(\Omega)\right)} \\
& \quad \leq C\left\|\rho_{0}+\omega\right\|_{L_{\infty}\left((0, T), H_{q}^{1}(\Omega)\right)}^{2} T^{1 / p}(M+L) T^{\theta / p^{\prime}} \leq C(M+L)^{3} T^{\left(1 / p+\theta / p^{\prime}\right)} ; \\
& \left\|\frac{\rho_{0}+\omega}{\Sigma_{\rho}^{0}} \nabla \rho_{0}\right\|_{L_{p}\left((0, \infty), L_{q}(\Omega)\right)} \leq C(M+L) L T^{1 / p} ; \\
& \left\|\frac{\omega}{\Sigma_{\rho}^{0}} \nabla \omega\right\|_{L_{p}\left((0, T), L_{q}(\Omega)\right)} \leq C\|\omega\|_{L_{\infty}\left((0, T), H_{q}^{1}(\Omega)\right)} T^{1 / p} \leq C L^{2} T^{1 / p} ; \\
& \left\|\left(\frac{\rho_{1} \rho_{2}}{\Sigma_{\rho}}-\frac{\rho_{10} \rho_{20}}{\Sigma_{\rho}^{0}}\right) \nabla \varphi\right\|_{L_{p}\left((0, T), L_{q}(\Omega)\right)} \leq C(M+L) L T^{\left(\theta / p^{\prime}+1 / p\right) ;} \\
& \left\|\left(\frac{\rho_{1} \rho_{2}}{\Sigma_{\rho}}-\frac{\rho_{10} \rho_{20}}{\Sigma_{\rho}^{0}}\right) \partial_{t} \omega\right\|_{L_{p}\left((0, T), L_{q}(\Omega)\right)} \leq C(M+L) T^{\theta / p^{\prime}}\left\|\partial_{t} \omega\right\|_{L_{p}\left((0, T), L_{q}(\Omega)\right)} \leq C M(M+L) T^{\theta / p^{\prime}} ; \\
& \left\|\left(\frac{\rho_{1} \rho_{2}}{\Sigma_{\rho}}-\frac{\rho_{10} \rho_{20}}{\Sigma_{\rho}^{0}}\right) \operatorname{div} \mathbf{w}\right\|_{L_{p}\left((0, T), L_{q}(\Omega)\right)} \\
& \quad \leq C(M+L) T^{\theta / p^{\prime}} T^{1 / p}\|\mathbf{w}\|_{L_{\infty}\left((0, T), H_{q}^{1}(\Omega)\right)} \leq C(M+L)^{2} T^{\left(\theta / p^{\prime}+1 / p\right)} ; \\
& \left\|\operatorname{div}\left(\left(\frac{\rho_{1} \rho_{2}}{\mathfrak{p} \rho}-\frac{\rho_{10} \rho_{20}}{\mathfrak{p}_{0} \rho_{0}}\right) \nabla \varphi\right)\right\|_{L_{p}\left((0, T), L_{q}(\Omega)\right)}
\end{aligned}
$$




$$
\begin{aligned}
& \leq C(M+L) T^{\theta / p^{\prime}}\|\varphi\|_{L_{p}\left((0, T), H_{q}^{2}(\Omega)\right)} \\
& \quad+\left\|\nabla\left(\frac{\rho_{1} \rho_{2}}{\mathfrak{p} \rho}-\frac{\rho_{10} \rho_{20}}{\mathfrak{p}_{0} \rho_{0}}\right)\right\|_{L_{\infty}\left((0, T), L_{q}(\Omega)\right)}\|\nabla \varphi\|_{L_{\infty}\left((0, T), L_{\infty}(\Omega)\right)} T^{1 / p} \\
& \leq C\left(M(M+L) T^{\theta / p^{\prime}}+(M+L)^{2} T^{1 / p}\right) .
\end{aligned}
$$

Next, we estimate nonlinear terms from the Lagrange transformation. In (4.4), we set $U=(\omega, \mathbf{w}, \varphi)$. Recall that $3<q<\infty$. By Sobolev's inequality and (5.6), we have

$$
\|\omega \operatorname{div} \mathbf{w}\|_{H_{q}^{1}(\Omega)} \leq C\|\omega\|_{H_{q}^{1}(\Omega)}\|\mathbf{w}\|_{H_{q}^{2}(\Omega)} \leq C T^{1 / p^{\prime}} M\|\mathbf{w}\|_{H_{q}^{2}(\Omega)},
$$

and so, we have

$$
\|\omega \operatorname{div} \mathbf{w}\|_{L_{p}\left((0, T), H_{q}^{1}(\Omega)\right)} \leq C T^{1 / p^{\prime}} M\|\mathbf{w}\|_{L_{p}\left((0, T), H_{q}^{2}(\Omega)\right)} \leq C T^{1 / p^{\prime}} M^{2} .
$$

Replacing $\mathbf{v}$ by $\mathbf{w}$ in (3.2), by Sobolev's inequality and (5.6), we have

$$
\begin{aligned}
\left\|R_{1}\right\|_{H_{q}^{1}(\Omega)} & \leq C\left(\left\|\rho_{0}\right\|_{H_{q}^{1}(\Omega)}+\|\omega\|_{H_{q}^{1}(\Omega)}\right) \int_{0}^{t}\|\mathbf{w}(\cdot, s)\|_{H_{q}^{2}(\Omega)} d s\|\mathbf{w}(\cdot, t)\|_{H_{q}^{2}(\Omega)} \\
& \leq C(L+M) T^{1 / p^{\prime}}\|\mathbf{w}\|_{L_{p}\left((0, T), H_{q}^{2}(\Omega)\right.}\|\mathbf{w}(\cdot, t)\|_{H_{q}^{2}(\Omega)},
\end{aligned}
$$

and so, we have

$$
\left\|R_{1}\right\|_{L_{p}\left((0, T), H_{q}^{1}(\Omega)\right)} \leq C(L+M) M^{2} T^{1 / p^{\prime}}
$$

Thus, we obtain

$$
\left\|f_{1}(U)\right\|_{L_{p}\left((0, T), H_{q}^{1}(\Omega)\right)} \leq C\left(M^{2}+(L+M) M^{2}\right) T^{1 / p^{\prime}} .
$$

Next, we consider $\mathbf{f}_{2}(U)$. By (5.11), we have

$$
\begin{aligned}
\left\|\int_{0}^{t} \nabla \mathbf{w}(\cdot, s) d s \nabla^{2} \mathbf{w}(\cdot, t)\right\|_{L_{q}(\Omega)} & \leq T\|\nabla \mathbf{w}\|_{L_{\infty}(0, T), L_{\infty}(\Omega)}\left\|\nabla^{2} \mathbf{w}(\cdot, t)\right\|_{L_{q}(\Omega)} \\
& \leq C M T\left\|\nabla^{2} \mathbf{w}(\cdot, t)\right\|_{L_{q}(\Omega)},
\end{aligned}
$$

and therefore

$$
\left\|\int_{0}^{t} \nabla \mathbf{w}(\cdot, s) d s \nabla^{2} \mathbf{w}(\cdot, t)\right\|_{L_{p}\left((0, T), L_{q}(\Omega)\right)} \leq C T M L .
$$

By Hölder's inequality and (5.11), we also get

$$
\begin{aligned}
\left\|\int_{0}^{t} \nabla^{2} \mathbf{w}(\cdot, s) d s \nabla \mathbf{w}(\cdot, t)\right\|_{L_{q}(\Omega)} & \leq T^{1 / p^{\prime}}\left(\int_{0}^{T}\left\|\nabla^{2} \mathbf{w}(\cdot, t)\right\|_{L_{q}(\Omega)}^{p}\right)^{1 / p}\|\nabla \mathbf{w}(\cdot, t)\|_{L_{\infty}(\Omega)} \\
& \leq C M T^{1 / p^{\prime}}\|\mathbf{w}\|_{L_{p}\left((0, T), H_{q}^{2}(\Omega)\right)} \leq C T M L .
\end{aligned}
$$

In this way, setting $\mathbf{k}_{\mathbf{w}}=\int_{0}^{t} \nabla \mathbf{w} d s$, we have

$$
\left\|\left(A_{2 \Delta}\left(\mathbf{k}_{\mathbf{w}}\right) \nabla^{2} \mathbf{w}, A_{1 \Delta}\left(\mathbf{k}_{\mathbf{w}}\right) \nabla \mathbf{w}, A_{2 \operatorname{div}}\left(\mathbf{k}_{\mathbf{w}}\right) \nabla^{2} \mathbf{w}, A_{1 \operatorname{div}}\left(\mathbf{k}_{\mathbf{w}}\right) \nabla \mathbf{w}\right)\right\|_{L_{p}\left((0, T), L_{q}(\Omega)\right)} \leq C T L M .
$$

By (5.6), (5.13), (5.14), and Sobolev's inequality we obtain

$$
\begin{aligned}
\left\|\frac{\rho_{0}+\omega}{\Sigma_{\rho}} \mathbf{V}^{0}\left(\mathbf{k}_{\mathbf{w}}\right) \nabla\left(\rho_{0}+\omega\right)\right\|_{L_{q}(\Omega)} & \leq C \int_{0}^{T}\|\nabla \mathbf{w}(\cdot, s)\|_{H_{q}^{1}(\Omega)} d s\left(\left\|\nabla \rho_{0}\right\|_{L_{q}(\Omega)}+\|\nabla \omega(\cdot, t)\|_{L_{q}(\Omega)}\right. \\
& \leq C T^{1 / p^{\prime}}\|\mathbf{w}\|_{L_{p}\left((0, T), H_{q}^{2}(\Omega)\right)}\left(L+\|\nabla \omega(\cdot, t)\|_{L_{q}(\Omega)}\right) \leq C T(L+M) M .
\end{aligned}
$$

Analogously, (5.13), (5.14) and Sobolev's inequality give

$$
\left\|\frac{\left(m_{1}-m_{2}\right) \rho_{1} \rho_{2}}{\Sigma_{\rho}} \mathbf{V}^{0}\left(\mathbf{k}_{\mathbf{w}}\right) \nabla \varphi\right\|_{L_{q}(\Omega)} \leq C T^{1 / p^{\prime}}\|\mathbf{w}\|_{L_{p}\left((0, T), H_{q}^{2}(\Omega)\right)}\|\nabla \varphi(\cdot, t)\|_{L_{q}(\Omega)} \leq C T M^{2} .
$$


Putting the estimates above and the estimates obtained in (5.23) together gives

$$
\begin{aligned}
\left\|\mathbf{f}_{2}(U)\right\|_{L_{p}\left((0, T), L_{q}(\Omega)\right)} \leq & C\left\{\left(L M+M^{2}+L^{2}\right) T+(M+L)^{3} T^{\left(\theta / p^{\prime}+1 / p\right)}\right. \\
& \left.+(M+L) T^{1 / p}+L^{2} T^{1 / p}+(M+L) L T^{\left(\theta / p^{\prime}+1 / p\right)}\right\} .
\end{aligned}
$$

Next, we consider $R_{3}$ defined in (3.4) replacing $\vartheta$ and $\mathbf{v}$ by $\varphi$ and $\mathbf{w}$. By (5.22), (5.11), Sobolev's inequality and by Hölder's inequality

$$
\begin{array}{r}
\left\|\nabla\left(\frac{\rho_{1} \rho_{2}}{\mathfrak{p} \rho}\right)\left(2 \mathbf{V}^{0}\left(\mathbf{k}_{\mathbf{w}}\right)+\left(\mathbf{V}^{0}\left(\mathbf{k}_{\mathbf{w}}\right)\right)^{2}\right) \nabla \varphi\right\|_{L_{q}(\Omega)} \leq C(M+L) \int_{0}^{T}\|\mathbf{w}(\cdot, s)\|_{H_{q}^{2}(\Omega)} \\
d s\|\nabla \varphi(\cdot, t)\|_{L_{q}(\Omega)} \\
\leq C(M+L) M^{2} T
\end{array}
$$

Other terms in $R_{3}$ can be estimated in the similar manner to the estimate of $R_{2}$, hence we obtain

$$
\left\|R_{3}\right\|_{L_{p}\left((0, T), L_{q}(\Omega)\right)} \leq C(M+L) L T
$$

which, combined with the estimates obtained in (5.23), leads to

$$
\begin{aligned}
\left\|f_{3}(U)\right\|_{L_{p}\left((0, T), L_{q}(\Omega)\right) \leq} & C\left(\left(L M+M^{2}+L^{2}\right) T+M(M+L) T^{\theta / p^{\prime}}\right. \\
& \left.+(M+L)^{2} T^{\left(\theta / p^{\prime}+1 / p\right)}+M(M+L) T^{\theta / p^{\prime}}+(M+L)^{2} T^{1 / p}\right) .
\end{aligned}
$$

Finally, we estimate $R_{4}$ defined in (3.5) replacing $\mathbf{v}$ and $\vartheta$ by $\mathbf{w}$ and $\varphi$. For this purpose, we have to extend $R_{4}$ to the whole time interval $\mathbb{R}$. Let $e_{T}$ be the extension operator defined in (5.7). Let $\tilde{h}_{0}$ be a function in $B_{q, p}^{2(1-1 / p)}\left(\mathbb{R}^{N}\right)$ such that $\tilde{h}_{0}=h_{0}$ in $\Omega$ and

$$
\left\|\tilde{h}_{0}\right\|_{B_{q, p}^{2(1-1 / p)}\left(\mathbb{R}^{N}\right)} \leq C\left\|h_{0}\right\|_{B_{q, p}^{2(1-1 / p)}(\Omega)} .
$$

Let

$$
T(t) h_{0}=e^{(\Delta-2) t} \tilde{h}_{0}=\mathcal{F}^{-1}\left[e^{-\left(|\xi|^{2}+2\right) t} \mathcal{F}\left[\tilde{h}_{0}\right](\xi)\right]
$$

where $\mathcal{F}$ and $\mathcal{F}^{-1}$ denote the Fourier transform on $\mathbb{R}^{N}$ and its inverse transform. We know that

$$
\left\|e^{t} T(\cdot) h\right\|_{L_{p}\left((0, \infty), H_{q}^{2}\left(\mathbb{R}^{N}\right)\right)}+\left\|e^{t} \partial_{t} T(\cdot) h\right\|_{L_{p}\left((0, \infty), L_{q}\left(\mathbb{R}^{N}\right)\right)} \leq C\|h\|_{B_{q, p}^{2(1-1 / p)}(\Omega)} .
$$

Let $\psi(t) \in C^{\infty}(\mathbb{R})$ be one for $t>-1$ and zero for $t<-2$. Since $\left.\omega\right|_{t=0}-\left.T(t) h\right|_{t=0}=h-h=0$ in $\Omega$, we set

$$
\tilde{e}_{T}[\omega]=e_{T}[\omega-T(\cdot) h]+\psi(t) T(|t|) h .
$$

Then, by (5.7), (5.8) and (5.27), we have

$$
\left\|e^{-\gamma t} \tilde{e}_{T}[\omega]\right\|_{L_{p}\left(\mathbb{R}, H_{q}^{2}(\Omega)\right)}+\left\|e^{-\gamma t} \partial_{t} \tilde{e}_{T}[\omega]\right\|_{L_{p}\left(\mathbb{R}, L_{q}(\Omega)\right)} \leq C\left(e^{2 \gamma} L+M\right)
$$

for any $\gamma \geq 0$, where $C$ is a constant independent of $\gamma, T, L$, and $M$. To treat $R_{4}$, setting

$$
\mathcal{R}_{\mathbf{w}}=-\left\{\mathbf{n}\left(y+\int_{0}^{t} \mathbf{w}(y, s) d s\right) \mathbf{V}^{0}\left(\mathbf{k}_{\mathbf{w}}\right)+\int_{0}^{1}(\nabla \mathbf{n})\left(y+\tau \int_{0}^{t} \mathbf{w}(y, s) d s\right) d \tau \int_{0}^{t} \mathbf{w}(y, s) d s\right\}
$$

we write it as $R_{4}=\mathcal{R}_{\mathbf{w}} \nabla \varphi$. Here, we may assume that $\mathbf{n}$ is defined in $\mathbb{R}^{N}$ and $\|\mathbf{n}\|_{H_{\infty}^{2}\left(\mathbb{R}^{N}\right)} \leq C$. Notice that $\left.\mathcal{R}_{\mathrm{w}}\right|_{t=0}=0$. We then define $\tilde{R}_{4}$ by

$$
\tilde{R}_{4}=e_{T}\left[\mathcal{R}_{\mathbf{w}}\right] \nabla\left(\tilde{e}_{T}[\varphi]\right)
$$

$\tilde{R}_{4}$ is an extension of $R_{4}$ to the whole time interval $\mathbb{R}$. Obviously, $\tilde{R}_{4}=R_{4}$ in $(0, T)$.

To estimate $\tilde{R}_{4}$, we use the following lemma due to Shibata and Shimizu [40]. 
Lemma 5. Let $1<p<\infty, 3<q<\infty$ and $0<T \leq 1$. Assume that $\Omega$ is a uniformly $C^{2}$ domain. Let

$$
f \in H_{\infty}^{1}\left(\mathbb{R}, L_{q}(\Omega)\right) \cap L_{\infty}\left(\mathbb{R}, H_{q}^{1}(\Omega)\right), \quad g \in L_{p}\left(\mathbb{R}, H_{q}^{1}(\Omega)\right) \cap H_{p}^{1 / 2}\left(\mathbb{R}, L_{q}(\Omega)\right) .
$$

If we assume that $f \in L_{p}\left(\mathbb{R}, H_{q}^{1}(\Omega)\right)$ and that $f$ vanishes for $t \in[0,2 T]$ in addition, then we have

$$
\begin{aligned}
& \|f g\|_{L_{p}\left(\mathbb{R}, H_{q}^{1}(\Omega)\right)}+\|f g\|_{H_{p}^{1 / 2}\left(\mathbb{R}, L_{q}(\Omega)\right)} \\
& \quad \leq C\left(\|f\|_{L_{\infty}\left(\mathbb{R}, H_{q}^{1}(\Omega)\right)}+T^{(q-3) /(p q)}\left\|\partial_{t} f\right\|_{L_{\infty}\left(\mathbb{R}, L_{q}(\Omega)\right)}^{(1-3 /(2 q))}\left\|\partial_{t} f\right\|_{L_{p}\left(\left(\mathbb{R}, H_{q}^{1}(\Omega)\right)\right.}^{3 /(2 q)}\right)\left(\|g\|_{p\left(\mathbb{R}, H_{q}^{1}(\Omega)\right)}+\|g\|_{H_{p}^{1 / 2}\left(\mathbb{R}, L_{q}(\Omega)\right)}\right) .
\end{aligned}
$$

Remark 6. (1) The boundary of $\Omega$ was assumed to be bounded in Shibata-Shimizu [40. But, Lemma 5 can be proved with the help of Sobolev's inequality and complex interpolation theorem, and so employing the same argument as that in the proof of [40, Lemma 2.7], we can prove Lemma 5 .

(2) By Sobolev's inequality, $\|f g\|_{H_{q}^{1}(\Omega)} \leq C\|f\|_{H_{q}^{1}(\Omega)}\|g\|_{L_{q}(\Omega)}$, and so the essential part of Lemma 5 is the estimate of $\|f g\|_{H_{p}^{1 / 2}\left(\mathbb{R}, L_{q}(\Omega)\right)}$.

Since $\Omega$ is a uniformly $C^{3}$ domain, we may assume that $\mathbf{n}$ is defined on the whole $\mathbb{R}^{N}$ and $\|\mathbf{n}\|_{H_{\infty}^{2}\left(\mathbb{R}^{N}\right)}<$ $\infty$. We then have

$$
\left\|e_{T}\left[\mathcal{R}_{\mathbf{w}}\right](\cdot, t)\right\|_{H_{q}^{1}(\Omega)} \leq C\left\{\int_{0}^{T}\|\mathbf{w}(\cdot, s)\|_{H_{q}^{2}(\Omega)} d s+\left(\int_{0}^{T}\|\mathbf{w}(\cdot, s)\|_{H_{q}^{1}(\Omega)} d s\right)^{2}\right\} \leq C\left(T^{1 / p^{\prime}} M+T^{2 / p^{\prime}} M^{2}\right)
$$

and so

$$
\left\|e_{T}\left[\mathcal{R}_{\mathbf{w}}\right]\right\|_{L_{\infty}\left(\mathbb{R}, H_{q}^{1}(\Omega)\right)} \leq C\left(T^{1 / p^{\prime}} M+T^{2 / p^{\prime}} M^{2}\right) .
$$

Choosing $T>$ so small that $T^{1 / p^{\prime}} M \leq 1$, by (5.8) we have

$$
\left\|\partial_{t} e_{T}\left[\mathcal{R}_{\mathbf{w}}\right](\cdot, t)\right\|_{H_{q}^{1}(\Omega)} \leq C \begin{cases}0 & \text { for } t<0, \\ \|\mathbf{w}(\cdot, t)\|_{H_{q}^{2}(\Omega)} & \text { for } 0<t<T, \\ \|\mathbf{w}(\cdot, 2 T-t)\|_{H_{q}^{2}(\Omega)} & \text { for } T<t<2 T \\ 0 & \text { for } t>2 T\end{cases}
$$

and therefore

$$
\left\|\partial_{t} e_{T}\left[\mathcal{R}_{\mathbf{w}}\right]\right\|_{L_{p}\left(\mathbb{R}, H_{q}^{1}(\Omega)\right)} \leq C\|\mathbf{w}\|_{L_{p}\left((0, T), H_{q}^{2}(\Omega)\right)} \leq C M .
$$

To estimate $\nabla\left(\tilde{e}_{T}[\varphi]\right)$, we use the following lemma.

Lemma 7. Let $1<p, q<\infty$. Assume that $\Omega$ is a uniform $C^{2}$ domain. Then

$$
H_{p}^{1}\left(\mathbb{R}, L_{q}(\Omega)\right) \cap L_{p}\left(\mathbb{R}, H_{q}^{2}(\Omega)\right) \subset H_{p}^{1 / 2}\left(\mathbb{R}, H_{q}^{1}(\Omega)\right),
$$

and

$$
\|\nabla u\|_{H_{p}^{1 / 2}\left(\mathbb{R}, L_{q}(\Omega)\right)} \leq C\left(\|u\|_{L_{p}\left(\mathbb{R}, H_{q}^{2}(\Omega)\right)}+\left\|\partial_{t} u\right\|_{L_{p}\left(\mathbb{R}, L_{q}(\Omega)\right)}\right)
$$

Remark 8. As was mentioned in Shibata and Shimizu [41, in the case that $\Omega=\mathbb{R}^{N}$, Lemma 7 can be proved by Weis's operator valued Fourier multiplier theorem. In the uniformly $C^{2}$ domain case, localizing the estimate, using the uniformity of the domain and the partition of unity, we can prove Lemma 7 . The detailed proof was given in Shibata [39]. In the case that $p=q$ and $\Omega$ is bounded, Lemma 7 was proved by M. Meyries and R. Schnaubelt [29].

Applying Lemma 7 and using (5.27), we have

$$
\begin{aligned}
& \left\|e^{-\gamma t} \nabla \tilde{e}_{T}[\varphi]\right\|_{H_{p}^{1 / 2}\left(\mathbb{R}, L_{q}(\Omega)\right)}+\left\|e^{-\gamma t} \nabla \tilde{e}_{T}[\varphi]\right\|_{L_{p}\left(\mathbb{R}, H_{q}^{1}(\Omega)\right)} \\
& \quad \leq C\left(\left\|e^{-\gamma t} \tilde{e}_{T}[\varphi]\right\|_{H_{p}^{1}\left(\mathbb{R}, L_{q}(\Omega)\right)}+\left\|e^{-\gamma t} \tilde{e}_{T}[\varphi]\right\|_{L_{p}\left(\mathbb{R}, H_{q}^{2}(\Omega)\right)}\right. \\
& \quad \leq C\left(\|\varphi\|_{L_{p}\left((0, T), H_{q}^{2}(\Omega)\right)}+\|\varphi\|_{H_{p}^{1}\left((0, T), L_{q}(\Omega)\right)}+e^{2 \gamma} L+M\right) \leq C\left(e^{2 \gamma} L+M\right)
\end{aligned}
$$


for any $\gamma>0$. Since $e_{T}\left[\mathcal{R}_{\mathbf{w}}\right]=0$ for $t \notin(0,2 T)$, applying Lemma 5 to $\tilde{R}_{4}$ and using two estimates (5.29), (5.30) and (5.31), we have

$$
\left\|e^{-\gamma t} \tilde{R}_{4}\right\|_{L_{p}\left(\mathbb{R}, H_{q}^{1}(\Omega)\right)}+\left\|e^{-\gamma t} \tilde{R}_{4}\right\|_{H_{p}^{1 / 2}\left(\mathbb{R}, L_{q}(\Omega)\right)} \leq C\left(T^{1 / p^{\prime}} M+T^{(q-3) /(p q)} M\right)\left(e^{2 \gamma} L+M\right)
$$

for any $\gamma>0$.

Applying Theorem 3 to Eq. (4.4), using (5.24), (5.25), (5.26), and (5.32), noting that $0<T \leq 1$, and fixing $\gamma>0$ a large positive number, we see that there exists three positive constants $C$ and $C_{M, L, \gamma}$ and $\tau$ for which

$$
[\zeta, \mathbf{v}, \vartheta]_{T} \leq C e^{2 \gamma T}\left(L+T^{\tau} C_{M, L, \gamma}\right) .
$$

Here, $C_{M, L, \gamma}$ is a constant depending on $L, M$, and $\gamma$. Letting $M=2 C e^{2 \gamma} L$ and choosing $T>0$ so small that $T^{\tau} C_{M, L, \gamma} \leq L$, we have

$$
[\zeta, \mathbf{v}, \vartheta]_{T} \leq M
$$

Let $\mathbf{S}$ be a map acting on $U=(\omega, \mathbf{w}, \varphi) \in \mathcal{H}_{T, M}$ defined by $\mathbf{S} U=V$, where $V=(\zeta, \mathbf{v}, \vartheta)$ is a unique solution of Eq. (5.1), and then by (5.34) we see that $\mathbf{S}$ maps $\mathcal{H}_{T, M}$ into itself. Let $U_{1}, U_{2} \in \mathcal{H}_{T, M}$, and then applying the same argument as that in the proof of (5.33) to $V_{1}-V_{2}$ with $V_{i}=\mathbf{S} U_{i}$, we see that there exists a constant $K$ depending on $M$ and $L$ for which

$$
\left[\mathbf{S} U_{1}-\mathbf{S} U_{2}\right]_{T} \leq K T^{\tau}\left[U_{1}-U_{2}\right]_{T} .
$$

Here, $\left.\left(U_{1}-U_{2}\right)\right|_{t=0}=0$, and so constructing the extension of the term corresponding to $R_{4}$ in the previous argument we can use $e_{T}\left[\varphi_{1}-\varphi_{2}\right]$ instead of $\tilde{e}_{T}\left[\varphi_{1}-\varphi_{2}\right]$. Namely, we de not need to use the operator $T(\cdot)$, and so $\gamma$ does not appear in the estimate, because $e_{T}\left[\varphi_{1}-\varphi_{2}\right]$ vanishes for $t \notin(0,2 T)$.

From (5.35) we see that $\mathbf{S}$ is a contraction map from $\mathcal{H}_{T, M}$ into itself, and so by the Banach fixed point theorem there exists a unique $V=(\zeta, \mathbf{v}, \vartheta) \in \mathcal{H}_{T, M}$ with $M=2 C L$ such that $V=\mathbf{S} V$. This $V$ is a unique solution of Eq. (4.4), which completes the proof of Theorem 1.

Employing the same argument as that in the proof of Theorem 1 we can prove the following theorem, which is so called almost global existence theorem and used to prove the global well-posedness.

Theorem 9. Let $2<p<\infty, 3<q<\infty$ and $T>0$. Assume that $2 / p+3 / q<1$ and that $\Omega$ is a uniform $C^{3}$ domain in $\mathbb{R}^{N}(N \geq 2)$. Let $\rho_{10}(x), \rho_{20}(x)$, and $\mathbf{u}_{0}(x)$ be initial data for Eq. (1.1). Assume that there exist positive numbers $a_{1}$ and $a_{2}$ for which

$$
a_{1} \leq \rho_{10}(x), \quad \rho_{20}(x) \leq a_{2} \quad \text { for any } x \in \bar{\Omega} .
$$

Let $\left(h_{0}(x), \rho_{0}(x)\right)=\Psi\left(\rho_{10}(x), \rho_{20}(x)\right)$. Then, there exists a small constant $\epsilon_{0}>0$ depending on $a_{1}, a_{2}$ and $T$ such that if $\rho_{10}, \rho_{20}, \mathbf{u}_{0}$ and $h_{0}$ satisfy the condition:

$$
\left\|\nabla\left(\rho_{10}, \rho_{20}\right)\right\|_{L_{q}(\Omega)}+\left\|\mathbf{u}_{0}\right\|_{B_{q, p}^{2(1-1 / p)}(\Omega)}+\left\|h_{0}\right\|_{B_{q, p}^{2(1-1 / p)}(\Omega)} \leq \epsilon_{0}
$$

and the compatibility condition:

$$
\left.\mathbf{u}_{0}\right|_{\Gamma}=0,\left.\quad\left(\nabla h_{0}\right) \cdot \mathbf{n}\right|_{\Gamma}=0,
$$

then problem (2.15) admits a unique solution $(\eta, \mathbf{v}, \vartheta)$ with

$$
\begin{gathered}
\eta-\rho_{0} \in H_{p}^{1}\left((0, T), H_{q}^{1}(\Omega)\right), \quad \mathbf{v} \in H_{p}^{1}\left((0, T), L_{q}(\Omega)^{3}\right) \cap L_{p}\left((0, T), H_{q}^{2}(\Omega)^{3}\right), \\
\vartheta \in H_{p}^{1}\left((0, T), L_{q}(\Omega)\right) \cap L_{p}\left((0, T), H_{q}^{2}(\Omega)\right)
\end{gathered}
$$

possessing the estimates:

$$
\begin{gathered}
\left\|\eta-\rho_{0}\right\|_{H_{p}^{1}\left((0, T), H_{q}^{1}(\Omega)\right)}+\left\|\partial_{t}(\mathbf{v}, \vartheta)\right\|_{L_{p}\left((0, T), L_{q}(\Omega)\right)}+\|(\mathbf{v}, \vartheta)\|_{L_{p}\left((0, T), H_{q}^{2}(\Omega)\right)} \leq C \epsilon_{0}, \\
a_{1} \leq \rho(x, t) \leq 2 a_{2}+a_{1} \quad \text { for }(x, t) \in \Omega \times(0, T), \quad \int_{0}^{T}\|\nabla \mathbf{v}(\cdot, s)\|_{L_{\infty}(\Omega)} \leq \delta .
\end{gathered}
$$

Here, $C$ is some constant independent of $\epsilon_{0}$. 


\section{Global well-posedness - proof of Theorem 2}

In this section, $\Omega$ is a bounded domain whose boundary $\Gamma$ is a compact hypersurface of $C^{3}$ class. Let $\rho_{1 *}$ and $\rho_{2 *}$ be any positive numbers and set $\left(h_{*}, \rho_{*}\right)=\Psi\left(\rho_{1 *}, \rho_{2 *}\right) \in \mathbb{R} \times \mathbb{R}_{+}$. Let $T>0$ and let $(\eta, \mathbf{v}, \vartheta)$ be a solution of Eq. (2.15) such that

$$
\begin{gathered}
\eta \in H_{p}^{1}\left((0, T), H_{q}^{1}(\Omega)\right), \quad \mathbf{v} \in H_{p}^{1}\left((0, T), L_{q}(\Omega)^{3}\right) \cap L_{p}\left((0, T), H_{q}^{2}(\Omega)^{3}\right), \\
\vartheta \in H_{p}^{1}\left((0, T), L_{q}(\Omega)\right) \cap L_{p}\left((0, T), H_{q}^{2}(\Omega)\right), \quad \int_{0}^{T}\|\nabla \mathbf{v}(\cdot, s)\|_{L_{\infty}(\Omega)} d s \leq \delta \\
\rho_{*} / 4 \leq \eta(x, t) \leq 4 \rho_{*}, \quad|\vartheta(x, t)| \leq 4\left|h_{*}\right| \quad \text { for }(x, t) \in \Omega \times(0, T) .
\end{gathered}
$$

To prove the global well-posedness, we prolong $(\eta, \mathbf{v}, \vartheta)$ to any time interval beyond $T$. Let $\zeta=\eta-\rho_{*}$ and $h=\vartheta-h_{*}$, and let

$$
\begin{aligned}
\mathcal{I}= & \left\|\rho_{0}-\rho_{*}\right\|_{H_{q}^{1}(\Omega)}+\left\|\left(\mathbf{u}_{0}, h_{0}-h_{*}\right)\right\|_{B_{q, p}^{2(1-1 / p)}}, \\
<e^{\gamma t} V>_{T}= & \left\|e^{\gamma t} \nabla \zeta\right\|_{L_{p}\left((0, T), L_{q}(\Omega)\right)}+\left\|e^{\gamma t} \partial_{t} \zeta\right\|_{L_{p}\left((0, T), H_{q}^{1}(\Omega)\right)}+\left\|e^{\gamma t} \mathbf{v}\right\|_{L_{p}\left((0, T), H_{q}^{2}(\Omega)\right.} \\
& +\left\|e^{\gamma t} \nabla h\right\|_{L_{p}\left((0, T), H_{q}^{1}(\Omega)\right)}+\left\|e^{\gamma t} \partial_{t}(\mathbf{v}, h)\right\|_{L_{p}\left((0, T), L_{q}(\Omega)\right)} .
\end{aligned}
$$

Here, $\gamma$ is a positive constant appearing in Theorem 10 below. The key step is to prove the estimate:

$$
<e^{\gamma t} V>_{T} \leq C\left(\mathcal{I}+<e^{\gamma t} V>_{T}^{2}\right)
$$

for some constant $C>0$.

To prove (6.2), we linearize Eq. (2.15) at $\left(\rho_{1}, \rho_{2}\right)=\left(\rho_{1 *}, \rho_{2 *}\right), \eta=\rho_{*}, \mathbf{v}=0$ and $\vartheta=h_{*}$. Namely, $\eta=\rho_{*}+\zeta, \mathbf{v}$ and $\vartheta=h_{*}+h$ satisfy the following equations:

$$
\left\{\begin{aligned}
\partial_{t} \zeta+a_{0 *} \operatorname{div} \mathbf{v}=\tilde{f}_{1}(U) & \text { in } \Omega \times(0, T), \\
a_{0 *} \partial_{t} \mathbf{v}-\mu \Delta \mathbf{v}-\nu \nabla \operatorname{div} \mathbf{v}+a_{1 *} \nabla \zeta+a_{2 *} \nabla h=\tilde{\mathbf{f}}_{2}(U) & \text { in } \Omega \times(0, T), \\
a_{3 *} \partial_{t} h+a_{2 *} \operatorname{div} \mathbf{v}-a_{4 *} \Delta h=\tilde{f}_{3}(U) & \text { in } \Omega \times(0, T), \\
\mathbf{v}=0, \quad(\nabla h) \cdot \mathbf{n}=g(U) & \text { on } \Gamma \times(0, T), \\
\left.(\zeta, \mathbf{v}, h)\right|_{t=0}=\left(\rho_{0}-\rho_{*}, \mathbf{u}_{0}, h_{0}-h_{*}\right) & \text { in } \Omega .
\end{aligned}\right.
$$

Here, we have set

$$
\begin{aligned}
a_{0 *}= & \rho_{*}, \quad a_{1 *}=\frac{a_{0}}{\Sigma_{\rho_{*}}}, \quad a_{2 *}=\frac{\left(m_{1}-m_{2}\right) \rho_{1 *} \rho_{2 *}}{\Sigma_{\rho_{*}}}, \quad a_{3 *}=\frac{m_{1} m_{2} \rho_{1 *} \rho_{2 *}}{\Sigma_{\rho_{*}}}, \quad a_{4 *}=\frac{\rho_{1 *} \rho_{2 *}}{\mathfrak{p}_{*} \rho_{*}}, \\
\Sigma_{\rho_{*}}= & m_{1} \rho_{1 *}+m_{2} \rho_{2 *}, \quad \mathfrak{p}_{*}=\frac{\rho_{1 *}}{m_{1}}+\frac{\rho_{2 *}}{m_{2}}, \quad U=(\eta, \mathbf{v}, \vartheta)=\left(\rho_{*}+\zeta, \mathbf{v}, h_{*}+h\right), \\
\tilde{f}_{1}(U)= & R_{1}(U)-\zeta \operatorname{div} \mathbf{v}, \\
\tilde{\mathbf{f}}_{2}(U)= & R_{2}(U)-\zeta \partial_{t} \mathbf{v}-\left(\frac{\eta}{\Sigma_{\rho}}-\frac{\rho_{*}}{\Sigma_{\rho_{*}}}\right) \nabla \zeta-\left(m_{1}-m_{2}\right)\left(\frac{\rho_{1} \rho_{2}}{\Sigma_{\rho}}-\frac{\rho_{1 *} \rho_{2 *}}{\Sigma_{\rho_{*}}}\right) \nabla h, \\
\tilde{f}_{3}(U)= & R_{3}(U)-m_{1} m_{2}\left(\frac{\rho_{1} \rho_{2}}{\Sigma_{\rho}}-\frac{\rho_{1 *} \rho_{2 *}}{\Sigma_{\rho_{*}}}\right) \nabla h-\left(m_{1}-m_{2}\right)\left(\frac{\rho_{1} \rho_{2}}{\Sigma_{\rho}}-\frac{\rho_{1 *} \rho_{2 *}}{\Sigma_{\rho_{*}}}\right) \operatorname{div} \mathbf{v} \\
& +\operatorname{div}\left(\left(\frac{\rho_{1} \rho_{2}}{\mathfrak{p} \rho}-\frac{\rho_{1 *} \rho_{2 *}}{\mathfrak{p}_{*} \rho_{*}}\right) \nabla h\right) \\
g(U)= & R_{4}(U) .
\end{aligned}
$$

Notice that $a_{0 *}, a_{1 *}, a_{3 *}$ and $a_{4 *}$ are positive constants, while $a_{2 *}$ is a real number. We consider the system of linear equations:

$$
\left\{\begin{aligned}
\partial_{t} \zeta+a_{0 *} \operatorname{div} \mathbf{v}=g_{1} & \text { in } \Omega \times(0, T), \\
a_{0 *} \partial_{t} \mathbf{v}-\mu \Delta \mathbf{v}-\nu \nabla \operatorname{div} \mathbf{v}+a_{1 *} \nabla \zeta+a_{2 *} \nabla \vartheta=\mathbf{g}_{2} & \text { in } \Omega \times(0, T), \\
a_{3 *} \partial_{t} \vartheta+a_{2 *} \operatorname{div} \mathbf{v}-a_{4 *} \Delta \vartheta=g_{3} & \text { in } \Omega \times(0, T), \\
\mathbf{v}=0, \quad(\nabla \vartheta) \cdot \mathbf{n}=g_{4} & \text { on } \Gamma \times(0, T), \\
\left.(\zeta, \mathbf{v}, \vartheta)\right|_{t=0}=\left(\zeta_{0}, \mathbf{v}_{0}, \vartheta_{0}\right) & \text { in } \Omega
\end{aligned}\right.
$$


For Eq. (6.4), we have the following decay theorem.

Theorem 10. Let $1<p, q<\infty, 2 / p+1 / q \neq 1$ and $2 / p+1 / q \neq 2$. Assume that $\Omega$ is a bounded domain whose boundary $\Gamma$ is a compact hypersurface of $C^{3}$ class. Let

$$
\begin{aligned}
& \rho_{0} \in H_{q}^{1}(\Omega), \quad \mathbf{v}_{0} \in B_{q, p}^{2(1-1 / p)}(\Omega)^{3}, \quad \vartheta_{0} \in B_{q, p}^{2(1-1 / p)}(\Omega), \\
& g_{1} \in L_{p}\left((0, T), H_{q}^{1}(\Omega)\right), \quad \mathbf{g}_{2} \in L_{p}\left((0, T), L_{q}(\Omega)^{3}\right) \cap H_{p}^{1}\left((0, T), L_{q}(\Omega)^{3}\right), \\
& g_{3} \in L_{p}\left((0, T), L_{q}(\Omega)\right), \quad E\left[e^{\gamma_{1} t} g_{4}\right] \in H_{p}^{1 / 2}\left(\mathbb{R}, L_{q}(\Omega)\right) \cap L_{p}\left(\mathbb{R}, H_{q}^{1}(\Omega)\right)
\end{aligned}
$$

for some $\gamma_{1}>0$. Here, $E\left[e^{\gamma_{1} t} g_{4}\right]$ denotes some extension of $e^{\gamma_{1} t} g_{4}$ to the whole time interval $\mathbb{R}$. Assume that $\mathbf{v}_{0}, \vartheta_{0}$ and $g_{4}$ satisfy the compatibility conditions:

$$
\mathbf{v}_{0}=0 \text { on } \Gamma \text { for } 2 / p+1 / q<2, \quad\left(\nabla \vartheta_{0}\right) \cdot \mathbf{n}=\left.g_{4}\right|_{t=0} \text { on } \Gamma \text { for } 2 / p+1 / q<1 .
$$

Then, problem (6.4) admits unique solutions $\eta, \mathbf{v}$, and $\vartheta$ with

$$
\begin{gathered}
\eta \in H_{p}^{1}\left((0, T), H_{q}^{1}(\Omega)\right), \quad \mathbf{v} \in L_{p}\left((0, T), H_{q}^{2}(\Omega)^{3}\right) \cap H_{p}^{1}\left((0, T), L_{q}(\Omega)^{3}\right), \\
\vartheta \in L_{p}\left((0, T), H_{q}^{2}(\Omega)\right) \cap H_{p}^{1}\left((0, T), L_{q}(\Omega)\right)
\end{gathered}
$$

possessing the estimate:

$$
\begin{aligned}
\left\|e^{\gamma t} \nabla \eta\right\|_{L_{p}\left((0, T), L_{q}(\Omega)\right)}+\left\|e^{\gamma t} \partial_{t} \eta\right\|_{L_{p}\left((0, T), H_{q}^{1}(\Omega)\right)}+\left\|e^{\gamma t} \mathbf{v}\right\|_{L_{p}\left((0, T), H_{q}^{2}(\Omega)\right.} \\
\quad+\left\|e^{\gamma t} \nabla \vartheta\right\|_{L_{p}\left((0, T), H_{q}^{1}(\Omega)\right)}+\left\|e^{\gamma t} \partial_{t}(\mathbf{v}, \vartheta)\right\|_{L_{p}\left((0, T), L_{q}(\Omega)\right)} \\
\leq C\left(\left\|\zeta_{0}\right\|_{H_{q}^{1}(\Omega)}+\left\|\left(\mathbf{v}_{0}, \vartheta_{0}\right)\right\|_{B_{q, p}^{2(1-1 / p)}(\Omega)}+\left\|e^{\gamma t}\left(g_{1}, \mathbf{g}_{2}, g_{3}\right)\right\|_{L_{p}\left((0, T), L_{q}(\Omega)\right)}\right. \\
\left.\quad+\left\|E\left[e^{\gamma_{1} t} g_{4}\right]\right\|_{H_{p}^{1 / 2}\left(\mathbb{R}, L_{q}(\Omega)\right)}+\left\|E\left[e^{\gamma_{1} t} g_{4}\right]\right\|_{L_{p}\left(\mathbb{R}, H_{q}^{1}(\Omega)\right)}\right)
\end{aligned}
$$

for some constants $\gamma \in\left(0, \gamma_{1}\right]$ and $C>0$.

Postponing the proof of Theorem 10, we prove (6.2). Let $\left(\rho_{1}(x), \rho_{2}(x)\right)=\Phi(\vartheta, \eta)$. Following the ideas from [37], we first prove that

$$
\begin{aligned}
\left\|\eta-\rho_{0}\right\|_{L_{\infty}\left((0, T), H_{q}^{1}(\Omega)\right)} & \leq C<e^{\gamma t} V>_{T}, \\
\left\|\vartheta-h_{0}\right\|_{L_{\infty}\left((0, T), B_{q, p}^{2(1-1 / p)}(\Omega)\right)} & \leq C\left(\mathcal{I}+<e^{\gamma t} V>_{T}\right) .
\end{aligned}
$$

where $\left(h_{0}, \rho_{0}\right)=\left.(\vartheta, \eta)\right|_{t=0}$ (cf. (2.15) in Introduction). In fact, by Hölder's inequality we have

$$
\begin{aligned}
\|\eta(\cdot, t)-\eta(\cdot, 0)\|_{H_{q}^{1}(\Omega)} & \leq \int_{0}^{T}\left\|\partial_{t} \eta(\cdot, t)\right\|_{H_{q}^{1}(\Omega)} d t \\
& \leq\left(\int_{0}^{T} e^{-p^{\prime} \gamma t} d t\right)^{1 / p^{\prime}}\left(\int_{0}^{T}\left(e^{\gamma t}\left\|\partial_{t} \eta(\cdot, t)\right\|_{H_{q}^{1}(\Omega)}\right)^{p} d t\right)^{1 / p} \leq C<e^{\gamma t} V>_{T} .
\end{aligned}
$$

Recalling that $\vartheta-h_{*}=h$ and $\vartheta_{0}-h_{*}=h_{0}-h_{*}$, we have

$$
\left\|\vartheta(\cdot, t)-\vartheta_{0}\right\|_{L_{q}(\Omega)} \leq \int_{0}^{T}\left\|\partial_{s} h(\cdot, s)\right\|_{L_{q}(\Omega)} d s+\left\|h_{0}-h_{*}\right\|_{L_{q}(\Omega)} \leq C\left(<e^{\gamma t} V>_{T}+\mathcal{I}\right) .
$$

Let $H(x, t)=h(x, t)-|\Omega|^{-1} \int_{\Omega} h(x, t) d x$. Since $\int_{\Omega} H(x, t) d x=0$, by Poincaré's inequality we have

$$
\|H(\cdot, t)\|_{H_{q}^{2}(\Omega)} \leq C\|\nabla H(\cdot, t)\|_{H_{q}^{1}(\Omega)}=C\|\nabla h(\cdot, t)\|_{H_{q}^{1}(\Omega)} .
$$

Moreover, noting that $2(1-1 / p)>1$, we have

$$
\begin{aligned}
\left\|\left.H\right|_{t=0}\right\|_{B_{q, p}^{2(1-1 / p)}(\Omega)} & \leq\left\|\left.H\right|_{t=0}\right\|_{L_{q}(\Omega)}+\left\|\left.\nabla H\right|_{t=0}\right\|_{B_{q, p}^{1-2 / p}(\Omega)} \leq C\left(\left\|h_{0}-h_{*}\right\|_{L_{q}(\Omega)}+\left\|\nabla h_{0}\right\|_{B_{q, p}^{1-2 / p}(\Omega)}\right) \\
& =C\left\|h_{0}-h_{*}\right\|_{B_{q, p}^{2(1-1 / p)}(\Omega)} .
\end{aligned}
$$


On the other hand, employing the same argument as that in the proof of (5.6), by real interpolation theory, we have

$$
\begin{aligned}
\sup _{t \in(0, T)}\|H(\cdot, t)\|_{B_{q, p}^{2(1-1 / p)}(\Omega)} & \leq \sup _{t \in(0, T)}\left\|\tilde{e}_{T}[H](\cdot, t)\right\|_{B_{q, p}^{2(1-1 / p)}(\Omega)} \\
& \leq C\left(\|H\|_{L_{p}\left((0, T), H_{q}^{2}(\Omega)\right)}+\left\|\partial_{t} H\right\|_{L_{p}\left((0, T), L_{q}(\Omega)\right)}+\left\|\left.H\right|_{t=0}\right\|_{B_{q, p}^{2(1-1 / p)}(\Omega)}\right) .
\end{aligned}
$$

Therefore, since $\left\|\partial_{t} H\right\|_{L_{q}(\Omega)} \leq C\left\|\partial_{t} h\right\|_{L_{q}(\Omega)}$, we obtain

$$
\sup _{t \in(0, T)}\|H(\cdot, t)\|_{B_{q, p}^{2(1-1 / p)}(\Omega)} \leq C\left(\|\nabla h\|_{L_{p}\left((0, T), H_{q}^{1}(\Omega)\right)}+\left\|\partial_{t} h\right\|_{L_{p}\left((0, T), L_{q}(\Omega)\right)}+\left\|h_{0}-h_{*}\right\|_{B_{q, p}^{2(1-1 / p)}(\Omega)}\right) .
$$

Since

$$
\sup _{t \in(0, T)}\|h(\cdot, t)\|_{L_{q}(\Omega)} \leq\left\|h_{0}-h_{*}\right\|_{L_{q}(\Omega)}+\int_{0}^{T}\left\|\partial_{t} h(\cdot, t)\right\|_{L_{q}(\Omega)} d t \leq C\left(\left\|h_{0}-h_{*}\right\|_{L_{q}(\Omega)}+C<e^{\gamma t} V>_{T}\right)
$$

we have

$$
\begin{aligned}
\sup _{t \in(0, T)}\|h\|_{B_{q, p}^{2(1-1 / p)}(\Omega)} & \leq \sup _{t \in(0, T)}\|H\|_{B_{q, p}^{2(1-1 / p)}(\Omega)}+\sup _{t \in(0, T)}\|h(\cdot, t)\|_{L_{q}(\Omega)} \\
& \leq C\left(\left\|h_{0}-h_{*}\right\|_{B_{q, p}^{2(1-1 / p)}(\Omega)}+<e^{\gamma t} V>_{T}\right) \leq C\left(\mathcal{I}+<e^{\gamma t} V>_{T}\right),
\end{aligned}
$$

which shows the second inequality in (6.5). Next we show that

$$
\left\|\left(\rho_{1}, \rho_{2}\right)-\left(\rho_{1 *}, \rho_{2 *}\right)\right\|_{L_{\infty}\left((0, T), H_{q}^{1}(\Omega)\right)} \leq C\left(\mathcal{I}+<e^{\gamma t} V>_{T}\right) .
$$

In fact, by the Taylor formula, we have

$$
\left(\rho_{1}, \rho_{2}\right)-\left(\rho_{10}, \rho_{20}\right)=\Phi(\vartheta, \eta)-\Phi\left(h_{0}, \rho_{0}\right) \leq \int_{0}^{1} \Phi^{\prime}\left(\left(h_{0}, \rho_{0}\right)+\theta\left(\vartheta-\vartheta_{0}, \eta-\eta_{0}\right)\right) d \theta\left(\vartheta-h_{0}, \eta-\rho_{0}\right)
$$

where $\left(h_{0}, \rho_{0}\right)=\left.(\vartheta, \eta)\right|_{t=0}$. Set

$$
D=\left\{(\zeta, \eta) \in \mathbb{R}^{2}|| \zeta|\leq| h_{*} \mid / 4, \quad \rho_{*} / 4 \leq \eta \leq 4 \rho_{*}\right\},
$$

and then by (6.1),$(\vartheta, \eta) \in D$ for any $(x, t) \in \Omega \times(0, T)$. Let $C_{0}$ be a positive constant for which

$$
\sup _{(\vartheta, \eta) \in D}\left|\Phi^{\prime}(\vartheta, \eta)\right| \leq C_{0}, \quad \sup _{(\vartheta, \eta) \in D}\left|\Phi^{\prime \prime}(\vartheta, \eta)(\vartheta, \eta)\right| \leq C_{0} .
$$

We then have

$$
\left\|\left(\rho_{1}, \rho_{2}\right)-\left(\rho_{10}, \rho_{20}\right)\right\|_{L_{\infty}\left((0, T), H_{q}^{1}(\Omega)\right)} \leq 3 C_{0}\left\|\left(\vartheta-h_{0}, \eta-\rho_{0}\right)\right\|_{L_{\infty}\left((0, T), H_{q}^{1}(\Omega)\right)},
$$

which, combined with (6.5), leads to (6.6), because $\left\|\left(\rho_{10}, \rho_{20}\right)-\left(\rho_{1 *}, \rho_{2 *}\right)\right\|_{H_{q}^{1}(\Omega)} \leq \mathcal{I}$.

By (6.1) we may assume that there exist two positive constants $a_{1}$ and $a_{2}$ depending on $\rho_{*}$ and $h_{*}$ for which

$$
a_{1} \leq \rho_{1}(x, t), \rho_{2}(x, t) \leq a_{2} \text { for any }(x, t) \in \Omega \times(0, T) .
$$

By (6.6) and (6.7), we have the following estimates:

$$
\begin{aligned}
& \left\|e^{\gamma t}\left(\frac{\eta}{\Sigma_{\rho}}-\frac{\rho_{*}}{\Sigma_{\rho_{*}}}\right) \nabla \zeta\right\|_{L_{\infty}\left((0, T), L_{q}(\Omega)\right)}+\left\|e^{\gamma t}\left(\frac{\rho_{1} \rho_{2}}{\Sigma_{\rho}}-\frac{\rho_{1 *} \rho_{2 *}}{\Sigma_{\rho_{*}}}\right) \nabla h\right\|_{L_{\infty}\left((0, T), L_{q}(\Omega)\right)} \\
& +\left\|e^{\gamma t} \operatorname{div}\left(\left(\frac{\rho_{1} \rho_{2}}{\mathfrak{p} \rho}-\frac{\rho_{1 *} \rho_{2 *}}{\mathfrak{p}_{*} \rho_{*}}\right) \nabla h\right)\right\|_{L_{\infty}\left((0, T), L_{q}(\Omega)\right)} \leq C\left(\mathcal{I}+<e^{\gamma t} V>_{T}\right)<e^{\gamma t} V>_{T} .
\end{aligned}
$$

By Sobolev's inequality and (6.5), we have

$$
\|\zeta\|_{H_{q}^{1}(\Omega)} \leq C\left\|\eta-\rho_{0}\right\|_{H_{q}^{1}(\Omega)}+\left\|\rho_{0}-\rho_{*}\right\|_{H_{q}^{1}(\Omega)} \leq C\left(\mathcal{I}+<e^{\gamma t} V>_{T}\right),
$$


and so

$$
\begin{aligned}
\left\|e^{\gamma t} \zeta \operatorname{div} \mathbf{v}\right\|_{L_{p}\left((0, T), H_{q}^{1}(\Omega)\right)} & \leq C\|\zeta\|_{L_{\infty}\left((0, T), H_{q}^{1}(\Omega)\right)}\left\|e^{\gamma t} \nabla \mathbf{v}\right\|_{L_{p}\left((0, T), H_{q}^{1}(\Omega)\right)} \\
& \leq C\left(\mathcal{I}+<e^{\gamma t} V>_{T}\right)<e^{\gamma t} V>_{T}
\end{aligned}
$$

By (5.27) and real interpolation theory, we have

$$
\|\mathbf{v}\|_{L_{\infty}\left((0, T), B_{q, p}^{2(1-1 / p)}(\Omega)\right)}+\|\mathbf{v}\|_{L_{\infty}\left((0, T), H_{\infty}^{1}(\Omega)\right)} \leq C\left(\mathcal{I}+<e^{\gamma t} V>_{T}\right) .
$$

In fact,

$$
\begin{aligned}
& \sup _{t \in(0, T)}\|\mathbf{v}(\cdot, t)\|_{B_{q, p}^{2(1-1 / p)}(\Omega)} \leq \sup _{t \in(0, T)}\left\|\tilde{e}_{T}[\mathbf{v}](\cdot, t)\right\|_{B_{q, p}^{2(1-1 / p)}(\Omega)} \\
& \leq C\left(\|\mathbf{v}\|_{L_{p}\left((0, T), H_{q}^{2}(\Omega)\right)}+\left\|\partial_{t} \mathbf{v}\right\|_{L_{p}\left((0, T), L_{q}(\Omega)\right)}+\left\|T(\cdot) \tilde{\mathbf{v}}_{0}\right\|_{L_{p}\left((0, \infty), H_{q}^{2}(\Omega)\right)}+\left\|\partial_{t} T(\cdot) \tilde{\mathbf{v}}_{0}\right\|_{L_{p}\left((0, \infty), L_{q}(\Omega)\right)}\right),
\end{aligned}
$$

where $\tilde{\mathbf{v}}_{0} \in B_{q, p}^{2(1-1 / p)}\left(\mathbb{R}^{3}\right)$ equals to $\mathbf{v}_{0}$ in $\Omega$ and $\left\|\tilde{\mathbf{v}}_{0}\right\|_{B_{q, p}^{2(1-1 / p)}\left(\mathbb{R}^{3}\right)} \leq C\left\|\mathbf{v}_{0}\right\|_{B_{q, p}^{2(1-1 / p)}(\Omega)}$. Thus, by (5.27), we have the estimate of the first term in (6.9). Since $2 / p+3 / q<1$, we have $\|\mathbf{v}\|_{H_{\infty}^{1}(\Omega)} \leq C\|\mathbf{v}\|_{B_{q, p}^{2(1-1 / p)}(\Omega)}$, which completes the proof of (6.9).

Now and we shall estimate $R_{i}(U)$. By Sobolev's inequality and Hölder's inequality, we have

$$
\begin{aligned}
& \left\|\int_{0}^{t} \nabla \mathbf{v}(\cdot, s) d s \nabla^{2} f\right\|_{L_{q}(\Omega)} \leq\left(\int_{0}^{T} e^{-\gamma p^{\prime} s} d s\right)^{1 / p^{\prime}}\left(\int_{0}^{T}\left(e^{\gamma s}\|\nabla \mathbf{v}(\cdot, s)\|_{L_{\infty}(\Omega)}\right)^{p} d s\right)^{1 / p}\left\|\nabla^{2} f(\cdot, t)\right\|_{L_{q}(\Omega)} \\
& \quad \leq C<e^{\gamma t} V>_{T}\|f(\cdot, t)\|_{H_{q}^{2}(\Omega)},
\end{aligned}
$$

and therefore

$$
\left\|e^{\gamma t} \int_{0}^{t} \nabla \mathbf{v}(\cdot, s) d s \nabla^{2} f\right\|_{L_{p}\left((0, T), L_{q}(\Omega)\right)} \leq C<e^{\gamma t} V>_{T}\left\|e^{\gamma t} f\right\|_{L_{p}\left((0, T), H_{q}^{2}(\Omega)\right)} .
$$

A similar estimate of $\left\|\int_{0}^{t} \nabla^{2} \mathbf{v}(\cdot, s) d s \nabla f\right\|_{L_{q}(\Omega)}$ yields

$$
\left\|e^{\gamma t} \int_{0}^{t} \nabla^{2} \mathbf{v}(\cdot, s) d s \nabla f\right\|_{L_{p}\left((0, T), L_{q}(\Omega)\right)} \leq C<e^{\gamma t} V>_{T}\left\|e^{\gamma t} f\right\|_{L_{p}\left((0, T), H_{q}^{2}(\Omega)\right)} .
$$

By (6.1) and (6.10), we have

$$
\begin{aligned}
\left\|e^{\gamma t} R_{1}(U)\right\|_{L_{p}\left((0, T), L_{q}(\Omega)\right)} & \leq C\left\|\int_{0}^{t} \nabla \mathbf{v}(\cdot, s) d s \nabla \mathbf{v}\right\|_{L_{p}\left((0, T), L_{q}(\Omega)\right)} \\
& \leq C<e^{\gamma t} V>_{T}\|\nabla \mathbf{v}(\cdot, t)\|_{L_{p}\left((0, T), L_{q}(\Omega)\right)} \leq C<e^{\gamma t} V>_{T}^{2} .
\end{aligned}
$$

Noting that $\nabla \eta=\nabla \zeta$, we have

$$
\nabla R_{1}(U)=-\sum_{i, j=1}^{3}\left(\nabla \zeta V_{i j}^{0}\left(\mathbf{k}_{\mathbf{v}}\right) \frac{\partial v_{i}}{\partial x_{j}}+\eta\left(\nabla_{\mathbf{k}} V_{i j}^{0}\right)\left(\mathbf{k}_{\mathbf{v}}\right) \int_{0}^{t} \nabla^{2} \mathbf{v}(\cdot, s) d s \frac{\partial v_{i}}{\partial x_{j}}+\eta V_{i j}^{0}\left(\mathbf{k}_{\mathbf{v}}\right) \nabla \frac{\partial v_{i}}{\partial x_{j}}\right) .
$$

Noting that $\left|\mathbf{k}_{\mathbf{v}}\right| \leq \delta$, we have

$$
\begin{aligned}
& \left\|e^{\gamma t} \nabla R_{1}(U)\right\|_{L_{p}\left((0, T), L_{q}(\Omega)\right)} \\
& \quad \leq C\left(\|\nabla \zeta\|_{L_{\infty}\left((0, T), L_{q}(\Omega)\right)}\left\|e^{\gamma t} \nabla \mathbf{v}\right\|_{L_{p}\left((0, T), L_{q}(\Omega)\right)}+<e^{\gamma t} V>_{T}\left\|e^{\gamma t} \nabla \mathbf{v}\right\|_{L_{p}\left((0, T), H_{q}^{1}(\Omega)\right)}\right) .
\end{aligned}
$$

Since

by (6.5) we have

$$
\|\nabla \zeta\|_{L_{q}(\Omega)} \leq\left\|\nabla\left(\eta-\rho_{0}\right)\right\|_{L_{q}(\Omega)}+\left\|\nabla \rho_{0}\right\|_{L_{q}(\Omega)}
$$

$$
\|\nabla \zeta\|_{L_{\infty}\left((0, T), L_{q}(\Omega)\right)} \leq C\left(\mathcal{I}+<e^{\gamma t} V>_{T}\right),
$$


and so, (6.10) and (6.11) imply

$$
\left\|e^{\gamma t} \nabla R_{1}(U)\right\|_{L_{p}\left((0, T), L_{q}(\Omega)\right)} \leq C\left(\mathcal{I}+<e^{\gamma t} V>_{T}\right)<e^{\gamma t} V>_{T} .
$$

Summing up, we have obtained

$$
\left\|e^{\gamma t} R_{1}(U)\right\|_{L_{p}\left((0, T), H_{q}^{1}(\Omega)\right)} \leq C\left(\mathcal{I}+<e^{\gamma t} V>_{T}\right)<e^{\gamma t} V>_{T} .
$$

We next consider $R_{2}(U)$ given in (3.3). By (6.10) and (6.11), we have

$$
\left\|e^{\gamma t}\left(A_{2 \Delta}\left(\mathbf{k}_{\mathbf{v}}\right) \nabla^{2} \mathbf{v}, A_{1 D}\left(\mathbf{k}_{\mathbf{v}}\right) \nabla \mathbf{v}, A_{2 \text { div }}\left(\mathbf{k}_{\mathbf{v}}\right) \nabla^{2} \mathbf{v}, A_{1 \text { div }}\left(\mathbf{k}_{\mathbf{v}}\right) \nabla \mathbf{v}\right)\right\|_{L_{p}\left((0, T), L_{q}(\Omega)\right)} \leq C<e^{\gamma t} V>_{T}^{2} .
$$

By (6.1) and (6.10) we have

$$
\left\|e^{\gamma t}\left(\frac{\eta}{\Sigma_{\rho}} \mathbf{V}^{0}\left(\mathbf{k}_{\mathbf{v}}\right) \nabla \zeta, \frac{\rho_{1} \rho_{2}}{\Sigma_{\rho}} \mathbf{V}^{0}\left(\mathbf{k}_{\mathbf{v}}\right) \nabla h\right)\right\|_{L_{p}\left((0, T), L_{q}(\Omega)\right)} \leq C<e^{\gamma t} V>_{T}^{2} .
$$

Summing up, we have obtained

$$
\left\|e^{\gamma t} R_{2}(U)\right\|_{L_{p}\left((0, T), H_{q}^{1}(\Omega)\right)} \leq C\left(\mathcal{I}+<e^{\gamma t} V>_{T}\right)<e^{\gamma t} V>_{T} .
$$

We next consider $R_{3}(U)$ given in (3.4). By (6.1) and (6.6), we have

$$
\begin{gathered}
a_{1} \leq \rho_{1}(x, t), \rho_{2}(x, t) \leq a_{2} \quad \text { for }(x, t) \in \Omega \times(0, T), \\
\left\|\nabla \rho_{i}\right\|_{L_{\infty}\left((0, T), L_{q}(\Omega)\right)} \leq C\left(\mathcal{I}+<e^{\gamma t} V>_{T}\right) \quad(i=1,2),
\end{gathered}
$$

where $a_{1}$ and $a_{2}$ are some positive constants depending on $\rho_{1 *}$ and $\rho_{2 *}$, and therefore

$$
\left\|\nabla\left(\frac{\rho_{1} \rho_{2}}{\mathfrak{p} \rho}\right)\right\|_{L_{\infty}\left((0, T), L_{q}(\Omega)\right)} \leq C\left(\mathcal{I}+<e^{\gamma t} V>_{T}\right) .
$$

Thus, by (6.1), (6.10), and (6.11), we have

$$
\left\|e^{\gamma t} R_{3}(U)\right\|_{L_{p}\left((0, T), H_{q}^{1}(\Omega)\right)} \leq C\left(\mathcal{I}+<e^{\gamma t} V>_{T}\right)<e^{\gamma t} V>_{T} .
$$

Finally, we estimate $R_{4}(U)$ given in (3.5). Similarly to Sect. [5 we set

$$
\mathcal{R}_{\mathbf{v}}=-\left\{\mathbf{n}\left(y+\int_{0}^{t} \mathbf{v}(y, s) d s\right) \mathbf{V}^{0}\left(\mathbf{k}_{\mathbf{v}}\right)+\int_{0}^{1}(\nabla \mathbf{n})\left(y+\tau \int_{0}^{t} \mathbf{v}(y, s) d s\right) d \tau \int_{0}^{t} \mathbf{v}(y, s) d s\right\} .
$$

Let $H(x, t)=h(x, t)-|\Omega|^{-1} \int_{\Omega} h(x, t) d x$. Obviously, $\nabla H=\nabla h$. Moreover, by Poincaré's inequality, we have

$$
\begin{aligned}
\left\|e^{\gamma t} H\right\|_{L_{p}\left((0, T), H_{q}^{2}(\Omega)\right)} & +\left\|e^{\gamma t} \partial_{t} H\right\|_{L_{p}\left((0, T), L_{q}(\Omega)\right)} \\
& \leq C\left(\left\|e^{\gamma t} \nabla h\right\|_{L_{p}\left((0, T), H_{q}^{1}(\Omega)\right)}+\left\|e^{\gamma t} \partial_{t} h\right\|_{L_{p}\left((0, T), L_{q}(\Omega)\right)}\right) .
\end{aligned}
$$

In particular, we can write $R_{4}(U)$ as $R_{4}(U)=\mathcal{R}_{\mathbf{v}} \nabla H$. We define the extension of $e^{\gamma t} R_{4}(U)$ by

$$
E\left[e^{\gamma t} R_{4}(U)\right]=e_{T}\left[\mathcal{R}_{\mathbf{v}}\right]\left(\nabla \tilde{e}_{T}\left[e^{\gamma t} H\right]\right) .
$$

To estimate $E\left[e^{\gamma t} R_{4}(U)\right]$, we use the following lemma.

Lemma 11. Let $1<p<\infty$ and $3<q<\infty$. Then, the following two assertions hold.

(1) If $f \in H_{\infty}^{1}\left(\mathbb{R}, L_{\infty}(\Omega)\right)$ and $g \in H_{p}^{1 / 2}\left(\mathbb{R}, L_{q}(\Omega)\right)$, then

$$
\|f g\|_{H_{p}^{1 / 2}\left(\mathbb{R}, L_{q}(\Omega)\right)} \leq C\|f\|_{H_{\infty}^{1}\left(\mathbb{R}, L_{\infty}(\Omega)\right)}\|g\|_{H_{p}^{1 / 2}\left(\mathbb{R}, L_{q}(\Omega)\right)} .
$$

(2) If $f \in L_{\infty}\left(\mathbb{R}, H_{q}^{1}(\Omega)\right)$ and $g \in L_{p}\left(\mathbb{R}, H_{q}^{1}(\Omega)\right)$, then

$$
\|f g\|_{L_{p}\left(\mathbb{R}, H_{q}^{1}(\Omega)\right)} \leq C\|f\|_{L_{\infty}\left(\mathbb{R}, H_{q}^{1}(\Omega)\right)}\|g\|_{L_{p}\left(\mathbb{R}, H_{q}^{1}(\Omega)\right)} .
$$


Proof. To prove the first assertion, we use the fact that

$$
H_{p}^{1 / 2}\left(\mathbb{R}, L_{q}(\Omega)\right)=\left(L_{p}\left(\mathbb{R}, L_{q}(\Omega)\right), H_{p}^{1}\left(\mathbb{R}, L_{q}(\Omega)\right)\right)_{[1 / 2]},
$$

where $(\cdot, \cdot)_{[\theta]}$ denotes a complex interpolation functor for $\theta \in(0,1)$. Since

$$
\begin{aligned}
\left\|\partial_{t}(f g)\right\|_{L_{p}\left(\mathbb{R}, L_{q}(\Omega)\right)} & \leq\|f\|_{H_{\infty}^{1}\left(\mathbb{R}, L_{\infty}(\Omega)\right)}\|g\|_{H_{p}^{1}\left(\mathbb{R}, L_{q}(\Omega)\right)}, \\
\|f g\|_{L_{p}\left(\mathbb{R}, L_{q}(\Omega)\right)} & \leq\|f\|_{L_{\infty}\left(\mathbb{R}, L_{\infty}(\Omega)\right)}\|g\|_{L_{p}\left(\mathbb{R}, L_{q}(\Omega)\right)}
\end{aligned}
$$

by (6.16) we have the first assertion. The second assertion follows immediately from the Banach algebra property of $H_{q}^{1}(\Omega)$ for $3<q<\infty$.

Recalling that $\mathbf{n}$ is defined on $\mathbb{R}^{3}$ and $\|\mathbf{n}\|_{H_{\infty}^{2}\left(\mathbb{R}^{3}\right)}<\infty$, by (6.9) we have

$$
\left\|\partial_{t} e_{T}\left[\mathcal{R}_{\mathbf{v}}\right]\right\|_{L_{\infty}\left(\mathbb{R}, L_{\infty}(\Omega)\right)} \leq C\|\mathbf{v}\|_{L_{\infty}\left((0, T), H_{\infty}^{1}(\Omega)\right)} \leq C\left(\mathcal{I}+<e^{\gamma t} V>_{T}\right) .
$$

By Sobolev's inequality and Hölder's inequality, we have

$$
\left\|e_{T}\left[\mathcal{R}_{\mathbf{v}}\right]\right\|_{L_{\infty}\left(\mathbb{R}, L_{\infty}(\Omega)\right)} \leq C \int_{0}^{T}\|\mathbf{v}(\cdot, s)\|_{H_{q}^{2}(\Omega)} d s \leq C\left(\int_{0}^{T}\left(e^{\gamma t}\|\mathbf{v}(\cdot, s)\|_{H_{q}^{2}(\Omega)}\right)^{p} d s\right)^{1 / p} \leq C<e^{\gamma t} V>_{T} .
$$

Noting that $\left|\mathbf{k}_{\mathbf{v}}\right| \leq \delta$, we also have

$$
\left\|e_{T}\left[\mathcal{R}_{\mathbf{v}}\right]\right\|_{L_{\infty}\left(\mathbb{R}, H_{q}^{1}(\Omega)\right)} \leq C<e^{\gamma t} V>_{T} .
$$

Thus, applying Lemma 11 and Lemma 7, we obtain

$$
\begin{aligned}
& \left\|E\left[e^{\gamma t} R_{4}(U)\right]\right\|_{L_{p}\left(\mathbb{R}, H_{q}^{1}(\Omega)\right)}+\left\|E\left[e^{\gamma t} R_{4}(U)\right]\right\|_{H_{p}^{1 / 2}\left(\mathbb{R}, L_{q}(\Omega)\right)} \\
& \quad \leq C\left(\mathcal{I}+<e^{\gamma t} V>_{T}\right)\left(\left\|\tilde{e}_{T}\left[e^{\gamma t} H\right]\right\|_{L_{p}\left(\mathbb{R}, H_{q}^{2}(\Omega)\right)}+\left\|\partial_{t} \tilde{e}_{T}\left[e^{\gamma t} H\right]\right\|_{L_{p}\left(\mathbb{R}, L_{q}(\Omega)\right)}\right) .
\end{aligned}
$$

Since $\left.e^{\gamma t} H\right|_{t=0}=\left.H\right|_{t=0}$, we have

$$
\tilde{e}_{T}\left[e^{\gamma t} H\right]=e_{T}\left[e^{\gamma t} H-T(t) \tilde{H}_{0}\right]+\psi(t) T(|t|) \tilde{H}_{0},
$$

where $\tilde{H}_{0}$ is a function in $B_{q, p}^{2(1-1 / p)}\left(\mathbb{R}^{N}\right)$ such that

$$
\tilde{H}_{0}=\left.H\right|_{t=0} \quad \text { in } \Omega, \quad\left\|\tilde{H}_{0}\right\|_{B_{q, p}^{2(1-1 / p)}\left(\mathbb{R}^{N}\right)} \leq C\left\|\left.H\right|_{t=0}\right\|_{B_{q, p}^{2(1-1 / p)}(\Omega)} .
$$

Thus, using (5.27) and (6.15), we get

$$
\begin{aligned}
& \left\|\tilde{e}_{T}\left[e^{\gamma t} H\right]\right\|_{L_{p}\left(\mathbb{R}, H_{q}^{2}(\Omega)\right)}+\left\|\partial_{t} \tilde{e}_{T}\left[e^{\gamma t} H\right]\right\|_{L_{p}\left(\mathbb{R}, L_{q}(\Omega)\right)} \\
& \quad \leq C\left(\left\|e^{\gamma t} \nabla h\right\|_{L_{p}\left((0, T), H_{q}^{1}(\Omega)\right)}+\left\|e^{\gamma t} \partial_{t} h\right\|_{L_{p}\left((0, T), L_{q}(\Omega)\right)}+\left\|\left.H\right|_{t=0}\right\|_{B_{q, p}^{2(1-1 / p)}(\Omega)}\right) .
\end{aligned}
$$

Finally, by Poincaré's inequality, we have

$$
\left\|\left.H\right|_{t=0}\right\|_{B_{q, p}^{2(1-1 / p)}(\Omega)}=\left\|\left.H\right|_{t=0}\right\|_{L_{q}(\Omega)}+\left\|\nabla\left(\left.H\right|_{t=0}\right)\right\|_{B_{q, p}^{1-2 / p}(\Omega)} \leq C\left\|\nabla h_{0}\right\|_{B_{q, p}^{1-2 / p}(\Omega)} .
$$

Summing up, we have obtained

$$
\left\|E\left[e^{\gamma t} R_{4}(U)\right]\right\|_{H_{p}^{1 / 2}\left(\mathbb{R}, L_{q}(\Omega)\right)}+\left\|E\left[e^{\gamma t} R_{4}(U)\right]\right\|_{L_{p}\left(\mathbb{R}, H_{q}^{1}(\Omega)\right)} \leq C\left(\mathcal{I}+<e^{\gamma t} V>_{T}\right)^{2} .
$$

Applying Theorem 10 to Eq. (6.3) and using the estimates (6.8), (6.12), (6.13), (6.14), and (6.17), we have

$$
<e^{\gamma t} V>_{T} \leq C\left(\mathcal{I}+\left(\mathcal{I}+<e^{\gamma t} V>_{T}\right)^{2}\right) .
$$

We assume that $\mathcal{I} \leq \epsilon<1$, and so $\left(\mathcal{I}+<e^{\gamma t} V>_{T}\right)^{2} \leq 2\left(\mathcal{I}+<e^{\gamma t} V>_{T}^{2}\right)$, which completes the proof of (6.2). 
We now prolong a local solution to $(0, \infty)$. Let $T>0$ and $\eta, \mathbf{v}$ and $\vartheta$ be solutions of Eq. (2.15) satisfying (6.1). Then, by (6.2) we have

$$
<e^{\gamma s} V>_{t} \leq C\left(\mathcal{I}+<e^{\gamma s} V>_{t}^{2}\right)
$$

for any $t \in(0, T)$, where $C$ is independent of $t \in(0, T)$ and $T>0$. Let $r_{ \pm}(\epsilon)$ be two roots of the quadratic equation: $C^{-1} x=\epsilon+x^{2}$, that is $r_{ \pm}(\epsilon)=(2 C)^{-1} \pm \sqrt{(2 C)^{-2}-\epsilon}$. We find a small positive number $\epsilon_{1}>0$ such that

$$
0<r_{-}(\epsilon) \leq 2 C \epsilon<2 C^{-1}<r_{+}(\epsilon)
$$

for $0<\epsilon<\epsilon_{1}$. Since $\left\langle e^{\gamma s} V>_{t}\right.$ satisfies the inequality (6.18), we have $\left\langle e^{\gamma s} V>_{t} \leq r_{-}(\epsilon)\right.$ or $\left\langle e^{\gamma s} V>_{t} \geq\right.$ $r_{+}(\epsilon)$. Since

$$
<e^{\gamma s} V>_{t} \rightarrow 0 \text { as } t \rightarrow 0,
$$

for small $t \in(0, T)$, we have $\left\langle e^{\gamma s} V>_{t} \leq r_{-}(\epsilon)\right.$. But, $\left\langle e^{\gamma s} V>_{t}\right.$ is continuous with respect to $t \in(0, T)$, and so $\left\langle e^{\gamma s} V>_{t} \leq r_{-}(\epsilon)\right.$ for any $t \in(0, T)$. Thus, we have

$$
<e^{\gamma s} V>_{T} \leq 2 C \epsilon
$$

By (6.5), (6.6), and (6.9), we see that there exists a constant $M>0$ for which

$$
\begin{gathered}
\left\|\eta-\rho_{0}\right\|_{L_{\infty}\left((0, T), H_{q}^{1}(\Omega)\right)} \leq M \epsilon, \quad\left\|\left(\mathbf{v}, \vartheta-h_{0}\right)\right\|_{L_{\infty}\left((0, T), B_{q, p}^{2(1-1 / p)}(\Omega)\right)} \leq M \epsilon, \\
\left\|\left(\rho_{1}, \rho_{2}\right)-\left(\rho_{1 *}, \rho_{2 *}\right)\right\|_{L_{\infty}\left((0, T), H_{q}^{1}(\Omega)\right)} \leq M \epsilon .
\end{gathered}
$$

Let $\eta^{\prime}, \mathbf{v}^{\prime}$ and $\vartheta^{\prime}$ be solutions of the following equations:

$$
\left\{\begin{aligned}
\partial_{t} \eta^{\prime}+\eta \operatorname{div} \mathbf{v}^{\prime}=R_{1}^{\prime}(U) & \text { in } \Omega \times\left(T, T+T_{1}\right), \\
\eta \partial_{t} \mathbf{v}^{\prime}-\mu \Delta \mathbf{v}^{\prime}-\nu \nabla \operatorname{div} \mathbf{v}^{\prime}+\frac{\eta}{\Sigma_{\rho^{\prime}}} \nabla \eta^{\prime}+\frac{\left(m_{1}-m_{2}\right) \rho_{1}^{\prime} \rho_{2}^{\prime}}{\Sigma_{\rho^{\prime}}} \nabla \vartheta^{\prime}=R_{2}^{\prime}(U) & \text { in } \Omega \times\left(T, T+T_{1}\right), \\
\frac{m_{1} m_{2} \rho_{1}^{\prime} \rho_{2}^{\prime}}{\Sigma_{\rho^{\prime}}} \partial_{t} \vartheta^{\prime}+\frac{\left(m_{1}-m_{2}\right) \rho_{1}^{\prime} \rho_{2}^{\prime}}{\Sigma_{\rho^{\prime}}} \operatorname{div} \mathbf{v}^{\prime}-\operatorname{div}\left(\frac{\rho_{1}^{\prime} \rho_{2}^{\prime}}{\mathfrak{p}^{\prime} \rho^{\prime}} \nabla \vartheta^{\prime}\right)=R_{3}^{\prime}(U) & \text { in } \Omega \times\left(T, T+T_{1}\right), \\
\mathbf{v}^{\prime}=0, \quad(\nabla \vartheta) \cdot \mathbf{n}=R_{4}^{\prime}(U) & \text { on } \Gamma \times\left(T, T+T_{1}\right), \\
\left.\left(\eta^{\prime}, \mathbf{v}^{\prime}, \vartheta^{\prime}\right)\right|_{t=T}=(\eta(\cdot, T), \mathbf{v}(\cdot, T), \vartheta(\cdot, T)) & \text { in } \Omega .
\end{aligned}\right.
$$

Here, $\Sigma_{\rho^{\prime}}=m_{1} \rho_{1}^{\prime}+m_{2} \rho_{2}^{\prime}, \mathfrak{p}^{\prime}=\rho_{1}^{\prime} / m_{1}+\rho_{2}^{\prime} / m_{2}$, and $R_{i}(U)$ are defined by replacing $\int_{0}^{t} \nabla \mathbf{v}(\cdot, s) d s, \eta, \rho_{1}$, $\rho_{2}, \rho \mathbf{v}$, and $\vartheta$ by $\int_{0}^{T} \nabla \mathbf{v}(\cdot, s) d s+\int_{T}^{t} \nabla \mathbf{v}^{\prime}(\cdot, s) d s, \eta^{\prime}, \rho_{1}^{\prime}, \rho_{2}^{\prime}, \rho^{\prime} \mathbf{v}^{\prime}$, and $\vartheta^{\prime}$. Employing the same argument as that in the proof of Theorem 10 we can show that there exists a $T_{1}$ depending on $\epsilon>0$ such that problem (6.21) admits unique solutions $\eta^{\prime}, \mathbf{v}^{\prime}$ and $\vartheta^{\prime}$ with

$$
\begin{gathered}
\eta^{\prime} \in H_{p}^{1}\left(\left(T, T+T_{1}\right), H_{q}^{1}(\Omega)\right), \quad \mathbf{v}^{\prime} \in H_{p}^{1}\left(\left(T, T+T_{1}\right), L_{q}(\Omega)^{3}\right) \cap L_{p}\left(\left(T, T+T_{1}\right), H_{q}^{2}(\Omega)^{3}\right), \\
\vartheta^{\prime} \in H_{p}^{1}\left(\left(T, T+T_{1}\right), L_{q}(\Omega)\right) \cap L_{p}\left(\left(T, T+T_{1}\right), H_{q}^{2}(\Omega)\right), \quad \int_{T}^{T+T_{1}}\left\|\nabla \mathbf{v}^{\prime}(\cdot, s)\right\|_{L_{\infty}(\Omega)} d s \leq \delta, \\
\rho_{*} / 4 \leq \eta^{\prime}(x, t) \leq 4 \rho_{*}, \quad\left|\vartheta^{\prime}(x, t)\right| \leq 4\left|h_{*}\right| \quad \text { for }(x, t) \in \Omega \times\left(T, T+T_{1}\right) .
\end{gathered}
$$

Because, choosing $\epsilon>0$ small enough, in view of (6.20) we may assume that

$$
\rho_{i *} / 2 \leq \rho_{i}(x, T) \leq 2 \rho_{i *} \quad \text { in } x \in \Omega \text { for } i=1,2 .
$$

Thus, setting

$$
f^{\prime \prime}= \begin{cases}f & \text { for } t \in(0, T), \\ f^{\prime} & \text { for } t \in\left(T, T+T_{1}\right),\end{cases}
$$

for $f \in\{\eta, \mathbf{v}, \vartheta\}, \eta^{\prime \prime}, \mathbf{v}^{\prime \prime}$, and $\vartheta^{\prime \prime}$ are solutions of Eq. (2.15) satisfying (6.1), where $T$ is replaced by $T+T_{1}$. The repeated use of this argument implies the existence of solutions $\eta, \mathbf{v}, \vartheta$ of Eq. (2.15) with $T=\infty$, which satisfies the estimate: $\left\langle e^{\gamma t} V>_{\infty} \leq C \epsilon\right.$. This completes the proof of Theorem 2 


\section{Maximal $L_{p}-L_{q}$ regularity - proof of Theorem 3}

In this section, we consider the linear problem (5.1) in a uniformly $C^{2}$ domain in the $N$-dimensional Euclidean space $\mathbb{R}^{N}(N \geq 2)$. To prove Theorem 3. we use the $\mathcal{R}$-bounded solution operators for the generalized resolvent problem corresponding to Eq. (5.1). We first make a definition.

Definition 12. Let $X$ and $Y$ be two Banach spaces, and $\|\cdot\|_{X}$ and $\|\cdot\|_{Y}$ their norms. A family of operators $\mathcal{T} \subset \mathcal{L}(X, Y)$ is called $\mathcal{R}$-bounded on $\mathcal{L}(X, Y)$ if there exist constants $C>0$ and $p \in[1, \infty)$ such that for any $n \in \mathbb{N},\left\{T_{j}\right\}_{j=1}^{n} \subset \mathcal{T}$ and $\left\{f_{j}\right\}_{j=1}^{n} \subset X$, the inequality

$$
\int_{0}^{1}\left\|\sum_{j=1}^{n} r_{j}(u) T_{j} f_{j}\right\|_{Y}^{p} d u \leq C \int_{0}^{1}\left\|\sum_{j=1}^{n} r_{j}(u) f_{j}\right\|_{X}^{p} d u .
$$

Here, the Rademacher functions $r_{j}:[0,1] \rightarrow\{-1,1\}, j \in \mathbb{N}$, are given by $r_{j}(t)=\operatorname{sign}\left(\sin \left(2^{j} \pi t\right)\right)$. The smallest such $C$ is called $\mathcal{R}$-bound of $\mathcal{T}$ on $\mathcal{L}(X, Y)$ which is written by $\mathcal{R}_{\mathcal{L}(X, Y)} \mathcal{T}$.

The generalized resolvent problem corresponding to Eq. (5.1) is the following system:

$$
\left\{\begin{aligned}
\lambda \zeta+\rho_{0}(x) \operatorname{div} \mathbf{v} & =f_{1} & & \text { in } \Omega \\
\rho_{0}(x) \lambda \mathbf{v}-\mu \Delta \mathbf{v}-\nu \nabla \operatorname{div} \mathbf{v}+\gamma_{1}(x) \nabla \zeta+\gamma_{2}(x) \nabla \vartheta & =\mathbf{f}_{2} & & \text { in } \Omega \\
\gamma_{3}(x) \lambda \vartheta+\gamma_{2}(x) \operatorname{div} \mathbf{v}-\operatorname{div}\left(\gamma_{4}(x) \nabla \vartheta\right) & =f_{3} & & \text { in } \Omega \\
\mathbf{v}=0, & (\nabla \vartheta) \cdot \mathbf{n}=f_{4} & & \text { on } \Gamma .
\end{aligned}\right.
$$

We assume that the coefficients $\rho_{0}(x), \gamma_{i}(x), \quad i=1, \ldots, 4$ are uniformly continous on $\bar{\Omega}$ and satisfy the conditions (5.2). The main part of this section is to prove the following theorem concerning the existence of $\mathcal{R}$-bounded solution operators for Eq. (7.1).

Theorem 13. Let $1<q<\infty$ and $0<\epsilon<\pi / 2$. Assume that $\Omega$ is a uniform $C^{2}$ domain. Let

$$
\begin{aligned}
& X_{q}(\Omega)=\left\{\left(f_{1}, \mathbf{f}_{2}, f_{3}, f_{4}\right) \mid f_{1}, f_{4} \in H_{q}^{1}(\Omega), \mathbf{f}_{2} \in L_{q}(\Omega)^{N}, \quad f_{3} \in L_{q}(\Omega)\right\}, \\
& \mathcal{X}_{q}(\Omega)=\left\{\left(F_{1}, F_{2}, F_{3}, F_{4}, F_{5}\right) \mid F_{1}, F_{5} \in H_{q}^{1}(\Omega), \quad F_{3}, F_{4} \in L_{q}(\Omega), \quad F_{2} \in L_{q}(\Omega)^{N}\right\} .
\end{aligned}
$$

Then, there exist a positive constant $\lambda_{0}$ and operator families $\mathcal{A}(\lambda) \in \operatorname{Hol}\left(\Sigma_{\epsilon, \lambda_{0}}, \mathcal{L}\left(\mathcal{X}_{q}(\Omega), H_{q}^{1}(\Omega)\right)\right)$, $\mathcal{B}_{1}(\lambda) \in \operatorname{Hol}\left(\Sigma_{\epsilon, \lambda_{0}}, \mathcal{L}\left(\mathcal{X}_{q}(\Omega), H_{q}^{2}(\Omega)^{N}\right)\right)$, and $\mathcal{B}_{2}(\lambda) \in \operatorname{Hol}\left(\Sigma_{\epsilon, \lambda_{0}}, \mathcal{L}\left(\mathcal{X}_{q}(\Omega), H_{q}^{2}(\Omega)\right)\right)$ such that for any $\left(f_{1}, \mathbf{f}_{2}, f_{3}, f_{4}\right) \in X_{q}(\Omega)$ and $\lambda \in \Sigma_{\epsilon, \lambda_{0}}, \zeta=\mathcal{A}(\lambda) F_{\lambda}, \mathbf{v}=\mathcal{B}_{1}(\lambda) F_{\lambda}$, and $\vartheta=\mathcal{B}_{2}(\lambda) F_{\lambda}$ are unique solutions of Eq. (7.1), where $F_{\lambda}=\left(f_{1}, \mathbf{f}_{2}, f_{3}, \lambda^{1 / 2} f_{4}, f_{4}\right)$, and

$$
\begin{array}{r}
\mathcal{R}_{\mathcal{L}\left(\mathcal{X}_{q}(\Omega), H_{q}^{1}(\Omega)\right)}\left(\left\{\left(\tau \partial_{\tau}\right)^{\ell} \mathcal{A}(\lambda) \mid \lambda \in \Sigma_{\epsilon, \lambda_{0}}\right\}\right) \leq r_{b}, \\
\mathcal{R}_{\mathcal{L}\left(\mathcal{X}_{q}(\Omega), H_{q}^{2-j}(\Omega)^{N}\right)}\left(\left\{\left(\tau \partial_{\tau}\right)^{\ell}\left(\lambda^{j / 2} \mathcal{B}_{1}(\lambda)\right) \mid \lambda \in \Sigma_{\epsilon, \lambda_{0}}\right\}\right) \leq r_{b}, \\
\mathcal{R}_{\mathcal{L}\left(\mathcal{X}_{q}(\Omega), H_{q}^{2-j}(\Omega)\right)}\left(\left\{\left(\tau \partial_{\tau}\right)^{\ell}\left(\lambda^{j / 2} \mathcal{B}_{2}(\lambda)\right) \mid \lambda \in \Sigma_{\epsilon, \lambda_{0}}\right\}\right) \leq r_{b}
\end{array}
$$

for $\ell=0,1, j=0,1,2$, and for some constant $r_{b}$.

Remark 14. $F_{1}, F_{2}, F_{3}, F_{4}$ and $F_{5}$ are variables corresponding to $f_{1}, f_{2}, f_{3}, \lambda^{1 / 2} f_{4}$ and $f_{4}$. The norm of $\mathcal{X}_{q}(\Omega)$ is defined by

$$
\left\|\left(F_{1}, F_{2}, F_{3}, F_{4}, F_{5}\right)\right\|_{\mathcal{X}_{q}(\Omega)}=\left\|\left(F_{1}, F_{5}\right)\right\|_{H_{q}^{1}(\Omega)}+\left\|\left(F_{2}, F_{3}, F_{4}\right)\right\|_{L_{q}(\Omega)} .
$$

Since we consider the case that $\lambda \in \Sigma_{\epsilon, \lambda_{0}}$ with $\lambda_{0}>0$, setting $\zeta=\lambda^{-1}\left(f_{1}-\rho_{0}(x)\right.$ div $\left.\mathbf{v}\right)$, and inserting this formula into the second equation in (7.1), we rewrite it as

$$
\rho_{0}(x) \lambda \mathbf{v}-\mu \Delta \mathbf{v}-\nu \nabla \operatorname{div} \mathbf{v}-\gamma_{1}(x) \lambda^{-1} \nabla\left(\rho_{0}(x) \operatorname{div} \mathbf{v}\right)+\gamma_{2}(x) \nabla \vartheta=\mathbf{f}_{2}-\lambda^{-1} \gamma_{1}(x) \nabla f_{1}
$$

Since $\gamma_{2}(x) \nabla \vartheta$ and $\gamma_{2}(x)$ div $\mathbf{v}$ are lower order terms, our main concern is to prove the existence of $\mathcal{R}$-bounded solution operators for the following two equations:

$$
\begin{gathered}
\rho_{0}(x) \lambda \mathbf{v}-\mu \Delta \mathbf{v}-\nu \nabla \operatorname{div} \mathbf{v}-\gamma_{1}(x) \lambda^{-1} \nabla\left(\rho_{0}(x) \operatorname{div} \mathbf{v}\right)=\mathbf{g} \quad \text { in } \Omega,\left.\quad \mathbf{v}\right|_{\Gamma}=0 ; \\
\gamma_{3}(x) \lambda \vartheta-\operatorname{div}\left(\gamma_{4}(x) \nabla \vartheta\right)=h_{1} \quad \text { in } \Omega,\left.\quad(\nabla \vartheta) \cdot \mathbf{n}\right|_{\Gamma}=h_{2} .
\end{gathered}
$$

Then, we shall prove the following theorem. 
Theorem 15. Let $1<q<\infty$ and $0<\epsilon<\pi / 2$. Assume that $\Omega$ is a uniform $C^{2}$ domain in $\mathbb{R}^{N}$. Then, there exists a positive constant $\lambda_{0}$ such that the following assertions hold:

(1) There exists an operator family $\mathcal{C}(\lambda) \in \operatorname{Hol}\left(\Sigma_{\epsilon, \lambda_{0}}, \mathcal{L}\left(L_{q}(\Omega)^{N}, H_{q}^{2}(\Omega)^{N}\right)\right)$ such that for any $\lambda \in \Sigma_{\epsilon, \lambda_{0}}$ and $\mathbf{g} \in L_{q}(\Omega)^{N}, \mathbf{v}=\mathcal{C}(\lambda) \mathbf{g}$ is a unique solution of $E q$. (17.3), and

$$
\mathcal{R}_{\mathcal{L}\left(L_{q}(\Omega)^{N}, H_{q}^{2-j}(\Omega)^{N}\right)}\left(\left\{\left(\tau \partial_{\tau}\right)^{\ell} \mathcal{C}(\lambda) \mid \lambda \in \Sigma_{\epsilon, \lambda_{0}}\right\}\right) \leq r_{b}
$$

for $\ell=0,1$ and $j=0,1,2$.

(2) Let $Y_{q}(\Omega)$ and $\mathcal{Y}_{q}(\Omega)$ be the spaces defined in Notation of Sect. 1 with $G=\Omega$. Then, there exists an operator family $\mathcal{D}(\lambda) \in \operatorname{Hol}\left(\Sigma_{\epsilon, \lambda_{0}}, \mathcal{L}\left(\mathcal{Y}_{q}(\Omega), H_{q}^{2}(\Omega)\right)\right.$ such that for any $\lambda \in \Sigma_{\epsilon, \lambda_{0}}$ and $\left(h_{1}, h_{2}\right) \in Y_{q}(\Omega), \vartheta=\mathcal{D}(\lambda)\left(h_{1}, \lambda^{1 / 2} h_{2}, h_{2}\right)$ is a unique solution of Eq. (17.4), and

$$
\mathcal{R}_{\mathcal{L}\left(\mathcal{Y}_{q}(\Omega), H_{q}^{2-j}(\Omega)\right)}\left(\left\{\left(\tau \partial_{\tau}\right)^{\ell} \mathcal{D}(\lambda) \mid \lambda \in \Sigma_{\epsilon, \lambda_{0}}\right\}\right) \leq r_{b}
$$

for $\ell=0,1$ and $j=0,1,2$.

\subsection{The model problems in $\mathbb{R}^{N}$ and $\mathbb{R}_{+}^{N}$}

First, we consider the model problem in $\mathbb{R}^{N}$. In what follows, let $\rho_{0 *}, \gamma_{1 *}, \gamma_{3 *}$ and $\gamma_{4 *}$ be positive constants. Assume that there exist two positive constants $b_{1}$ and $b_{2}$ for which

$$
b_{1} \leq \rho_{0 *}, \gamma_{* 1}, \gamma_{3 *}, \gamma_{4 *} \leq b_{2}
$$

Let us consider the following problems:

$$
\begin{gathered}
\rho_{0 *} \lambda \mathbf{v}-\mu \Delta \mathbf{v}-\nu \nabla \operatorname{div} \mathbf{v}-\gamma_{1 *} \rho_{0 *} \lambda^{-1} \nabla \operatorname{div} \mathbf{v}=\mathbf{g} \text { in } \mathbb{R}^{N} \\
\gamma_{3 *} \lambda \vartheta-\gamma_{4 *} \Delta \vartheta=h \quad \text { in } \mathbb{R}^{N} .
\end{gathered}
$$

Theorem 16. Let $1<q<\infty$ and $0<\epsilon<\pi / 2$. Then, we have the following assertions:

(1) There exist a large constant $\lambda_{0}>0$ and an operator family $\mathcal{C}_{1}(\lambda)$ with

$$
\mathcal{C}_{1}(\lambda) \in \operatorname{Hol}\left(\Sigma_{\epsilon, \lambda_{0}}, \mathcal{L}\left(L_{q}\left(\mathbb{R}^{N}\right)^{N}, H_{q}^{2}\left(\mathbb{R}^{N}\right)^{N}\right)\right)
$$

such that for any $\mathbf{g} \in L_{q}\left(\mathbb{R}^{N}\right)^{N}$ and $\lambda \in \Sigma_{\epsilon, \lambda_{0}}, \mathbf{v}=\mathcal{C}_{1}(\lambda) \mathbf{g}$ is a unique solution of Eq. (17.6), and

$$
\mathcal{R}_{\mathcal{L}\left(L_{q}\left(\mathbb{R}^{N}\right)^{N}, H_{q}^{2-j}\left(\mathbb{R}^{N}\right)^{N}\right)}\left(\left\{\left(\tau \partial_{\tau}\right)^{\ell} \mathcal{C}_{1}(\lambda) \mid \lambda \in \Sigma_{\epsilon, \lambda_{0}}\right\}\right) \leq r_{b 1}
$$

for $\ell=0,1$ and $j=0,1,2$. Here, $\lambda_{0}$ and $r_{1 b}$ depend solely on $N, q, \mu, \nu, b_{1}$ and $b_{2}$.

(2) Let $\lambda_{0} \geq 1$. Then, there exists an operator family $\mathcal{D}_{1}(\lambda) \in \operatorname{Hol}\left(\Sigma_{\epsilon, \lambda_{0}}, \mathcal{L}\left(L_{q}\left(\mathbb{R}^{N}\right), H_{q}^{2}\left(\mathbb{R}^{N}\right)\right)\right.$ such that for any $h \in Y_{q}\left(\mathbb{R}^{N}\right)$ and $\lambda \in \Sigma_{\epsilon, \lambda_{0}}, \vartheta=\mathcal{D}_{1}(\lambda) h$ is a unique solution of Eq. (7.7), and

$$
\mathcal{R}_{\mathcal{L}\left(L_{q}\left(\mathbb{R}^{N}\right), H_{q}^{2-j}\left(\mathbb{R}^{N}\right)\right)}\left(\left\{\left(\tau \partial_{\tau}\right)^{\ell} \mathcal{D}_{1}(\lambda) \mid \lambda \in \Sigma_{\epsilon, \lambda_{0}}\right\}\right) \leq r_{b 2}
$$

for $\ell=0,1$ and $j=0,1,2$. Here, $r_{b 2}$ depends solely on $N, q, \lambda_{0}, b_{1}$ and $b_{2}$.

Proof. The assertion (1) was proved in Enomoto-Shibata [12, Theorem 3.2], and so we may omit the proof. To prove (2), using the Fourier tranform $\mathcal{F}$ and its inversion formula $\mathcal{F}^{-1}$, we define $\vartheta$ by

$$
\vartheta=\mathcal{F}^{-1}\left[\frac{\mathcal{F}[h](\xi)}{\gamma_{3 *} \lambda+\gamma_{4 *}|\xi|^{2}}\right](x) .
$$

Thus, by Lemma 3.1 and Theorem 3.3 in [12], we can show the assertion (2). Thus, we also may omit the detailed proof. 
Next we consider the half space problem. Let

$$
\mathbb{R}_{+}^{N}=\left\{x=\left(x_{1}, \ldots, x_{N}\right) \in \mathbb{R}^{N} \mid x_{N}>0\right\}, \quad \mathbb{R}_{0}^{N}=\left\{x=\left(x_{1}, \ldots, x_{N}\right) \in \mathbb{R}^{N} \mid x_{N}=0\right\},
$$

and $\mathbf{n}_{0}={ }^{\top}(0, \ldots, 0,-1)$. We consider the following problems in $\mathbb{R}_{+}^{N}$ :

$$
\begin{gathered}
\rho_{0 *} \lambda \mathbf{v}-\mu \Delta \mathbf{v}-\nu \nabla \operatorname{div} \mathbf{v}-\gamma_{1 *} \rho_{0 *} \lambda^{-1} \nabla \operatorname{div} \mathbf{v}=\mathbf{g} \quad \text { in } \mathbb{R}_{+}^{N},\left.\quad \mathbf{v}\right|_{\mathbb{R}_{0}^{N}}=0 \\
\gamma_{3 *} \lambda \vartheta-\gamma_{4 *} \Delta \vartheta=h_{1} \quad \text { in } \mathbb{R}_{+}^{N}, \quad(\nabla \vartheta) \cdot \mathbf{n}_{0}=h_{2} \quad \text { on } \mathbb{R}_{0}^{N} .
\end{gathered}
$$

Theorem 17. Let $1<q<\infty, 0<\epsilon<\pi / 2$, and $\lambda_{0} \geq 1$.

(1) There exist a large constant $\lambda_{0}>0$ and an operator family $\mathcal{C}_{2}(\lambda)$ with

$$
\mathcal{C}_{2}(\lambda) \in \operatorname{Hol}\left(\Sigma_{\epsilon, \lambda_{0}}, \mathcal{L}\left(L_{q}\left(\mathbb{R}_{+}^{N}\right)^{N}, H_{q}^{2}\left(\mathbb{R}_{+}^{N}\right)^{N}\right)\right.
$$

such that for any $\mathbf{g} \in L_{q}\left(\mathbb{R}_{+}^{N}\right)^{N}$ and $\lambda \in \Sigma_{\epsilon, \lambda_{0}}, \mathbf{v}=\mathcal{C}_{2}(\lambda) \mathbf{g}$ is a unique solution of $E q$. (7.8), and

$$
\mathcal{R}_{\mathcal{L}\left(L_{q}\left(\mathbb{R}_{+}^{N}\right)^{N}, H_{q}^{2-j}\left(\mathbb{R}_{+}^{N}\right)^{N}\right)}\left(\left\{\left(\tau \partial_{\tau}\right)^{\ell} \mathcal{C}_{2}(\lambda) \mid \lambda \in \Sigma_{\epsilon, \lambda_{0}}\right\}\right) \leq r_{b}
$$

for $\ell=0,1$ and $j=0,1,2$.

(2) Let $\lambda_{0} \geq 1$. Then, there exists an operator family $\mathcal{D}_{2}(\lambda) \in \operatorname{Hol}\left(\Sigma_{\epsilon, \lambda_{0}}, \mathcal{L}\left(\mathcal{Y}_{q}\left(\mathbb{R}_{+}^{N}\right), H_{q}^{2}\left(\mathbb{R}_{+}^{N}\right)\right)\right.$ such that for any $\left(h_{1}, h_{2}\right) \in Y_{q}\left(\mathbb{R}_{+}^{N}\right)$ and $\lambda \in \Sigma_{\epsilon, \lambda_{0}}, \vartheta=\mathcal{D}_{2}(\lambda)\left(h_{1}, \lambda^{1 / 2} h_{2}, h_{2}\right)$ is a unique solution of Eq. (7.9), and

$$
\mathcal{R}_{\mathcal{L}\left(\mathcal{Y}_{q}\left(\mathbb{R}_{+}^{N}\right), H_{q}^{2-j}\left(\mathbb{R}_{+}^{N}\right)\right)}\left(\left\{\left(\tau \partial_{\tau}\right)^{\ell} \mathcal{D}_{2}(\lambda) \mid \lambda \in \Sigma_{\epsilon, \lambda_{0}}\right\}\right) \leq r_{b}
$$

for $\ell=0,1$ and $j=0,1,2$.

Here, $Y_{q}\left(\mathbb{R}_{+}^{N}\right)$ and $\mathcal{Y}_{q}\left(\mathbb{R}_{+}^{N}\right)$ are spaces defined in Sect. 1 with $G=\mathbb{R}_{+}^{N}$, and $r_{b}$ is a constant depending solely on $N, q, \lambda_{0}, b_{1}$ and $b_{2}$.

Proof. The first assertion has been proved in [12, Theorem 4.1]. To prove the second one we divide a solution of (7.9) into two parts: $\vartheta=\vartheta_{1}+\vartheta_{2}$, where $\vartheta_{1}$ and $\vartheta_{2}$ are solutions of the following problems:

$$
\begin{array}{llll}
\gamma_{3 *} \lambda \vartheta_{1}-\gamma_{4 *} \Delta \vartheta_{1}=h_{1} & \text { in } \mathbb{R}_{+}^{N}, & \left(\nabla \vartheta_{1}\right) \cdot \mathbf{n}_{0}=0 & \text { on } \mathbb{R}_{0}^{N} ; \\
\gamma_{3 *} \lambda \vartheta_{2}-\gamma_{4 *} \Delta \vartheta_{2}=0 & \text { in } \mathbb{R}_{+}^{N}, & \left(\nabla \vartheta_{2}\right) \cdot \mathbf{n}_{0}=h_{2} & \text { on } \mathbb{R}_{0}^{N} .
\end{array}
$$

Given function $F_{1}$ defined on $\mathbb{R}_{+}^{N}$, let $F_{1}^{e}$ be the even extension of $F_{1}$ to $x_{N}<0$, that is $F_{1}^{e}(x)=F_{1}(x)$ for $x_{N}>0$ and $F_{1}^{e}(x)=F_{1}\left(x^{\prime},-x_{N}\right)$ for $x_{N}<0$, where $x^{\prime}=\left(x_{1}, \ldots, x_{N-1}\right)$. We then define an $\mathcal{R}$ bounded solution operator $\mathcal{D}_{21}(\lambda)$ acting on $F_{1} \in L_{q}\left(\mathbb{R}_{+}^{N}\right)$ by

$$
\mathcal{D}_{21}(\lambda)\left[F_{1}\right]=\mathcal{F}^{-1}\left[\frac{\mathcal{F}\left[F_{1}^{e}\right](\xi)}{\gamma_{3 *} \lambda+\gamma_{4 *}|\xi|^{2}}\right] .
$$

Obviously, $\vartheta_{1}=\mathcal{D}_{1}(\lambda)\left[h_{1}\right]$ is a unique solution of Eq. (7.10).

To construct an $\mathcal{R}$ bounded solution operator for Eq. (7.11), we introduce the partial Fourier transform $\mathcal{F}^{\prime}$ and its inversion formula $\mathcal{F}_{\xi^{\prime}}^{-1}$, which are defined by

$$
\begin{aligned}
& \hat{f}\left(\xi^{\prime}, x_{N}\right)=\mathcal{F}^{\prime}[f]\left(\xi^{\prime}, x_{N}\right)=\int_{\mathbb{R}^{N-1}} e^{-i x^{\prime} \cdot \xi^{\prime}} f\left(x^{\prime}, x_{N}\right) d x^{\prime}, \\
& \mathcal{F}_{\xi^{\prime}}^{-1}\left[g\left(\xi^{\prime}, x_{N}\right)\right]\left(x^{\prime}\right)=\frac{1}{(2 \pi)^{N-1}} \int_{\mathbb{R}^{N-1}} e^{i x^{\prime} \cdot \xi^{\prime}} g\left(\xi^{\prime}, x_{N}\right) d \xi^{\prime},
\end{aligned}
$$

where $\xi^{\prime}=\left(\xi_{1}, \ldots, \xi_{N-1}\right) \in \mathbb{R}^{N-1}$ and $x^{\prime} \cdot \xi^{\prime}=\sum_{j=1}^{N-1} x_{j} \xi_{j}$. Applying the partial Fourier transform to (7.11), we have

$$
\left(\gamma_{3 *} \lambda+\gamma_{4 *}\left|\xi^{\prime}\right|^{2}\right) \hat{\vartheta}-\gamma_{* 4} \partial_{N}^{2} \hat{\vartheta}=0 \quad \text { for } x_{N}>0,\left.\quad \partial_{N} \vartheta\right|_{x_{N}=0}=-\hat{h}_{2}\left(\xi^{\prime}, 0\right),
$$


where $\left|\xi^{\prime}\right|^{2}=\sum_{j=1}^{N-1} \xi_{j}^{2}$ and $\partial_{N}=\partial / \partial_{N}$. Thus, $\vartheta_{2}$ is given by

$$
\begin{aligned}
\vartheta_{2} & =\mathcal{F}^{-1}\left[\frac{e^{-\sqrt{\gamma_{3 *} \gamma_{4 *}^{-1} \lambda+\left|\xi^{\prime}\right|^{2}} x_{N}}}{\sqrt{\gamma_{3 *} \gamma_{4 *}^{-1} \lambda+\left|\xi^{\prime}\right|^{2}}} \hat{h}_{2}\left(\xi^{\prime}, 0\right)\right]\left(x^{\prime}\right) \\
& =\int_{0}^{\infty} \mathcal{F}_{\xi^{\prime}}^{-1}\left[\frac{e^{-\sqrt{\gamma_{3 *} \gamma_{4 *}^{-1} \lambda+\left|\xi^{\prime}\right|^{2}}}\left(x_{N}+y_{N}\right)}{\sqrt{\gamma_{3 *} \gamma_{4 *}^{-1} \lambda+\left|\xi^{\prime}\right|^{2}}} \sqrt{\gamma_{3 *} \gamma_{4 *}^{-1} \lambda+\left|\xi^{\prime}\right|^{2}} \hat{h}_{2}\left(\xi^{\prime}, y_{N}\right)\right]\left(x^{\prime}\right) \\
& -\int_{0}^{\infty} \mathcal{F}_{\xi^{\prime}}^{-1}\left[\frac{e^{-\sqrt{\gamma_{3 *} \gamma_{4 *}^{-1} \lambda+\left|\xi^{\prime}\right|^{2}}\left(x_{N}+y_{N}\right)}}{\sqrt{\gamma_{3 *} \gamma_{4 *}^{-1} \lambda+\left|\xi^{\prime}\right|^{2}}} \partial_{N} \hat{h}_{2}\left(\xi^{\prime}, y_{N}\right)\right]\left(x^{\prime}\right) .
\end{aligned}
$$

Writing

$$
\sqrt{\gamma_{3 *} \gamma_{4 *}^{-1} \lambda+\left|\xi^{\prime}\right|^{2}}=\frac{\gamma_{3 *} \gamma_{4 *}^{-1} \lambda}{\sqrt{\gamma_{3 *} \gamma_{4 *}^{-1} \lambda+\left|\xi^{\prime}\right|^{2}}}-\sum_{j=1}^{N-1} \frac{i \xi_{j} i \xi_{j}}{\sqrt{\gamma_{3 *} \gamma_{4 *}^{-1} \lambda+\left|\xi^{\prime}\right|^{2}}}
$$

we have

$$
\begin{aligned}
& \int_{0}^{\infty} \mathcal{F}_{\xi^{\prime}}^{-1}\left[\frac{e^{-\sqrt{\gamma_{3 *} \gamma_{4 *}^{-1} \lambda+\left|\xi^{\prime}\right|^{2}}\left(x_{N}+y_{N}\right)}}{\sqrt{\gamma_{3 *} \gamma_{4 *}^{-1} \lambda+\left|\xi^{\prime}\right|^{2}}} \sqrt{\gamma_{3 *} \gamma_{4 *}^{-1} \lambda+\left|\xi^{\prime}\right|^{2}} \hat{h}_{2}\left(\xi^{\prime}, y_{N}\right)\right]\left(x^{\prime}\right) \\
& =\int_{0}^{\infty} \mathcal{F}_{\xi^{\prime}}^{-1}\left[\lambda^{1 / 2} e^{-\sqrt{\gamma_{3 *} \gamma_{4 *}^{-1} \lambda+\left|\xi^{\prime}\right|^{2}}\left(x_{N}+y_{N}\right)} \frac{\gamma_{3 *} \gamma_{4 *}^{-1} \lambda^{1 / 2}}{\sqrt{\gamma_{3 *} \gamma_{4 *}^{-1} \lambda+\left|\xi^{\prime}\right|^{2}}} \hat{h}_{2}\left(\xi^{\prime}, y_{N}\right)\right]\left(x^{\prime}\right) \\
& -\sum_{j=1}^{N-1} \int_{0}^{\infty} \mathcal{F}_{\xi^{\prime}}^{-1}\left[\left|\xi^{\prime}\right| e^{-\sqrt{\gamma_{3 *} \gamma_{4 *}^{-1} \lambda+\left|\xi^{\prime}\right|^{2}}\left(x_{N}+y_{N}\right)} \frac{i \xi_{j}}{\left|\xi^{\prime}\right| \sqrt{\gamma_{3 *} \gamma_{4 *}^{-1} \lambda+\left|\xi^{\prime}\right|^{2}}} \mathcal{F}^{\prime}\left[\partial_{j} h_{2}\right]\left(\xi^{\prime}, y_{N}\right)\right]\left(x^{\prime}\right) .
\end{aligned}
$$

We then define an operator $\mathcal{D}_{22}(\lambda)$ acting on $\left(F_{2}, F_{3}\right) \in L_{q}\left(\mathbb{R}_{+}^{N} \times H_{q}^{1}\left(\mathbb{R}_{+}^{N}\right)\right.$ by

$$
\begin{aligned}
\mathcal{D}_{22}(\lambda)\left(F_{2}, F_{3}\right) & =\int_{0}^{\infty} \mathcal{F}_{\xi^{\prime}}^{-1}\left[\lambda^{1 / 2} e^{-\sqrt{\gamma_{3 *} \gamma_{4 *}^{-1} \lambda+\left|\xi^{\prime}\right|^{2}}\left(x_{N}+y_{N}\right)} \frac{\gamma_{3 *} \gamma_{4 *}^{-1}}{\sqrt{\gamma_{3 *} \gamma_{4 *}^{-1} \lambda+\left|\xi^{\prime}\right|^{2}}} \mathcal{F}^{\prime}\left[F_{2}\right]\left(\xi^{\prime}, y_{N}\right)\right]\left(x^{\prime}\right) \\
& -\sum_{j=1}^{N-1} \int_{0}^{\infty} \mathcal{F}_{\xi^{\prime}}^{-1}\left[\left|\xi^{\prime}\right| e^{-\sqrt{\gamma_{3 *} \gamma_{4 *}^{-1} \lambda+\left|\xi^{\prime}\right|^{2}}\left(x_{N}+y_{N}\right)} \frac{i \xi_{j}}{\left|\xi^{\prime}\right| \sqrt{\gamma_{3 *} \gamma_{4 *}^{-1} \lambda+\left|\xi^{\prime}\right|^{2}}} \mathcal{F}^{\prime}\left[\partial_{j} F_{3}\right]\left(\xi^{\prime}, y_{N}\right)\right]\left(x^{\prime}\right) \\
& -\int_{0}^{\infty} \mathcal{F}_{\xi^{\prime}}^{-1}\left[\frac{e^{-\sqrt{\gamma_{3 *} \gamma_{4 *}^{-1} \lambda+\left|\xi^{\prime}\right|^{2}}\left(x_{N}+y_{N}\right)}}{\sqrt{\gamma_{3 *} \gamma_{4 *}^{-1} \lambda+\left|\xi^{\prime}\right|^{2}}} \mathcal{F}^{\prime}\left[\partial_{N} F_{3}\right]\left(\xi^{\prime}, y_{N}\right)\right]\left(x^{\prime}\right) .
\end{aligned}
$$

Obviously, $\vartheta_{2}=\mathcal{D}_{2}(\lambda)\left(\lambda^{1 / 2} h_{2}, h_{2}\right)$. Moreover, the $\mathcal{R}$ boundedness of the operator $\mathcal{D}_{22}(\lambda)$ follows from Lemma 4.2 in [12. This completes the proof of the assertion (2).

\subsection{Problem in a bent half space}

Let $\Phi: \mathbb{R}^{N} \rightarrow \mathbb{R}^{N}$ be a bijection of $C^{2}$ class and let $\Phi^{-1}$ be its inverse map. Writing $\nabla \Phi=\mathcal{A}+B(x)$ and $\nabla \Phi^{-1}=\mathcal{A}_{-}+B_{-}(x)$, we assume that $\mathcal{A}$ and $\mathcal{A}_{-}$are orthogonal matrices with constant coefficients and $B(x)$ and $B_{-}(x)$ are matrices of functions in $C^{1}\left(\mathbb{R}^{N}\right)$ with $N<r<\infty$ such that

$$
\left\|\left(B, B_{-}\right)\right\|_{L_{\infty}\left(\mathbb{R}^{N}\right)} \leq M_{1}, \quad\left\|\nabla\left(B, B_{-}\right)\right\|_{L_{\infty}\left(\mathbb{R}^{N}\right)} \leq M_{2} .
$$

We will choose $M_{1}$ small enough eventually, and so we may assume that $0<M_{1} \leq 1 \leq M_{2}$ in the following. Set $\Omega_{+}=\Phi\left(\mathbb{R}_{+}^{N}\right)$ and $\Gamma_{+}=\Phi\left(\mathbb{R}_{0}^{N}\right)$. Let $\mathbf{n}_{+}$be the unit outer normal to $\Gamma_{+}$. Since $\Gamma_{+}$is represented by $\Phi_{-1, N}(y)=0$, where $\Phi^{-1}={ }^{\top}\left(\Phi_{-1,1}, \ldots, \Phi_{-1, N}\right), \mathbf{n}_{+}$is given by

$$
\mathbf{n}_{+}=-\frac{\nabla \Phi_{-1, N}}{\left|\nabla \Phi_{-1, N}\right|}=-\frac{\top\left(\mathcal{A}_{N 1}+B_{N 1}, \ldots, \mathcal{A}_{N N}+B_{N N}\right)}{\sqrt{\sum_{j=1}^{N}\left(A_{N j}+B_{N j}\right)^{2}}} .
$$


Choosing $M_{1}>0$ small enough, by (7.12) we have

$$
\mathbf{n}_{+}=-{ }^{\top}\left(\mathcal{A}_{N 1}, \ldots, \mathcal{A}_{N N}\right)+\tilde{\mathbf{n}}_{+}
$$

where $\tilde{\mathbf{n}}_{+}$has the estimates:

$$
\left\|\tilde{\mathbf{n}}_{+}\right\|_{L_{\infty}\left(\mathbb{R}^{N}\right)} \leq C_{N} M_{1}, \quad\left\|\nabla \tilde{\mathbf{n}}_{+}\right\|_{L_{\infty}\left(\mathbb{R}^{N}\right)} \leq C_{M_{2}} .
$$

We consider the following two equations:

$$
\begin{gathered}
\rho_{0 *} \lambda \mathbf{v}-\mu \Delta \mathbf{v}-\nu \nabla \operatorname{div} \mathbf{v}-\gamma_{1 *} \rho_{0 *} \lambda^{-1} \nabla \operatorname{div} \mathbf{v}=\mathbf{g} \quad \text { in } \Omega_{+},\left.\quad \mathbf{v}\right|_{\Gamma_{+}}=0 ; \\
\gamma_{3 *} \lambda \vartheta-\gamma_{4 *} \Delta \vartheta=h_{1} \quad \text { in } \Omega_{+}, \quad(\nabla \vartheta) \cdot \mathbf{n}_{0}=h_{2} \quad \text { on } \Gamma_{+} .
\end{gathered}
$$

Theorem 18. Let $1<q<\infty$ and $0<\epsilon<\pi / 2$. Then, we have the following assertions:

(1) There exist a large constant $\lambda_{0}>0$ and an operator family $\mathcal{C}_{3}(\lambda)$ with

$$
\mathcal{C}_{3}(\lambda) \in \operatorname{Hol}\left(\Sigma_{\epsilon, \lambda_{0}}, \mathcal{L}\left(L_{q}\left(\Omega_{+}\right)^{N}, H_{q}^{2}\left(\Omega_{+}\right)^{N}\right)\right.
$$

such that for any $\mathbf{g} \in L_{q}\left(\Omega_{+}\right)^{N}$ and $\lambda \in \Sigma_{\epsilon, \lambda_{0}}, \mathbf{v}=\mathcal{C}_{3}(\lambda) \mathbf{g}$ is a unique solution of $E q$. (7.16), and

$$
\mathcal{R}_{\mathcal{L}\left(L_{q}\left(\Omega_{+}\right)^{N}, H_{q}^{2-j}\left(\Omega_{+}\right)^{N}\right)}\left(\left\{\left(\tau \partial_{\tau}\right)^{\ell} \mathcal{C}_{3}(\lambda) \mid \lambda \in \Sigma_{\epsilon, \lambda_{0}}\right\}\right) \leq r_{b 1}
$$

for $\ell=0,1$ and $j=0,1,2$. Here, $r_{b 1}$ is a constant depending solely on $N, q, \mu, \nu, b_{1}$ and $b_{2}$.

(2) Let $Y_{q}\left(\Omega_{+}\right)$and $\mathcal{Y}_{q}\left(\Omega_{+}\right)$be spaces defined by replacing $\Omega$ by $\Omega_{+}$in Theorem 15 . Then, there exist a positive constant $\lambda_{0}$ and an operator family $\mathcal{D}_{3}(\lambda) \in \operatorname{Hol}\left(\Sigma_{\epsilon, \lambda_{0}}, \mathcal{L}\left(\mathcal{Y}_{q}\left(\Omega_{+}\right), H_{q}^{2}\left(\Omega_{+}\right)\right)\right.$such that for any $\left(h_{1}, h_{2}\right) \in Y_{q}\left(\Omega_{+}\right)$and $\lambda \in \Sigma_{\epsilon, \lambda_{0}}, \vartheta=\mathcal{D}_{3}(\lambda)\left(h_{1}, \lambda^{1 / 2} h_{2}, h_{2}\right)$ is a unique solution of Eq. (7.17), and

$$
\mathcal{R}_{\mathcal{L}\left(L_{q}\left(\Omega_{+}\right), H_{q}^{2-j}\left(\Omega_{+}\right)\right)}\left(\left\{\left(\tau \partial_{\tau}\right)^{\ell} \mathcal{D}_{2}(\lambda) \mid \lambda \in \Sigma_{\epsilon, \lambda_{0}}\right\}\right) \leq r_{b 2}
$$

for $\ell=0,1$ and $j=0,1,2$. Here, $r_{b 2}$ is a constant depending solely on $N, q, b_{1}$ and $b_{2}$.

Proof. The first assertion was proved in Enomoto-Shibata [12, Theorem 5.1], and so we may omit the proof. Thus, we prove the assertion (2) below. For this purpose, we shall transform (7.17) into the equations in $\mathbb{R}_{+}^{N}$ by the change of variables: $x=\Phi^{-1}(y)$ with $x \in \mathbb{R}_{+}^{N}$ and $y \in \Omega_{+}$. We have

$$
\frac{\partial}{\partial y_{j}}=\sum_{k=1}^{N}\left(\mathcal{A}_{k j}+B_{k j}(x)\right) \frac{\partial}{\partial x_{k}}
$$

where $\mathcal{A}_{k j}$ is the $(k, j)^{\text {th }}$ component of $\mathcal{A}_{-}$and $B_{k j}(x)$ is the $(k, j)^{\text {th }}$ component of $B_{-}(\Phi(x))$. Let $\varphi(x)=\vartheta(\Phi(x))$ in (7.17), and then by (7.14) and (7.18) we have

$$
\gamma_{3 *} \lambda \varphi-\gamma_{4 *}\left[\Delta \varphi+A_{1} \nabla^{2} \varphi+A_{2} \nabla \varphi\right]=H_{1} \quad \text { in } \mathbb{R}_{+}^{N}, \quad(\nabla \varphi) \cdot \mathbf{n}_{0}+(\nabla \varphi) \cdot \mathbf{n}_{1}=H_{2} \quad \text { on } \mathbb{R}_{0}^{N} .
$$

Here, we have set

$$
\begin{aligned}
A_{1} \nabla^{2} \varphi & =\sum_{j, k, \ell=1}^{N}\left(\mathcal{A}_{k j} B_{\ell j}(x)+\mathcal{A}_{\ell j} B_{k j}(x)+B_{k j}(x) B_{\ell j}(x)\right) \frac{\partial^{2} \varphi}{\partial x_{k} \partial_{\ell}} \\
A_{2} \nabla \varphi & =\sum_{j, k, \ell=1}^{N}\left(\mathcal{A}_{k j}+B_{k j}(x)\right)\left(\frac{\partial}{\partial x_{k}} B_{\ell j}(x)\right) \frac{\partial \varphi}{\partial x_{\ell}} \\
(\nabla \varphi) \cdot \mathbf{n}_{1} & =\sum_{j, k=1}^{N}\left(\mathcal{A}_{N j} B_{k j}(x)+\tilde{n}_{j}(x) \mathcal{A}_{k j}+\tilde{n}_{j}(x) B_{k j}(x)\right) \frac{\partial \varphi}{\partial x_{k}} .
\end{aligned}
$$


Notice that

$$
\begin{aligned}
&\left\|A_{1} \nabla^{2} \varphi\right\|_{L_{q}\left(\mathbb{R}_{+}^{N}\right)} \leq C M_{1}\left\|\nabla^{2} \varphi\right\|_{L_{q}\left(\mathbb{R}_{+}^{N}\right)}, \\
&\left\|A_{2} \nabla \varphi\right\|_{L_{q}\left(\mathbb{R}_{+}^{N}\right)} \leq C_{M_{2}}\|\nabla \varphi\|_{L_{q}\left(\mathbb{R}_{+}^{N}\right)}, \\
&\left\|(\nabla \varphi) \cdot \mathbf{n}_{1}\right\|_{L_{q}\left(\mathbb{R}_{+}^{N}\right)} \leq C M_{1}\|\nabla \varphi\|_{L_{q}\left(\mathbb{R}_{+}^{N}\right)}, \\
&\left\|(\nabla \varphi) \cdot \mathbf{n}_{1}\right\|_{H_{q}^{1}\left(\mathbb{R}_{+}^{N}\right)} \leq C\left(M_{1}\left\|\nabla^{2} \varphi\right\|_{L_{q}\left(\mathbb{R}_{+}^{N}\right)}+C_{M_{2}}\|\nabla \varphi\|_{L_{q}\left(\mathbb{R}_{+}^{N}\right)}\right) .
\end{aligned}
$$

Let $\mathcal{C}_{2}(\lambda)$ be an $\mathcal{R}$-bounded solution operator given in Theorem 17 and set $\psi=\mathcal{C}_{2}(\lambda) F_{\lambda}\left(H_{1}, H_{2}\right)$. Here and in the following, $F_{\lambda}$ is an operator acting on $\left(H_{1}, H_{2}\right) \in Y_{q}\left(\mathbb{R}_{+}^{N}\right)$ defined by $F_{\lambda}\left(H_{1}, H_{2}\right)=$ $\left(H_{1}, \lambda^{1 / 2} H_{2}, H_{2}\right) \in \mathcal{Y}_{q}\left(\mathbb{R}_{+}^{N}\right)$. We then have

$$
\begin{aligned}
\gamma_{3 *} \lambda \psi-\gamma_{4 *}\left(\Delta \psi+A_{1} \nabla^{2} \psi+A_{2} \nabla \psi\right) & =H_{1}+R_{1}(\lambda)\left(H_{1}, H_{2}\right) \quad \text { in } \mathbb{R}_{+}^{N}, \\
(\nabla \psi) \cdot \mathbf{n}_{0}+(\nabla \psi) \cdot \mathbf{n}_{1} & =H_{2}+R_{2}(\lambda)\left(H_{1}, H_{2}\right) \quad \text { on } \mathbb{R}_{0}^{N},
\end{aligned}
$$

where

$$
\begin{aligned}
& R_{1}(\lambda)\left(H_{1}, H_{2}\right)=\gamma_{4 *}\left(A_{1} \nabla^{2} \mathcal{C}_{2}(\lambda) F_{\lambda}\left(H_{1}, H_{2}\right)+A_{2} \nabla \mathcal{C}_{2}(\lambda) F_{\lambda}\left(H_{1}, H_{2}\right)\right), \\
& R_{2}(\lambda)\left(H_{1}, H_{2}\right)=\left(\nabla \mathcal{C}_{2}(\lambda) F_{\lambda}\left(H_{1}, H_{2}\right)\right) \cdot \mathbf{n}_{1} .
\end{aligned}
$$

For $F=\left(F_{1}, F_{2}, F_{3}\right) \in \mathcal{Y}_{q}\left(\mathbb{R}_{+}^{N}\right)$, let

$$
\mathcal{R}_{1}(\lambda) F=\gamma_{4 *}\left(A_{1} \nabla^{2} \mathcal{C}_{2}(\lambda) F\right)+A_{2} \nabla \mathcal{C}_{2}(\lambda) F, \quad \mathcal{R}_{2}(\lambda) F=\left[\nabla \mathcal{C}_{2}(\lambda) F\right] \cdot \mathbf{n}_{1},
$$

and let $\mathcal{R}(\lambda) F=\left(\mathcal{R}_{1}(\lambda) F, \mathcal{R}_{2}(\lambda) F\right) \in Y_{q}\left(\mathbb{R}_{+}^{N}\right)$ and $R(\lambda)\left(H_{1}, H_{2}\right)=\left(R_{1}(\lambda)\left(H_{1}, H_{2}\right), R_{2}(\lambda)\left(H_{1}, H_{2}\right)\right)$. We then have

$$
\mathcal{R}(\lambda) F_{\lambda}\left(H_{1}, H_{2}\right)=R(\lambda)\left(H_{1}, H_{2}\right) .
$$

We now use the following two lemmas to calculate the $\mathcal{R}$-norm.

Lemma 19. (1) Let $X$ and $Y$ be Banach spaces, and let $\mathcal{T}$ and $\mathcal{S}$ be $\mathcal{R}$-bounded families in $\mathcal{L}(X, Y)$. Then, $\mathcal{T}+\mathcal{S}=\{T+S \mid T \in \mathcal{T}, S \in \mathcal{S}\}$ is also an $\mathcal{R}$-bounded family in $\mathcal{L}(X, Y)$ and

$$
\mathcal{R}_{\mathcal{L}(X, Y)}(\mathcal{T}+\mathcal{S}) \leq \mathcal{R}_{\mathcal{L}(X, Y)}(\mathcal{T})+\mathcal{R}_{\mathcal{L}(X, Y)}(\mathcal{S})
$$

(2) Let $X, Y$ and $Z$ be Banach spaces, and let $\mathcal{T}$ and $\mathcal{S}$ be $\mathcal{R}$-bounded families in $\mathcal{L}(X, Y)$ and $\mathcal{L}(Y, Z)$, respectively. Then, $\mathcal{S} \mathcal{T}=\{S T \mid T \in \mathcal{T}, S \in \mathcal{S}\}$ is also an $\mathcal{R}$-bounded family in $\mathcal{L}(X, Z)$ and

$$
\mathcal{R}_{\mathcal{L}(X, Z)}(\mathcal{S T}) \leq \mathcal{R}_{\mathcal{L}(X, Y)}(\mathcal{T}) \mathcal{R}_{\mathcal{L}(Y, Z)}(\mathcal{S})
$$

Lemma 20. Let $1<p, q<\infty$ and let $D$ be a domain in $\mathbb{R}^{N}$.

(1) Let $m(\lambda)$ be a bounded function defined on a subset $\Lambda$ in a complex plane $\mathbb{C}$ and let $M_{m}(\lambda)$ be a multiplication operator with $m(\lambda)$ defined by $M_{m}(\lambda) f=m(\lambda) f$ for any $f \in L_{q}(D)$. Then,

$$
\mathcal{R}_{\mathcal{L}\left(L_{q}(D)\right)}\left(\left\{M_{m}(\lambda) \mid \lambda \in \Lambda\right\}\right) \leq C_{N, q, D}\|m\|_{L_{\infty}(\Lambda)} .
$$

(2) Let $n(\tau)$ be a $C^{1}$ function defined on $\mathbb{R} \backslash\{0\}$ that satisfies the conditions: $|n(\tau)| \leq \gamma$ and $\left|\tau n^{\prime}(\tau)\right| \leq \gamma$ with some constant $\gamma>0$ for any $\tau \in \mathbb{R} \backslash\{0\}$. Let $T_{n}$ be an operator valued Fourier multiplier defined by $T_{n} f=\mathcal{F}^{-1}[n \mathcal{F}[f]]$ for any $f \in \mathcal{S}(\mathbb{R}, X)$ with $\mathcal{F}[f] \in \mathcal{D}(\mathbb{R}, X)$. Then, $T_{n}$ is extended to a bounded linear operator from $L_{p}\left(\mathbb{R}, L_{q}(D)\right)$ into itself. Moreover, denoting this extension also by $T_{n}$, we have

$$
\left\|T_{n}\right\|_{\mathcal{L}\left(L_{p}\left(\mathbb{R}, L_{q}(D)\right)\right)} \leq C_{p, q, D} \gamma .
$$

Remark 21. For proofs of Lemma 19 and Lemma 20, we refer to [10, p.28, 3.4.Proposition and p.27, 3.2.Remarks (4)] (cf. also Bourgain [6]), respectively. 
By Lemma 19, Lemma 20, (7.20), and Theorem 17 (2) we have

$$
\mathcal{R}_{\mathcal{L}\left(\mathcal{Y}_{q}\left(\mathbb{R}_{+}^{N}\right)\right)}\left(\left\{\left(\tau \partial_{\tau}\right)^{\ell} F_{\lambda} \mathcal{R}(\lambda) \mid \lambda \in \Sigma_{\epsilon, \lambda_{1}}\right\}\right) \leq r_{b}\left(C M_{1}+C_{M_{2}} \tilde{\lambda}_{0}^{-1 / 2}\right)
$$

for any $\tilde{\lambda}_{0} \geq \lambda_{0}$. In fact, by (7.20), we have

$$
\int_{0}^{1}\left\|\sum_{j-1}^{n} r_{j}(u) A_{2} \nabla \mathcal{C}_{2}\left(\lambda_{j}\right) F_{j}\right\|_{L_{q}\left(\mathbb{R}_{+}^{N}\right)}^{q} d u \leq C_{M_{2}}^{q} \int_{0}^{1}\left\|\sum_{j=1}^{n} r_{j}(u) \nabla \mathcal{C}_{2}\left(\lambda_{j}\right) F_{j}\right\|_{L_{q}\left(\mathbb{R}_{+}^{N}\right)}^{q} d u .
$$

By Lemma 20, we have

$$
\begin{aligned}
\int_{0}^{1}\left\|\sum_{j=1}^{n} r_{j}(u) \nabla \mathcal{C}_{2}\left(\lambda_{j}\right) F_{j}\right\|_{L_{q}\left(\mathbb{R}_{+}^{N}\right)}^{q} d u & =\int_{0}^{1}\left\|\sum_{j=1}^{n} r_{j}(u) \lambda_{j}^{-1 / 2} \lambda_{j}^{1 / 2} \nabla \mathcal{C}_{2}\left(\lambda_{j}\right) F_{j}\right\|_{L_{q}\left(\mathbb{R}_{+}^{N}\right)}^{q} d u \\
& \leq \tilde{\lambda}_{0}^{-q / 2} \int_{0}^{1}\left\|\sum_{j=1}^{n} r_{j}(u) \lambda_{j}^{1 / 2} \nabla \mathcal{C}_{2}\left(\lambda_{j}\right) F_{j}\right\|_{L_{q}\left(\mathbb{R}_{+}^{N}\right)}^{q} d u
\end{aligned}
$$

for any $\lambda_{j} \in \Sigma_{\epsilon, \tilde{\lambda}_{0}}$ and $\tilde{\lambda}_{0} \geq \lambda_{0}$. Thus, by Theorem[15(2), we have

$$
\int_{0}^{1}\left\|\sum_{j-1}^{n} r_{j}(u) A_{2} \nabla \mathcal{C}_{2}\left(\lambda_{j}\right) F_{j}\right\|_{L_{q}\left(\mathbb{R}_{+}^{N}\right)}^{q} d u \leq \tilde{\lambda}_{0}^{-q / 2} r_{b}^{q} \int_{0}^{1}\left\|\sum_{j=1}^{n} r_{j}(u) F_{j}\right\|_{L_{q}\left(\mathbb{R}_{+}^{N}\right)}^{q} d u .
$$

Analogously, we can estimate $\mathcal{R}_{\mathcal{L}\left(\mathcal{Y}_{q}\left(\mathbb{R}_{+}^{N}\right), L_{q}\left(\mathbb{R}_{+}^{N}\right)\right)}$ norm of $B_{1}$ and $B_{2}$ and $\mathcal{R}_{\mathcal{L}\left(\mathcal{Y}_{q}\left(\mathbb{R}_{+}^{N}\right), H_{q}^{1}\left(\mathbb{R}_{+}^{N}\right)\right)}$ norm of $B_{3}$, where $B_{1}=A_{1} \nabla^{2} \mathcal{C}_{2}(\lambda) F, B_{2}=\lambda^{1 / 2}\left[\nabla \mathcal{C}_{2}(\lambda) F\right] \cdot \mathbf{n}, B_{3}=\left[\nabla \mathcal{C}_{2}(\lambda) F\right] \cdot \mathbf{n}$, and so we have (7.24).

Choosing $M_{1}$ so small that $r_{b} C M_{1} \leq 1 / 4$ and choosing $\tilde{\lambda}_{0}$ so large that $r_{b} C_{M_{2}} \tilde{\lambda}_{0}^{-1 / 2} \leq 1 / 4$ in (7.24), we have

$$
\mathcal{R}_{\mathcal{L}\left(\mathcal{Y}_{q}\left(\mathbb{R}_{+}^{N}\right)\right)}\left(\left\{\left(\tau \partial_{\tau}\right)^{\ell} F_{\lambda} \mathcal{R}(\lambda) \mid \lambda \in \Sigma_{\epsilon, \tilde{\lambda}_{0}}\right\}\right) \leq 1 / 2 .
$$

Since $\mathcal{R}$-boundedness implies the usual boundedness, we have

$$
\left\|F_{\lambda} \mathcal{R}(\lambda) F_{\lambda}\left(H_{1}, H_{2}\right)\right\|_{\mathcal{Y}_{q}\left(\mathbb{R}_{+}^{N}\right)} \leq(1 / 2)\left\|F_{\lambda}\left(H_{1}, H_{2}\right)\right\|_{L_{q}\left(\mathcal{Y}_{q}\left(\mathbb{R}_{+}^{N}\right)\right.} .
$$

Here and in the following, the norm of $\mathcal{Y}_{q}\left(\mathbb{R}_{+}^{N}\right)$ is given by

$$
\left\|\left(F_{1}, F_{2}, F_{3}\right)\right\|_{\mathcal{Y}_{q}\left(\mathbb{R}_{+}^{N}\right)}=\left\|\left(F_{1}, F_{2}\right)\right\|_{L_{q}\left(\mathbb{R}_{+}^{N}\right)}+\left\|F_{3}\right\|_{H_{q}^{1}\left(\mathbb{R}_{+}^{N}\right)} .
$$

Thus, $\left\|F_{\lambda}\left(H_{1}, H_{2}\right)\right\|_{\mathcal{Y}_{q}\left(\mathbb{R}_{+}^{N}\right)}$ gives the equivalent norm of $Y_{q}\left(\mathbb{R}_{+}^{N}\right)$. By (7.23) and (7.25) we see that $(\mathbf{I}+R(\lambda))^{-1}=\sum_{j=0}^{\infty}(-R(\lambda))^{j}$ exists as an operator from $Y_{q}\left(\mathbb{R}_{+}^{N}\right)$ into itself and its operator norm does not exceed 2. Thus, in view of (7.21), $\varphi=\mathcal{C}_{2}(\lambda) F_{\lambda}(\mathbf{I}+R(\lambda))^{-1}\left(H_{1}, H_{2}\right)$ is a solution of Eq. (7.19).

On the other hand, by (7.25) and lemma 19, we see that $\left(\mathbf{I}+F_{\lambda} \mathcal{R}(\lambda)\right)^{-1}=\sum_{j=1}^{\infty}\left(F_{\lambda} \mathcal{R}(\lambda)\right)^{j}$ exists and

$$
\mathcal{R}_{\mathcal{L}\left(\mathcal{Y}_{q}\left(\mathbb{R}_{+}^{N}\right)\right)}\left(\left\{\left(\tau \partial_{\tau}\right)^{\ell}\left(\mathbf{I}+F_{\lambda} \mathcal{R}(\lambda)\right)^{-1} \mid \lambda \in \Sigma_{\epsilon, \tilde{\lambda}_{0}}\right\}\right) \leq 4
$$

Set $\tilde{\mathcal{C}}_{3}(\lambda)=\mathcal{C}_{2}(\lambda)\left(\mathbf{I}+F_{\lambda} \mathcal{R}(\lambda)\right)^{-1}$. Since $\mathcal{R}(\lambda) F_{\lambda}=R(\lambda)$ as follows from (7.23), we have

$$
\begin{aligned}
\left(\mathbf{I}+F_{\lambda} \mathcal{R}(\lambda)\right)^{-1} F_{\lambda} & =\sum_{j=1}^{\infty}(-1)^{j}\left(F_{\lambda} \mathcal{R}(\lambda)\right)^{j} F_{\lambda}=\sum_{j=0}^{\infty}(-1)^{j} F_{\lambda}\left(\mathcal{R}(\lambda) F_{\lambda}\right)^{j} \\
& =F_{\lambda} \sum_{j=0}^{\infty}(-R(\lambda))^{j}=F_{\lambda}(\mathbf{I}+R(\lambda))^{-1},
\end{aligned}
$$

which leads to $\varphi=\tilde{\mathcal{C}}_{3}(\lambda) F_{\lambda}\left(H_{1}, H_{2}\right)$. Thus, $\tilde{\mathcal{C}}_{3}(\lambda)$ is an $\mathcal{R}$-bounded solution operator for Eq. (7.19). Set

$$
\mathcal{C}_{3}(\lambda) F=\left[\tilde{\mathcal{C}}_{3}(\lambda)\left(F \circ \Phi^{-1}\right)\right] \circ \Phi,
$$

and then $\mathcal{C}_{3}(\lambda)$ is an $\mathcal{R}$-bounded solution operator of Eq. (7.17). This completes the proof of the assertion (2). 


\subsection{Proof of Theorem 15}

To prove Theorem 15, we need to use several properties of uniform $C^{2}$ domain, which are stated in the following proposition.

Proposition 22. Let $\Omega$ be a uniform $C^{2}$-domain in $\mathbb{R}^{N}$ with boundary $\Gamma$. Then, for any positive constant $M_{1}$, there exist constants $M_{2}>0, d \in(0,1)$, at most countably many functions $\Phi_{j} \in C^{2}\left(\mathbb{R}^{N}\right)$, and points $x_{j}^{1} \in \Omega$ and $x_{j}^{2} \in \Gamma(j \in \mathbb{N})$ such that the following assertions hold:

(1) For every $j \in \mathbb{N}$, the map $\mathbb{R}^{N} \ni x \rightarrow \Phi_{j}(x) \in \mathbb{R}^{N}$ is bijective.

(2) $\Omega=\left(\bigcup_{j=1}^{\infty} B_{d}\left(x_{j}^{1}\right)\right) \cup\left(\bigcup_{j=1}^{\infty}\left(\Phi_{j}\left(\mathbb{R}_{+}^{N}\right) \cap B_{d}\left(x_{j}^{2}\right)\right)\right), B_{d}\left(x_{j}^{1}\right) \subset \Omega, \Phi_{j}\left(\mathbb{R}_{+}^{N}\right) \cap B_{d}\left(x_{j}^{2}\right)=\Omega \cap B_{d}\left(x_{j}^{2}\right)$, and $\Phi_{j}\left(\mathbb{R}_{0}^{N}\right) \cap B_{d}\left(x_{j}^{2}\right)=\Gamma \cap B_{d}\left(x_{j}^{2}\right)$.

(3) There exist $C^{\infty}$ functions $\zeta_{j}^{i}(i=1,2, j \in \mathbb{N})$ such that $\operatorname{supp} \zeta_{j}^{i}, \operatorname{supp} \tilde{\zeta}_{j}^{i} \subset B_{d}\left(x_{j}^{i}\right),\left\|\zeta_{j}^{i}\right\|_{H_{\infty}^{2}\left(\mathbb{R}^{N}\right)} \leq c_{0}$, $\left\|\tilde{\zeta}_{j}^{i}\right\|_{H_{\infty}^{2}\left(\mathbb{R}^{N}\right)} \leq c_{0}, \tilde{\zeta}_{j}^{i}=1$ on $\operatorname{supp} \zeta_{j}^{i}, \sum_{i=1,2} \sum_{j=1}^{\infty} \zeta_{j}^{i}=1$ on $\bar{\Omega}, \sum_{j=1}^{\infty} \zeta_{j}^{1}=1$ on $\Gamma$. Here, $c_{0}$ is a constant which depends on $M_{2}, N, q, q^{\prime}$ and $r$, but is independent of $j \in \mathbb{N}$.

(4) $\nabla \Phi_{j}=\mathcal{R}_{j}+R_{i}, \nabla\left(\Phi_{j}\right)^{-}=\mathcal{R}_{j}^{-}+R_{j}^{-}$, where $\mathcal{R}_{j}$ and $\mathcal{R}_{j}^{-}$are $N \times N$ constant orthogonal matrices, and $R_{j}$ and $R_{j}^{-}$are $N \times N$ matrices of $H_{\infty}^{1}$ functions defined on $\mathbb{R}^{N}$ which satisfies the conditions: $\left\|R_{j}\right\|_{L_{\infty}\left(\mathbb{R}^{N}\right)} \leq M_{1},\left\|R_{j}^{-}\right\|_{L_{\infty}\left(\mathbb{R}^{N}\right)} \leq M_{1},\left\|\nabla R_{j}\right\|_{L_{\infty}\left(\mathbb{R}^{N}\right)} \leq M_{2}$ and $\left\|\nabla R_{j}^{-}\right\|_{L_{\infty}\left(\mathbb{R}^{N}\right)} \leq M_{2}$ for any $j \mathbb{N}$.

(5) There exist a natural number $L>2$ such that any $L+1$ distinct sets of $\left\{B_{d}\left(x_{j}^{i}\right) \mid i=1,2, j \in \mathbb{N}\right\}$ have an empty intersection.

In what follows, we write $\Omega_{\ell}=\Phi_{\ell}\left(\mathbb{R}_{+}^{N}\right)$ and $\Gamma_{\ell}=\Phi_{\ell}\left(\mathbb{R}_{0}^{N}\right)$ for $\ell \in \mathbb{N}$. Moreover, we write $B_{d}\left(x_{j}^{i}\right)$ simply by $B_{j}^{i}$. Since $\rho_{0}(x)$ and $\gamma_{k}(x)(k=1,3,4)$ are uniformly continuous functions on $\bar{\Omega}$, choosing $d$ smaller if necessary, we may assume that

$$
\left|\rho_{0}(x)-\rho_{0}\left(x_{j}^{i}\right)\right| \leq M_{1}, \quad\left|\gamma_{k}(x)-\gamma_{k}\left(x_{j}^{i}\right)\right| \leq M_{1} \quad \text { for } x \in B_{J}^{i} \cap \bar{\Omega}, \quad k=1,3,4 .
$$

By the finite intersection property stated in Proposition 22 (5), we have

$$
\left(\sum_{i=1,2} \sum_{j=1}^{\infty}\|f\|_{L_{q}\left(B_{j}^{i} \cap \Omega\right)}^{q}\right)^{1 / q} \leq C_{q}\|f\|_{L_{q}(\Omega)}
$$

for any $f \in L_{q}(\Omega)$ and $1 \leq q<\infty$. In particular, by (7.28) we have

Lemma 23. Let $i=1,2$ and $1<q<\infty$. Let $\left\{f_{j}\right\}_{j=0}^{\infty}$ be a sequence of functions in $L_{q}(\Omega)$ such that $\sum_{j=0}^{\infty}\left\|f_{j}\right\|_{L_{q}(\Omega)}^{q}<\infty$, and $\operatorname{supp} f_{j} \subset B_{j}^{i}(j \in \mathbb{N})$. Then, $\sum_{j=0}^{\infty} f_{j} \in L_{q}(\Omega)$ and $\left\|\sum_{j=1}^{\infty} f_{j}\right\|_{L_{q}(\Omega)} \leq$ $\left(\sum_{j=1}^{\infty}\left\|f_{j}\right\|_{L_{q}(\Omega)}^{q}\right)^{1 / q}$.

We first prove the assertion (1) in Theorem 15. We construct a parametrix. Let $\mathbf{v}_{j}^{1} \in H_{q}^{2}\left(\mathbb{R}^{N}\right)^{N}$ be solutions of the equations:

$$
\rho_{0}\left(x_{j}^{1}\right) \lambda \mathbf{v}_{j}^{1}-\mu \Delta \mathbf{v}_{j}^{1}-\nu \nabla \operatorname{div} \mathbf{v}_{j}^{1}-\gamma_{1}\left(x_{j}^{1}\right) \rho_{0}\left(x_{j}^{1}\right) \lambda^{-1} \nabla \operatorname{div} \mathbf{v}_{j}^{1}=\zeta_{j}^{1} \mathbf{g} \quad \text { in } \mathbb{R}^{N}
$$

and $\mathbf{v}_{j}^{2} \in H_{q}^{2}\left(\Omega_{j}\right)^{N}$ solutions of the equations:

$$
\rho_{0}\left(x_{j}^{2}\right) \lambda \mathbf{v}_{j}^{2}-\mu \Delta \mathbf{v}_{j}^{2}-\nu \nabla \operatorname{div} \mathbf{v}_{j}^{2}-\gamma_{1}\left(x_{j}^{2}\right) \rho_{0}\left(x_{j}^{2}\right) \lambda^{-1} \nabla \operatorname{div} \mathbf{v}_{j}^{2}=\zeta_{j}^{2} \mathbf{g} \quad \text { in } \Omega_{j},\left.\quad \mathbf{v}_{j}^{2}\right|_{\Gamma_{j}}=0 .
$$

By Theorem 16 (1) and Theorem 18(1), there are $\mathcal{R}$-bounded solution operators $\mathcal{C}_{j}^{i}(\lambda)$ with

$$
\mathcal{C}_{j}^{i}(\lambda) \in \operatorname{Hol}\left(\Sigma_{\epsilon, \lambda_{0}}, \mathcal{L}\left(L_{q}\left(\Omega_{j}^{i}\right)^{N}, H_{q}^{2}\left(\Omega_{j}^{i}\right)^{N}\right)\right)
$$


such that for any $\mathbf{g} \in L_{q}(\Omega)$ and $\lambda \in \Sigma_{\epsilon, \lambda_{0}}, \mathbf{v}_{j}^{1}=\mathcal{C}_{j}^{1}(\lambda) \zeta_{j}^{1} \mathbf{g}$ are solutions of Eq. (7.29) and $\mathbf{v}_{j}^{2}=\mathcal{C}_{j}^{2}(\lambda) \zeta_{j}^{2} \mathbf{g}$ solutions of Eq. (7.30), where we have set $\Omega_{j}^{1}=\mathbb{R}^{N}$ and $\Omega_{j}^{2}=\Omega_{j}$. Moreover, we have

$$
\mathcal{R}_{\mathcal{L}\left(L_{q}\left(\Omega_{j}^{i}\right)^{N}, H_{q}^{2-k}\left(\Omega_{j}^{i}\right)^{N}\right)}\left(\left\{\left(\tau \partial_{\tau}\right)^{\ell}\left(\lambda^{k / 2} \mathcal{C}_{j}^{i}(\lambda)\right) \mid \lambda \in \Sigma_{\epsilon, \lambda_{0}}\right\}\right) \leq r_{b}
$$

for $\ell=0,1$ and $k=0,1,2$. Notice that $\lambda_{0}$ and $r_{b}$ are independent of $i=1,2$ and $j \in \mathbb{N}$. Let

$$
\mathcal{U}_{1}(\lambda) \mathbf{g}=\sum_{i=1,2} \sum_{j=1}^{\infty} \tilde{\zeta}_{j}^{i} \mathcal{C}_{j}^{i}(\lambda) \zeta_{j}^{i} \mathbf{g}
$$

for $\mathbf{g} \in L_{q}(\Omega)^{N}$. By Lemma 23, we have

$$
\mathcal{R}_{\mathcal{L}\left(L_{q}(\Omega)^{N}, H_{q}^{2-k}(\Omega)^{N}\right)}\left(\left\{\left(\tau \partial_{\tau}\right)^{\ell}\left(\lambda^{k / 2} \mathcal{U}(\lambda)\right) \mid \lambda \in \Sigma_{\epsilon, \lambda_{0}}\right\}\right) \leq C_{N, q} r_{b} .
$$

In fact, by (7.31) and (7.28) we have

$$
\begin{aligned}
& \sum_{i=1,2} \sum_{j=1}^{\infty} \int_{0}^{1} \int_{\Omega}\left|\sum_{k=1}^{n} r_{k}(u) \tilde{\zeta}_{j}^{i} \mathcal{C}_{j}^{i}\left(\lambda_{k}\right) \zeta_{j}^{i} \mathbf{g}_{k}\right|^{q} d x d u \leq c_{0}^{q} \sum_{i=1,2} \sum_{j=1}^{\infty} \int_{0}^{1} \int_{\Omega_{j}^{i}}\left|\sum_{k=1}^{n} r_{k}(u) \mathcal{C}_{j}^{i}\left(\lambda_{k}\right) \zeta_{j}^{i} \mathbf{g}_{k}\right|^{q} d x d u \\
& \leq\left(c_{0} r_{b}\right)^{q} \sum_{i=1,2} \sum_{j=1}^{\infty} \int_{0}^{1} \int_{\Omega_{j}^{i}}\left|\sum_{k=1}^{n} r_{k}(u) \zeta_{j}^{i} \mathbf{g}_{k}\right|^{q} d x d u \leq\left(c_{0}^{2} r_{b}\right)^{q} \sum_{i=0,1} \sum_{j=1}^{\infty} \int_{0}^{1} \int_{\Omega \cap B_{j}^{i}}\left|\sum_{k=1}^{n} r_{k}(u) \mathbf{g}_{k}\right|^{q} d x d u \\
& =\left(c_{0}^{2} r_{b}\right)^{q} \int_{0}^{1}\left(\sum_{i=0,1} \sum_{j-1}^{\infty} \int_{\Omega \cap B_{j}^{i}}\left|\sum_{k=1}^{n} r_{k}(u) \mathbf{g}_{k}\right|^{q} d x\right) d u \leq\left(C_{q} c_{0}^{2} r_{b}\right)^{q} \int_{0}^{1}\left\|\sum_{k=1}^{n} r_{k}(u) \mathbf{g}_{k}\right\|_{L_{q}(\Omega)}^{q} d u,
\end{aligned}
$$

and so by Lemma 23 we have

$$
\left\|\sum_{k=1}^{n} r_{k}(u) \mathcal{U}_{1}\left(\lambda_{k}\right) \mathbf{g}_{k}\right\|_{L_{q}(\Omega \times(0,1))} \leq C_{q} c_{0}^{2} r_{b}\left\|\sum_{k=1}^{n} r_{k}(u) \mathbf{g}_{k}\right\|_{L_{q}(\Omega \times(0,1))} .
$$

In this way, we can show (7.32). Next, since

$$
\begin{aligned}
\Delta\left(\tilde{\zeta}_{j}^{i} \mathbf{v}_{j}^{i}\right) & =\tilde{\zeta}_{j}^{i} \Delta \mathbf{v}_{j}^{i}+2\left(\nabla \tilde{\zeta}_{j}^{i}\right) \nabla \mathbf{v}_{j}^{i}+\left(\Delta \tilde{\zeta}_{j}^{i}\right) \mathbf{v}_{j}^{i}, \\
\nabla \operatorname{div}\left(\tilde{\zeta}_{j}^{i} \mathbf{v}_{j}^{i}\right) & =\tilde{\zeta}_{j}^{i} \nabla \operatorname{div} \mathbf{v}_{j}^{i}+\left(\nabla \zeta_{j}^{i}\right) \operatorname{div} \mathbf{v}_{j}^{i}+\nabla\left(\left(\nabla \zeta_{j}^{i}\right) \cdot \mathbf{v}_{j}^{i}\right), \\
\nabla\left(\rho_{0}(x) \operatorname{div}\left(\tilde{\zeta}_{j}^{i} \mathbf{v}_{j}^{i}\right)\right) & =\tilde{\zeta}_{j}^{i} \rho_{0}(x) \nabla \operatorname{div} \mathbf{v}_{j}^{i}+\nabla\left(\rho_{0}(x)\left(\nabla \zeta_{j}^{i}\right) \cdot \mathbf{v}_{j}^{i}\right)+\left(\nabla \zeta_{j}^{i}\right) \rho_{0}(x) \operatorname{div} \mathbf{v}_{j}^{i},
\end{aligned}
$$

with $\mathbf{v}_{j}^{i}=\mathcal{C}_{j}^{i}(\lambda) \zeta_{j}^{i} \mathbf{g}$, setting

$$
\begin{aligned}
& \mathcal{V}_{1}(\lambda) \mathbf{g}=-\sum_{i=1,2} \sum_{j=1}^{\infty}\left\{\mu \left(2\left(\nabla \tilde{\zeta}_{j}^{i}\right) \nabla \mathcal{C}_{j}^{i}(\lambda) \zeta_{j}^{i} \mathbf{g}+\left(\Delta \tilde{\zeta}_{j}^{i}\right) \mathcal{C}_{j}^{i}(\lambda) \zeta_{j}^{i} \mathbf{g}+\nu\left(\left(\nabla \zeta_{j}^{i}\right) \operatorname{div} \mathcal{C}_{j}^{i}(\lambda) \zeta_{j}^{i} \mathbf{g}+\nabla\left(\left(\nabla \zeta_{j}^{i}\right) \mathcal{C}_{j}^{i}(\lambda) \zeta_{j}^{i} \mathbf{g}\right)\right.\right.\right. \\
& \left.\quad+\lambda^{-1} \gamma_{1}(x)\left(\nabla\left(\rho_{0}(x)\left(\nabla \zeta_{j}^{i}\right) \cdot \mathcal{C}_{j}^{i}(\lambda) \zeta_{j}^{i} \mathbf{g}\right)+\left(\nabla \zeta_{j}^{i}\right) \rho_{0}(x) \operatorname{div} \mathcal{C}_{j}^{i}(\lambda) \zeta_{j}^{i} \mathbf{g}\right)\right\} \\
& \quad+\sum_{i=1,2} \sum_{j=1}^{\infty} \tilde{\zeta}_{j}^{i}\left(\left(\rho_{0}(x)-\rho_{0}\left(x_{j}^{i}\right)\right) \lambda \mathcal{C}_{j}^{i}(\lambda) \zeta_{j}^{i} \mathbf{g}-\left(\gamma_{1}(x) \rho_{0}(x)-\gamma_{1}\left(x_{j}^{i}\right) \rho_{0}\left(x_{j}^{i}\right)\right) \lambda^{-1} \nabla \mathcal{C}_{j}^{i}(\lambda) \zeta_{j}^{i} \mathbf{g}\right)
\end{aligned}
$$

and setting $\mathbf{v}=\mathcal{U}_{1}(\lambda) \mathbf{g}$, we have

$$
\rho_{0}(x) \lambda \mathbf{v}-\mu \Delta \mathbf{v}-\nu \nabla \operatorname{div} \mathbf{v}-\gamma_{1}(x) \lambda^{-1} \nabla\left(\rho_{0}(x) \operatorname{div} \mathbf{v}\right)=\mathbf{g}+\mathcal{V}_{1}(\lambda) \mathbf{g} \quad \text { in } \Omega,\left.\quad \mathbf{v}\right|_{\Gamma}=0
$$

because $\Gamma \cap B_{j}^{1}=\Gamma_{j}$ and $\tilde{\zeta}_{j}^{i} \zeta_{j}^{i}=\zeta_{j}^{i}$. Using (5.2), (7.27), Lemma 23) (7.31), and (7.32), we obtain

$$
\mathcal{R}_{\mathcal{L}\left(L_{q}(\Omega)^{N}\right)}\left(\left\{\left(\tau \partial_{\tau}\right)^{\ell} \mathcal{R}(\lambda) \mid \lambda \in \Sigma_{\epsilon, \tilde{\lambda}_{0}}\right\}\right) \leq c_{0}^{2} C_{q}\left\{\left(1+2 \alpha_{4}\right) M_{1}+\alpha_{4} r_{b} \tilde{\lambda}_{0}^{-1 / 2}\right\}
$$

for any $\tilde{\lambda}_{0} \geq \lambda_{0}$, where we have assumed that $\tilde{\lambda}_{0} \geq 1$. To prove (7.35), we have to estimate $\left(\nabla \rho_{0}\right)\left(\nabla \zeta_{j}^{i}\right)$. $\mathcal{C}_{j}^{i}(\lambda) \zeta_{j}^{i} \mathbf{g}$. For this purpose, we use the following lemma can be proved easily with the help of Sobolev's imbedding theorem. 
Lemma 24. Let $1<q \leq r<\infty$ and $N<r<\infty$. Then, the following two inequalities hold:

(1) There exists a constant $C$ depending only on $N, q$, and $r$ for which

$$
\|a b\|_{L_{q}(\Omega)} \leq C\|a\|_{L_{r}(\Omega)}\|b\|_{H_{q}^{1}(\Omega)} .
$$

(2) For any $\sigma>0$, there exists a constant $C=C_{\sigma,\|a\|_{L_{r}(\Omega)}}$ for which

$$
\|a b\|_{L_{q}(\Omega)} \leq \sigma\|b\|_{H_{q}^{1}(\Omega)}+C\|b\|_{L_{q}(\Omega)} .
$$

For any $\lambda_{k} \in \Sigma_{\epsilon, \tilde{\lambda}_{0}}$ and $\mathbf{g}_{k} \in L_{q}(\Omega)^{N}(k=1, \ldots, n)$, by Lemma 24, (7.31) and (5.2),

$$
\begin{aligned}
& \sum_{i=1,2} \sum_{j=1}^{\infty} \int_{0}^{1} \int_{\Omega}\left|\sum_{k=1}^{n} r_{k}(u)\left(\nabla \rho_{0}\right)\left(\nabla \zeta_{j}^{i}\right) \cdot \mathcal{C}_{j}^{i}\left(\lambda_{k}\right) \zeta_{j}^{i} \mathbf{g}_{k}\right|^{q} d x d u \\
& \leq\left(c_{0} \alpha_{4}\right)^{q} \sum_{i=1,2} \sum_{j=1}^{\infty} \int_{0}^{1}\left\|\sum_{k=1}^{n} r_{k}(u) \mathcal{C}_{j}^{i}\left(\lambda_{k}\right) \zeta_{j}^{i} \mathbf{g}_{k}\right\|_{H_{q}^{1}\left(\Omega_{j}^{i}\right)}^{q} d u \\
& \leq\left(c_{0} \alpha_{4}\right)^{q} \sum_{i=1,2} \sum_{j=1}^{\infty} \int_{0}^{1}\left\|\sum_{k=1}^{n} r_{k}(u) \lambda_{k}^{-1 / 2} \lambda_{k}^{1 / 2} \mathcal{C}_{j}^{i}\left(\lambda_{k}\right) \zeta_{j}^{i} \mathbf{g}_{k}\right\|_{H_{q}^{1}\left(\Omega_{j}^{i}\right)}^{q} d u \\
& \leq\left(c_{0} \alpha_{4} \tilde{\lambda}_{0}^{-1 / 2}\right)^{q} \sum_{i=1,2} \sum_{j=1}^{\infty} \int_{0}^{1}\left\|\sum_{k=1}^{n} r_{k}(u) \lambda_{k}^{1 / 2} \mathcal{C}_{j}^{i}\left(\lambda_{k}\right) \zeta_{j}^{i} \mathbf{g}_{k}\right\|_{H_{q}^{1}\left(\Omega_{j}^{i}\right)}^{q} d u \\
& \leq\left(c_{0} \alpha_{4} \tilde{\lambda}_{0}^{-1 / 2} r_{b}\right)^{q} \sum_{i=1,2} \sum_{j=1}^{\infty} \int_{0}^{1}\left\|\sum_{k=1}^{n} r_{k}(u) \zeta_{j}^{i} \mathbf{g}_{k}\right\|_{L_{q}\left(\Omega_{j}^{i}\right)}^{q} d u \\
& \leq\left(c_{0}^{2} \alpha_{4} \tilde{\lambda}_{0}^{-1 / 2} r_{b}\right)^{q} \sum_{i=1,2} \sum_{j=1}^{\infty} \int_{0}^{1}\left\|\sum_{k=1}^{n} r_{k}(u) \mathbf{g}_{k}\right\|_{L_{q}\left(\Omega \cap B_{j}^{i}\right)}^{q} d u \\
& =\left(c_{0}^{2} \alpha_{4} \tilde{\lambda}_{0}^{-1 / 2} r_{b}\right)^{q} \int_{0}^{1}\left(\sum_{i=1,2}^{\infty} \sum_{j=1}^{\infty}\left\|\sum_{k=1}^{n} r_{k}(u) \mathbf{g}_{k}\right\|_{L_{q}\left(\Omega \cap B_{j}^{i}\right)}^{q}\right) d u \\
& =\left(C_{q} c_{0}^{2} \alpha_{4} \tilde{\lambda}_{0}^{-1 / 2} r_{b}\right)^{q} \int_{0}^{1}\left\|\sum_{k=1}^{n} r_{k}(u) \mathbf{g}_{k}\right\|^{q} d u
\end{aligned}
$$

Other terms can be estimated similarly, and so by Lemma 23 and (7.28) we have (7.35). Choosing $M_{1}>0$ so small that $c_{0}^{2} C_{q}\left(1+2 \alpha_{4}\right) M_{1} \leq 1 / 4$ and choosing $\tilde{\lambda}_{0} \geq \max \left(\lambda_{0}, 1\right)$ so large that $c_{0}^{2} C_{q} \alpha_{4} r_{b} \tilde{\lambda}_{0}^{-1 / 2} \leq 1 / 4$, by (7.35) we have

$$
\mathcal{R}_{\mathcal{L}\left(L_{q}(\Omega)^{N}\right)}\left(\left\{\left(\tau \partial_{\tau}\right)^{\ell} \mathcal{V}_{1}(\lambda) \mid \lambda \in \Sigma_{\epsilon, \tilde{\lambda}_{0}}\right\}\right) \leq 1 / 2
$$

Thus, $\left(\mathbf{I}+\mathcal{V}_{1}(\lambda)\right)^{-1}=\sum_{j=1}^{\infty}\left(-\mathcal{V}_{1}(\lambda)\right)^{j}$ exists and satisfies the estimate:

$$
\mathcal{R}_{\mathcal{L}\left(L_{q}(\Omega)^{N}\right)}\left(\left\{\left(\tau \partial_{\tau}\right)^{\ell}\left(\mathbf{I}+\mathcal{V}_{1}(\lambda)\right)^{-1} \mid \lambda \in \Sigma_{\epsilon, \tilde{\lambda}_{0}}\right\}\right) \leq 4
$$

Let $\mathcal{C}(\lambda)=\mathcal{U}_{1}(\lambda)\left(\mathbf{I}+\mathcal{V}_{1}(\lambda)\right)^{-1}$, and then in view of (17.34) we see that $\mathbf{u}=\mathcal{C}(\lambda) \mathbf{g}$ is a solution of Eq. (7.3). The uniqueness of solutions follows from the existence of solutions of the dual problem. Moreover, by (7.32) and (7.36) we see that $\mathcal{C}(\lambda)$ satisfies the estimate:

$$
\mathcal{R}_{\mathcal{L}\left(L_{q}(\Omega)^{N}, H_{q}^{2-k}(\Omega)^{N}\right)}\left(\left\{\left(\tau \partial_{\tau}\right)^{\ell}\left(\lambda^{k / 2} \mathcal{C}(\lambda)\right) \mid \lambda \in \Sigma_{\epsilon, \lambda_{0}}\right\}\right) \leq 4 C_{N, q} r_{b}
$$

This completes the proof of assertion (1) of Theorem 15

We next prove the assertion (2). By Theorem 16 (2) and Theorem 18 (2), there are $\mathcal{R}$-bounded solution operators $\mathcal{D}_{j}^{i}(\lambda)$ with

$$
\mathcal{D}_{j}^{1}(\lambda) \in \operatorname{Hol}\left(\Sigma_{\epsilon, \lambda_{0}}, \mathcal{L}\left(L_{q}\left(\mathbb{R}^{N}\right), H_{q}^{2}\left(\mathbb{R}^{N}\right)\right)\right), \quad \mathcal{D}_{j}^{2}(\lambda) \in \operatorname{Hol}\left(\Sigma_{\epsilon, \lambda_{0}}, \mathcal{L}\left(\mathcal{Y}_{q}\left(\Omega_{1}\right), H_{q}^{2}\left(\mathbb{R}^{N}\right)\right)\right)
$$


such that for any $\left(h_{1}, h_{2}\right) \in Y_{q}(\Omega)$ and $\lambda \in \Sigma_{\epsilon, \lambda_{0}}, \vartheta_{j}^{1}=\mathcal{D}_{j}^{1}(\lambda) \zeta_{j}^{1} h_{1}$ are solutions of the equations:

$$
\gamma_{3}\left(x_{j}^{1}\right) \lambda \vartheta_{j}^{1}-\gamma_{4}\left(x_{j}^{1}\right) \Delta \vartheta_{j}^{1}=\zeta_{j}^{1} h_{1} \quad \text { in } \mathbb{R}^{N},
$$

and $\vartheta_{j}^{2}=\mathcal{D}_{j}^{2}(\lambda) \zeta_{j}^{2}\left(h_{1}, \lambda^{1 / 2} h_{2}, h_{2}\right)$ are solutions of the equations:

$$
\gamma_{3}\left(x_{j}^{2}\right) \lambda \vartheta_{j}^{2}-\gamma_{4}\left(x_{j}^{2}\right) \Delta \vartheta_{j}^{2}=\zeta_{j}^{2} h_{1} \quad \text { in } \Omega_{j},\left.\quad\left(\nabla \vartheta_{j}^{2}\right) \cdot \mathbf{n}_{j}\right|_{\Gamma_{j}}=0
$$

where $\mathbf{n}_{j}$ is the unit outer normal to $\Gamma_{j}$. Notice that $\mathbf{n}_{j}=\mathbf{n}$ on $\Gamma_{j} \cap B_{j}^{2}=\Gamma \cap B_{j}^{2}$. In particular, by (7.37) we have

$$
\sum_{k=0}^{2}|\lambda|^{k / 2}\left\|\vartheta_{j}^{i}\right\|_{H_{q}^{2-k}\left(\Omega_{j}^{i}\right.} \leq r_{b}\left\{\left\|\zeta_{j}^{i} h_{1}\right\|_{L_{q}\left(\Omega_{j}^{i}\right)}+\sigma^{i}\left(\left\|\lambda^{1 / 2} h_{2}\right\|_{L_{q}\left(\Omega_{j}^{2}\right)}+\left\|h_{2}\right\|_{H_{q}^{1}\left(\Omega_{j}^{2}\right)}\right)\right\} \quad(i=1,2),
$$

where $\sigma^{1}=0$ and $\sigma^{2}=1$. Let

$$
\mathbf{U}_{2}(\lambda)\left(h_{1}, h_{2}\right)=\sum_{i=1,2} \sum_{j=1}^{\infty} \tilde{\zeta}_{j}^{i} \vartheta_{j}^{i}, \quad \mathcal{U}_{2}(\lambda) F=\sum_{j=1}^{\infty} \tilde{\zeta}_{j}^{1} \mathcal{D}_{j}^{1}(\lambda) \zeta_{j}^{1} F_{1}+\sum_{j=1}^{\infty} \tilde{\zeta}_{j}^{2} \mathcal{D}_{j}^{2}(\lambda) \zeta_{j}^{2} F
$$

for $\left(h_{1}, h_{2}\right) \in Y_{q}(\Omega)$ and $F=\left(F_{1}, F_{2}, F_{3}\right) \in \mathcal{Y}_{q}(\Omega)$. By Lemma 23 and (7.28), we have

$$
\sum_{k=0}^{2}|\lambda|^{k / 2}\left\|\mathbf{U}_{2}(\lambda)\left(h_{1}, h_{2}\right)\right\|_{H_{q}^{2-k}(\Omega)} \leq C_{N, q} r_{b}\left(\left\|h_{1}\right\|_{L_{q}(\Omega)}+|\lambda|^{1 / 2}\left\|h_{2}\right\|_{L_{q}(\Omega)}+\left\|h_{2}\right\|_{H_{q}^{1}(\Omega)}\right)
$$

for any $\lambda \in \Sigma_{\epsilon, \lambda_{0}}$ and $\left(h_{1}, h_{2}\right) \in Y_{q}(\Omega)$, and

$$
\mathcal{R}_{\mathcal{L}\left(\mathcal{Y}_{q}(\Omega), H_{q}^{2-k}(\Omega)\right)}\left(\left\{\left(\tau \partial_{\tau}\right)^{\ell}\left(\lambda^{k / 2} \mathcal{U}_{2}(\lambda)\right) \mid \lambda \in \Sigma_{\epsilon, \lambda_{0}}\right\}\right) \leq C_{N, q} r_{b}
$$

for $k=0,1,2$ and $\ell=0,1$. For $F=\left(F_{1}, F_{2}, F_{3}\right) \in \mathcal{Y}_{q}(\Omega)$, let

$$
\begin{aligned}
\mathcal{V}_{21}(\lambda) F & =-\sum_{i=1,2} \sum_{j=1}^{\infty}\left\{\operatorname{div}\left(\gamma_{4}(x)\left(\nabla \zeta_{j}^{i}\right) \mathcal{D}_{j}^{i}(\lambda) \zeta_{j}^{i} F+\nabla\left(\gamma_{4} \zeta_{j}^{i}\right) \cdot \nabla\left(\mathcal{D}_{j}^{i}(\lambda) \zeta_{j}^{i} F\right)\right\}\right. \\
& +\sum_{i=1,2} \sum_{j=1}^{\infty} \zeta_{j}^{i}\left\{\left(\gamma_{3}(x)-\gamma_{3}\left(x_{j}^{i}\right)\right) \lambda \mathcal{D}_{j}^{i}(\lambda) \zeta_{j}^{i} F-\operatorname{div}\left(\left(\gamma_{4}(x)-\rho\left(x_{j}^{i}\right)\right) \nabla \mathcal{D}_{j}^{i}(\lambda) \zeta_{j}^{i} F\right)\right\}, \\
\mathcal{V}_{22}(\lambda) F & =-\sum_{j=1}^{\infty}\left(\nabla \zeta_{j}^{2}\right) \cdot \mathbf{n}_{j} \mathcal{D}_{j}^{2}(\lambda) \zeta_{j}^{i} F,
\end{aligned}
$$

where we have set $\mathcal{D}_{j}^{1}(\lambda) \zeta_{j}^{i} F=\mathcal{D}_{j}^{1}(\lambda) \zeta_{j}^{i} F_{1}$. We then have

$$
\begin{aligned}
& \gamma_{3}(x) \lambda \mathbf{U}_{2}(\lambda)\left(h_{1}, h_{2}\right)-\operatorname{div}\left(\gamma_{4}(x) \nabla \mathbf{U}_{2}(\lambda)\left(h_{1}, h_{2}\right)\right)=h_{1}+\mathcal{V}_{21}(\lambda) F_{\lambda}\left(h_{1}, h_{2}\right) \text { in } \Omega \text {, } \\
& \left(\nabla \mathbf{U}_{2}(\lambda)\left(h_{1}, h_{2}\right)\right) \cdot \mathbf{n}=h_{2}+\mathcal{V}_{22}(\lambda) F_{\lambda}\left(h_{1}, h_{2}\right) \quad \text { on } \Gamma
\end{aligned}
$$

for any $\left(h_{1}, h_{2}\right) \in Y_{q}(\Omega)$, where we have set $F_{\lambda}\left(h_{1}, h_{2}\right)=\left(h_{1}, \lambda^{1 / 2} h_{2}, h_{2}\right) \in \mathcal{Y}_{q}(\Omega)$. Since

$$
\left\|\left(\nabla \gamma_{4}\right) \cdot \nabla \mathcal{D}_{j}^{i}(\lambda) \zeta_{j}^{i} F\right\|_{L_{q}(\Omega)} \leq \sigma\left\|\nabla \mathcal{D}_{j}^{i}(\lambda) \zeta_{j}^{i} F\right\|_{H_{q}^{1}(\Omega)}+C_{\sigma, \alpha_{4}}\left\|\nabla \mathcal{D}_{j}^{i}(\lambda) \zeta_{j}^{i} F\right\|_{L_{q}(\Omega)}
$$

as follows from Lemma 24 (2), by (7.37), Lemma 23, (7.27), (7.28) and Lemma 24, we have

$$
\mathcal{R}_{\mathcal{L}\left(\mathcal{Y}_{q}(\Omega)\right)}\left(\left\{\left(\tau \partial_{\tau}\right)^{\ell}\left(F_{\lambda}\left(\mathcal{V}_{21}(\lambda), \mathcal{V}_{22}(\lambda)\right)\right) \mid \lambda \in \Sigma_{\epsilon, \tilde{\lambda}_{0}}\right\}\right) \leq\left\{2 M_{1}+\sigma+c_{0}^{2} C_{q} C_{\sigma, \alpha_{4}} \tilde{\lambda}_{0}^{-1 / 2}\right\} r_{b}
$$

for any $\tilde{\lambda}_{0} \geq \max \left(\lambda_{0}, 1\right)$. Choosing $M_{1}$ and $\sigma>0$ so small that $2 M_{1} r_{b}<1 / 8, \sigma r_{b}<1 / 8$ and choosing $\tilde{\lambda}_{0}$ so large that $c_{0}^{2} C_{q} C_{\sigma, \alpha_{4}} r_{b} \tilde{\lambda}_{0}^{-1 / 2} \leq 1 / 4$, we have

$$
\mathcal{R}_{\mathcal{L}\left(\mathcal{Y}_{q}(\Omega)\right)}\left(\left\{\left(\tau \partial_{\tau}\right)^{\ell}\left(F_{\lambda}\left(\mathcal{V}_{21}(\lambda), \mathcal{V}_{22}(\lambda)\right)\right) \mid \lambda \in \Sigma_{\epsilon, \tilde{\lambda}_{0}}\right\}\right) \leq 1 / 2
$$


and so $\left(\mathbf{I}+F_{\lambda}\left(\mathcal{V}_{21}(\lambda), \mathcal{V}_{22}(\lambda)\right)\right)^{-1}=\sum_{j=0}^{\infty}\left(-F_{\lambda}\left(\mathcal{V}_{21}(\lambda), \mathcal{V}_{22}(\lambda)\right)\right)^{j}$ exists and

$$
\mathcal{R}_{\mathcal{L}\left(\mathcal{Y}_{q}(\Omega)\right)}\left(\left\{\left(\tau \partial_{\tau}\right)^{\ell}\left(\mathbf{I}+F_{\lambda}\left(\mathcal{V}_{21}(\lambda), \mathcal{V}_{22}(\lambda)\right)\right)^{-1} \mid \lambda \in \Sigma_{\epsilon, \tilde{\lambda}_{0}}\right\}\right) \leq 4
$$

On the other hand, by (7.44) we have

$$
\left\|F_{\lambda}\left(\mathcal{V}_{21}(\lambda) F_{\lambda}\left(h_{1}, h_{2}\right), \mathcal{V}_{22}(\lambda) F_{\lambda}\left(h_{1}, h_{2}\right)\right)\right\|_{\mathcal{Y}_{q}(\Omega)} \leq(1 / 2)\left\|F_{\lambda}\left(h_{1}, h_{2}\right)\right\|_{L_{q}(\Omega)}
$$

for any $\lambda \in \Sigma_{\epsilon, \tilde{\lambda}_{0}}$. Since $\left\|F_{\lambda}\left(h_{1}, h_{2}\right)\right\|_{\mathcal{Y}_{q}(\Omega)}=\left\|h_{1}\right\|_{L_{q}(\Omega)}+|\lambda|^{1 / 2}\left\|h_{2}\right\|_{L_{q}(\Omega)}+\left\|h_{2}\right\|_{H_{q}^{1}(\Omega)}$ gives equivalent norms in $Y_{q}(\Omega)$, we see that for each $\left.\lambda \in \Sigma_{\epsilon, \tilde{\lambda}_{0}}, \mathbf{I}+\left(\mathcal{V}_{21}(\lambda), \mathcal{V}_{22}(\lambda)\right) F_{\lambda}\right)^{-1}=\sum_{j=0}^{\infty}\left(-\left(\mathcal{V}_{21}(\lambda), \mathcal{V}_{22}(\lambda)\right) F_{\lambda}\right)^{j}$ exists as an operator in $\mathcal{L}\left(Y_{q}(\Omega)\right)$ whose operator norm does not exceed 2. Thus, in view of (7.43), $\vartheta=\mathbf{U}_{2}(\lambda)\left(\mathbf{I}+\left(\mathcal{V}_{21}(\lambda), \mathcal{V}_{22}(\lambda)\right)\right)^{-1}\left(h_{1}, h_{2}\right)$ is a solution of Eq. (7.4). The uniqueness of solution follows from the existence of solutions for the dual problem. Notice that $\mathcal{U}_{2}(\lambda) F_{\lambda}\left(h_{1}, h_{2}\right)=\mathbf{U}_{2}(\lambda)\left(h_{1}, h_{2}\right)$. We then define an operator $\mathcal{D}(\lambda)$ by

$$
\mathcal{D}(\lambda) F=\mathcal{U}_{2}(\lambda)\left(\mathbf{I}+F_{\lambda}\left(\mathcal{V}_{21}(\lambda), \mathcal{V}_{22}(\lambda)\right)\right)^{-1}
$$

for $F=\left(F_{1}, F_{2}, F_{3}\right) \in \mathcal{Y}_{q}(\Omega)$. Since

$$
\begin{aligned}
\left(\mathbf{I}+F_{\lambda}\left(\mathcal{V}_{21}(\lambda), \mathcal{V}_{22}(\lambda)\right)\right)^{-1} F_{\lambda} & =\sum_{j=0}^{\infty}\left(-F_{\lambda}\left(\mathcal{V}_{21}(\lambda), \mathcal{V}_{22}(\lambda)\right)\right)^{j} F_{\lambda}=F_{\lambda} \sum_{j=0}^{\infty}\left(-\left(\mathcal{V}_{21}(\lambda), \mathcal{V}_{22}(\lambda)\right) F_{\lambda}\right)^{j} \\
& =F_{\lambda}\left(\mathbf{I}+\left(\mathcal{V}_{21}(\lambda), \mathcal{V}_{22}(\lambda)\right) F_{\lambda}\right)^{-1}
\end{aligned}
$$

we have

$$
\begin{aligned}
\vartheta & =\mathbf{U}_{2}(\lambda)\left(\mathbf{I}+\left(\mathcal{V}_{21}(\lambda), \mathcal{V}_{22}(\lambda)\right) F_{\lambda}\right)^{-1}\left(h_{1}, h_{2}\right)=\mathcal{U}_{2}(\lambda) F_{\lambda}\left(\mathbf{I}+\left(\mathcal{V}_{21}(\lambda), \mathcal{V}_{22}(\lambda)\right) F_{\lambda}\right)^{-1}\left(h_{1}, h_{2}\right) \\
& =\mathcal{U}_{2}(\lambda)\left(\mathbf{I}+F_{\lambda}\left(\mathcal{V}_{21}(\lambda), \mathcal{V}_{22}(\lambda)\right)\right)^{-1} F_{\lambda}\left(h_{1}, h_{2}\right)=\mathcal{D}(\lambda) F_{\lambda}\left(h_{1}, h_{2}\right)=\mathcal{D}(\lambda)\left(h_{1}, \lambda^{1 / 2} h_{2}, h_{2}\right) .
\end{aligned}
$$

By (7.42) and (7.45), we have

$$
\mathcal{R}_{\mathcal{L}\left(\mathcal{Y}_{q}(\Omega), H_{q}^{2-k}(\Omega)\right)}\left(\left\{\left(\tau \partial_{\tau}\right)^{\ell}\left(\lambda^{k / 2} \mathcal{D}(\lambda)\right) \mid \lambda \in \Sigma_{\epsilon, \tilde{\lambda}_{0}}\right\}\right) \leq 4 C_{N, q} r_{b}
$$

This completes the proof of the assertion (2) of Theorem 15

\subsection{Proof of Theorem 13}

Let $\mathcal{C}(\lambda)$ and $\mathcal{D}(\lambda)$ be the operators given in Theorem 15, Let $\vartheta_{0}=\mathcal{D}(\lambda)\left(0, \lambda^{1 / 2} h_{2}, h_{2}\right)$, and then the third equation of Eq. (7.1) and the boundary condition for $\vartheta$ are reduced to the equations:

$$
\gamma_{3}(x) \lambda \varphi+\gamma_{2}(x) \operatorname{div} \mathbf{v}-\operatorname{div}\left(\gamma_{4}(x) \nabla \varphi\right)=f_{3} \quad \text { in } \Omega,\left.\quad(\nabla \varphi) \cdot \mathbf{n}\right|_{\Gamma}=0 .
$$

Thus, in view of (7.2) and (7.46), instead of (7.1) we consider the equations:

$$
\left\{\begin{array}{rlrl}
\rho_{0}(x) \lambda \mathbf{v}-\mu \Delta \mathbf{v}-\nu \nabla \operatorname{div} \mathbf{v}-\gamma_{1}(x) \lambda^{-1} \nabla\left(\rho_{0}(x) \operatorname{div} \mathbf{v}\right)+\gamma_{2}(x) \nabla \varphi & =\mathbf{f} & & \text { in } \Omega, \\
\gamma_{3}(x) \lambda \varphi+\gamma_{2}(x) \operatorname{div} \mathbf{v}-\operatorname{div}\left(\gamma_{4}(x) \nabla \varphi\right)=g & & \text { in } \Omega, \\
\mathbf{v}=0, \quad(\nabla \varphi) \cdot \mathbf{n}=0 & & \text { on } \Gamma .
\end{array}\right.
$$

In the following, we write $\mathcal{D}(\lambda)(g, 0,0)$ simply by $\mathcal{D}(\lambda) g$. Let

$$
\mathbf{v}=\mathcal{C}(\lambda) \mathbf{f}, \quad \varphi=\mathcal{D}(\lambda) g
$$

in (7.47), and then we have

$$
\left\{\begin{aligned}
\rho_{0}(x) \lambda \mathbf{v}-\mu \Delta \mathbf{v}-\nu \nabla \operatorname{div} \mathbf{v}-\gamma_{1}(x) \lambda^{-1} \nabla\left(\rho_{0}(x) \operatorname{div} \mathbf{v}\right)+\gamma_{2}(x) \nabla \varphi & =\mathbf{f}+\mathcal{E}_{1}(\lambda)(\mathbf{f}, g) & & \text { in } \Omega, \\
\gamma_{3}(x) \lambda \varphi+\gamma_{2}(x) \operatorname{div} \mathbf{v}-\operatorname{div}\left(\gamma_{4}(x) \nabla \varphi\right) & =g+\mathcal{E}_{2}(\lambda)(\mathbf{f}, g) & & \text { in } \Omega, \\
\mathbf{v}=0, \quad(\nabla \varphi) \cdot \mathbf{n} & =0 & & \text { on } \Gamma,
\end{aligned}\right.
$$


where we have set

$$
\mathcal{E}_{1}(\lambda)(\mathbf{f}, g)=\gamma_{3}(x) \nabla \mathcal{D}(\lambda) g, \quad \mathcal{E}_{2}(\lambda)(\mathbf{f}, g)=\gamma_{2}(x) \operatorname{div} \mathcal{C}(\lambda) \mathbf{f} .
$$

Let $\mathcal{E}(\lambda)(\mathbf{f}, g)=\left(\mathcal{E}_{1}(\lambda)(\mathbf{f}, g), \mathcal{E}_{2}(\lambda)(\mathbf{f}, g)\right)$, and then by Theorem 15 we have

$$
\mathcal{R}_{\mathcal{L}\left(L_{q}(\Omega)^{N+1}\right)}\left(\left\{\left(\tau \partial_{\tau}\right)^{\ell} \mathcal{E}(\lambda) \mid \lambda \in \Sigma_{\epsilon, \lambda_{1}}\right\}\right) \leq r_{b} \lambda_{1}^{-1 / 2}
$$

for any $\lambda_{1} \geq \lambda_{0}$. Thus, choosing $\lambda_{1}>0$ so large that $r_{b} \lambda_{1}^{-1 / 2} \leq 1 / 2$, by (7.49) and Lemma 19] we see that $(\mathbf{I}+\mathcal{E}(\lambda))^{-1}=\sum_{j=0}^{\infty}(-\mathcal{E}(\lambda))^{j}$ exists and satisfies the estimate:

$$
\mathcal{R}_{\mathcal{L}\left(L_{q}(\Omega)^{N L+1}\right)}\left(\left\{\left(\tau \partial_{\tau}\right)^{\ell}(\mathbf{I}+\mathcal{E}(\lambda))^{-1} \mid \lambda \in \Sigma_{\epsilon, \lambda_{1}}\right\}\right) \leq 4 .
$$

Let $\tilde{\mathcal{B}}_{1}(\lambda)=\mathcal{C}(\lambda)(\mathbf{I}+\mathcal{E}(\lambda))^{-1}$ and $\tilde{\mathcal{B}}_{2}(\lambda)=\mathcal{D}(\lambda)(\mathbf{I}+\mathcal{E}(\lambda))^{-1}$, and then by Theorem 15, (7.50), and Lemma 19, we see that for any $\lambda \in \Sigma_{\epsilon, \lambda_{1}}$ and $(\mathbf{f}, g) \in L_{q}(\Omega)^{N+1}, \mathbf{v}=\tilde{\mathcal{B}}_{1}(\lambda)(\mathbf{f}, g)$ and $\varphi=\tilde{\mathcal{B}}_{2}(\lambda)(\mathbf{f}, g)$ are solutions of (17.48) and

$$
\mathcal{R}_{\mathcal{L}\left(L_{q}(\Omega)^{N+1}, H_{q}^{2-k}(\Omega)^{N+1}\right)}\left(\left\{\left(\tau \partial_{\tau}\right)^{\ell}\left(\lambda^{k / 2}\left(\tilde{\mathcal{B}}_{1}(\lambda), \tilde{\mathcal{B}}_{2}(\lambda)\right)\right) \mid \lambda \in \Sigma_{\epsilon, \lambda_{1}}\right\}\right) \leq 4 r_{b} .
$$

Finally, setting

$$
\begin{aligned}
& \mathbf{v}=\tilde{\mathcal{B}}_{1}(\lambda)\left(\mathbf{f}_{2}-\lambda^{-1} \gamma_{1}(x) \nabla f_{1}, f_{3}\right), \\
& \zeta=\lambda^{-1}\left(f_{1}-\rho_{0}(x) \operatorname{div} \mathbf{v}\right) \\
& \vartheta=\tilde{\mathcal{B}}_{2}(\lambda)\left(\mathbf{f}_{2}-\lambda^{-1} \gamma_{1}(x) \nabla f_{1}, f_{3}\right)+\mathcal{D}(\lambda)\left(0, \lambda^{1 / 2} f_{4}, f_{4}\right)
\end{aligned}
$$

we see that $\zeta, \mathbf{v}$, and $\vartheta$ are solutions of Eq. (7.1). The uniqueness of solutions follows from the existence of solutions for the dual problem. For $F=\left(F_{1}, F_{2}, F_{3}, F_{4}, F_{5}\right) \in \mathcal{X}_{q}(\Omega)$, we set

$$
\begin{aligned}
\mathcal{B}_{1}(\lambda) F & =\tilde{\mathcal{B}}_{1}(\lambda)\left(F_{2}, F_{3}\right)-\lambda^{-1} \tilde{\mathcal{B}}_{1}(\lambda)\left(\gamma_{1}(x) \nabla F_{1}, 0\right), \\
\mathcal{A}(\lambda) F & =\lambda^{-1} F_{1}-\lambda^{-1} \rho_{0}(x) \operatorname{div} \mathcal{B}_{1}(\lambda) F, \\
\mathcal{B}_{2}(\lambda) F & =\tilde{\mathcal{B}}_{2}(\lambda)\left(F_{2}, F_{3}\right)-\lambda^{-1} \tilde{\mathcal{B}}_{2}(\lambda)\left(\gamma_{1}(x) \nabla F_{1}, 0\right)+\mathcal{D}(\lambda)\left(0, F_{4}, F_{5}\right),
\end{aligned}
$$

and then, we have $\zeta=\mathcal{A}(\lambda) \mathbf{F}_{\lambda}, \mathbf{v}=\mathcal{B}_{1}(\lambda) \mathbf{F}_{\lambda}$, and $\vartheta=\mathcal{B}_{2}(\lambda) \mathbf{F}_{\lambda}$, where $\mathbf{F}_{\lambda}=\left(f_{1}, \mathbf{f}_{2}, f_{3}, \lambda^{1 / 2} f_{4}, f_{4}\right)$. Moreover, by Lemma 20, (5.2) and (7.51) we have

$$
\begin{aligned}
\mathcal{R}_{\mathcal{L}\left(\mathcal{X}_{q}(\Omega), H_{q}^{2-k}(\Omega)^{N}\right)}\left(\left\{\left(\tau \partial_{\tau}\right)^{\ell}\left(\lambda^{k / 2} \mathcal{B}_{1}(\lambda)\right) \mid \lambda \in \Sigma_{\epsilon, \lambda_{1}}\right\}\right) & \leq\left(4+\lambda_{1}^{-1} \alpha_{4}\right) r_{b}, \\
\mathcal{R}_{\mathcal{L}\left(\mathcal{X}_{q}(\Omega), H_{q}^{2-k}(\Omega)\right)}\left(\left\{\left(\tau \partial_{\tau}\right)^{\ell}\left(\lambda^{k / 2} \mathcal{B}_{2}(\lambda)\right) \mid \lambda \in \Sigma_{\epsilon, \lambda_{1}}\right\}\right) & \leq\left(4+\lambda_{1}^{-1} \alpha_{4}\right) r_{b}, \\
\mathcal{R}_{\mathcal{L}\left(\mathcal{X}_{q}(\Omega), H_{q}^{1}(\Omega)\right)}\left(\left\{\left(\tau \partial_{\tau}\right)^{\ell}(\lambda \mathcal{A}(\lambda)) \mid \lambda \in \Sigma_{\epsilon, \lambda_{1}}\right\}\right) & \leq 1+\left(4+\lambda_{1}^{-1} \alpha_{4}\right) r_{b} .
\end{aligned}
$$

This completes the proof of Theorem 13 .

\subsection{Proof of Theorem 3}

We first prove the generation of a $C_{0}$ analytic semigroup associated with Eq. (5.1). Let

$$
\begin{aligned}
& \mathcal{D}_{q}(\Omega)=\left\{(\zeta, \mathbf{v}, \vartheta) \in D_{q}(\Omega)|\mathbf{v}|_{\Gamma}=0,\left.\quad(\nabla \vartheta) \cdot \mathbf{n}\right|_{\Gamma}=0\right\}, \\
& -\rho_{0}(x) \operatorname{div} \mathbf{v} \\
& A(\zeta, \mathbf{v}, \vartheta)=\left(\begin{array}{c}
\rho_{0}(x)^{-1}\left(\mu \Delta \mathbf{v}+\nu \nabla \operatorname{div} \mathbf{v}-\gamma_{1}(x) \nabla \zeta-\gamma_{2}(x) \nabla \vartheta\right) \\
\gamma_{3}(x)^{-1}\left(-\gamma_{2}(x) \operatorname{div} \mathbf{v}+\operatorname{div}\left(\gamma_{4}(x) \nabla \vartheta\right)\right)
\end{array}\right), \\
& \mathcal{A}_{q}(\zeta, \mathbf{v}, \vartheta)=A(\zeta, \mathbf{v}, \vartheta) \text { for }(\zeta, \mathbf{v}, \vartheta) \in \mathcal{D}_{q}(\Omega) .
\end{aligned}
$$

And then, Eq. (5.1) with $f_{1}=\mathbf{f}_{2}=f_{3}=g=0$ is formally written as

$$
\partial_{t} U-\mathcal{A}_{q} U=0 \text { for } t>0,\left.\quad U\right|_{t=0}=U_{0},
$$


where $U_{0}=\left(\zeta_{0}, \mathbf{v}_{0}, \vartheta_{0}\right) \in \mathcal{H}_{q}(\Omega)$ and $U$ with

$$
U \in C^{0}\left[0, \infty, \mathcal{H}_{q}(\Omega)\right) \cap C^{0}\left((0, \infty), \mathcal{D}_{q}(\Omega) \cap C^{1}\left((0, \infty), \mathcal{H}_{q}(\Omega)\right) .\right.
$$

The resolvent equation corresponding to (7.53) is

$$
\lambda V-\mathcal{A}_{q} V=F \quad \text { in } \Omega,
$$

where $F=\left(f_{1}, \mathbf{f}_{2}, f_{3}\right) \in \mathcal{H}_{q}(\Omega)$ and $V \in \mathcal{D}_{q}(\Omega)$. By Theorem [13, we see that the resolvent set $\rho\left(\mathcal{A}_{q}\right)$ of $\mathcal{A}_{q}$ contains $\Sigma_{\epsilon, \lambda_{0}}$ and for any $F \in \mathcal{H}_{q}(\Omega)$ and $\lambda \in \Sigma_{\epsilon, \lambda_{0}}, V=\left(\lambda \mathbf{I}-\mathcal{A}_{q}\right)^{-1} F \in \mathcal{D}_{q}(\Omega)$ satisfies the estimate:

$$
|\lambda|\|V\|_{\mathcal{H}_{q}(\Omega)}+\|V\|_{\mathcal{D}_{q}(\Omega)} \leq r_{b}\|F\|_{\mathcal{H}_{q}(\Omega)}
$$

where

$$
\|F\|_{\mathcal{H}_{q}(\Omega)}=\left\|f_{1}\right\|_{H_{q}^{1}(\Omega)}+\left\|\left(\mathbf{f}_{2}, f_{3}\right)\right\|_{L_{q}(\Omega)}, \quad\|V\|_{\mathcal{D}_{q}(\Omega)}=\|\zeta\|_{H_{q}^{1}(\Omega)}+\|(\mathbf{v}, \vartheta)\|_{H_{q}^{2}(\Omega)}
$$

for $F=\left(f_{1}, \mathbf{f}_{2}, f_{3}\right) \in \mathcal{H}_{q}(\Omega)$ and $V=(\zeta, \mathbf{v}, \vartheta) \in \mathcal{D}_{q}(\Omega)$. Since $0<\epsilon<\pi / 2$, the operator $\mathcal{A}_{q}$ generates a $C_{0}$ analytic semigroup $\{T(t)\}_{t \geq 0}$ on $\mathcal{H}_{q}(\Omega)$ possessing the estimate

$$
\|T(t) F\|_{\mathcal{H}_{q}(\Omega)} \leq C e^{\gamma t}\|F\|_{\mathcal{H}_{q}(\Omega)} \quad(t>0)
$$

for some constants $C$ and $\gamma$. Let

We now consider the maximal $L_{p}-L_{q}$ regularity for Eq. (5.1) in the case that $f_{1}=\mathbf{f}_{2}=f_{3}=g=0$.

$$
\mathcal{E}_{p, q}(\Omega)=\left(\mathcal{H}_{q}(\Omega), \mathcal{D}_{q}(\Omega)\right)_{1-1 / p, p} .
$$

Notice that $\mathcal{E}_{p, q}(\Omega) \subset D_{p, q}(\Omega)$, and that for $(\zeta, \mathbf{v}, \vartheta) \in \mathcal{E}_{p, q}(\Omega)$ we have

$$
\left.\mathbf{v}\right|_{\Gamma}=0 \quad \text { for } 2 / p+1 / q<2,\left.\quad(\nabla \vartheta) \cdot \mathbf{n}\right|_{\Gamma}=0 \quad \text { for } 2 / p+1 / q<1 .
$$

By real interpolation theory, we have

Theorem 25. Let $1<p, q<\infty$. Assume that $\Omega$ is a uniformly $C^{2}$ domain. Then, for $\left(\zeta_{0}, \mathbf{v}_{0}, \vartheta_{0}\right) \in$ $\mathcal{E}_{p, q}(\Omega),(\zeta, \mathbf{v}, \vartheta)=T(t)\left(\zeta_{0}, \mathbf{v}_{0}, \vartheta_{0}\right)$ satisfies Eq. (5.1) with $f_{1}=\mathbf{f}_{2}=f_{3}=g=0$ and possesses the estimate:

$$
\left\|e^{-\gamma t}(\zeta, \mathbf{v}, \vartheta)\right\|_{H_{p}^{1}\left((0, \infty), \mathcal{H}_{q}(\Omega)\right)}+\left\|e^{-\gamma t}(\mathbf{v}, \vartheta)\right\|_{L_{p}\left((0, \infty), H_{q}^{2}(\Omega)\right)} \leq C\left\|\left(\zeta_{0}, \mathbf{v}_{0}, \vartheta_{0}\right)\right\|_{D_{p, q}(\Omega)} .
$$

Remark 26. Theorem 25 can be shown employing the same argument as that in the proof of Theorem 3.9 in Shibata-Shimizu [41], so we may omit the proof.

We next consider Eq. (5.1) in the case that $\left(\zeta_{0}, \mathbf{v}_{0}, \vartheta_{0}\right)=0$. Notice that $g$ is defined on $\mathbb{R}$ with respect to $t$. We extend $f_{1}, \mathbf{f}_{2}$, and $f_{3}$ to $\mathbb{R}$ as follows:

$$
f_{10}(\cdot, t)=\left\{\begin{array}{ll}
f_{1}(\cdot, t) & t \in(0, T), \\
0 & t \notin(0, T),
\end{array} \mathbf{f}_{20}(\cdot, t)=\left\{\begin{array}{ll}
\mathbf{f}_{2}(\cdot, t) & t \in(0, T), \\
0 & t \notin(0, T),
\end{array} \quad f_{30}(\cdot, t)= \begin{cases}f_{3}(\cdot, t) & t \in(0, T), \\
0 & t \notin(0, T) .\end{cases}\right.\right.
$$

We then consider the following equations:

$$
\partial_{t} V_{1}-A V_{1}=\left(f_{10}, \mathbf{f}_{20}, f_{30}\right) \quad \text { in } \Omega \times \mathbb{R}, \quad \mathbf{v}_{1}=0, \quad\left(\nabla \vartheta_{1}\right) \cdot \mathbf{n}=g \quad \text { on } \Gamma \times \mathbb{R},
$$

where $V_{1}=\left(\zeta_{1}, \mathbf{v}_{1}, \vartheta_{1}\right)$. We use the Laplace transform $\mathcal{L}$ with respect to $t$ and its inversion formula $\mathcal{L}^{-1}$, which are defined by

$$
\begin{aligned}
\mathcal{L}[f](\cdot, \lambda) & =\int_{-\infty}^{\infty} e^{-\lambda t} f(\cdot, t) d t=\mathcal{F}\left[e^{-\gamma t} f\right](\tau) \quad(\lambda=\gamma+i \tau \in \mathbb{C}), \\
\mathcal{L}^{-1}[g](\cdot, t) & =\frac{1}{2 \pi} \int_{-\infty}^{\infty} e^{\lambda t} g(\cdot, \tau) d \tau=e^{\gamma t} \mathcal{F}^{-1}[g](\cdot, t) .
\end{aligned}
$$


where $\mathcal{F}$ and $\mathcal{F}^{-1}$ denote the Fourier transform with respect to $t$ and its inverse. Applying the Laplace transform to Eq. (7.58), we have

$$
\lambda \hat{V}_{1}-A \hat{V}_{1}=\left(\mathcal{L}\left[f_{10}\right], \mathcal{L}\left[\mathbf{f}_{20}\right], \mathcal{L}\left[f_{30}\right]\right) \quad \text { in } \Omega, \quad \hat{\mathbf{v}}_{1}=0, \quad\left(\nabla \hat{\vartheta}_{1}\right) \cdot \mathbf{n}=\mathcal{L}[g] \quad \text { on } \Gamma .
$$

Let $\mathcal{S}(\lambda)=\left(\mathcal{A}(\lambda), \mathcal{B}_{1}(\lambda), \mathcal{B}_{2}(\lambda)\right)$ be $\mathcal{R}$ bounded solution operators given in Theorem 13, We then have $\hat{V}_{1}(\lambda)=\mathcal{S}(\lambda) \mathbf{F}_{\lambda}$, where we have set

$$
\mathbf{F}_{\lambda}=\left(\mathcal{L}\left[f_{10}\right](\lambda), \mathcal{L}\left[\mathbf{f}_{20}\right](\lambda), \mathcal{L}\left[f_{30}\right](\lambda), \lambda^{1 / 2} \mathcal{L}[g](\lambda), \mathcal{L}[g](\lambda)\right) .
$$

We now introduce an operator $\Lambda_{\gamma}^{1 / 2}$ by

$$
\Lambda_{\gamma}^{1 / 2} g=\mathcal{L}^{-1}\left[\lambda^{1 / 2} \mathcal{L}[g](\lambda)\right] .
$$

Since

$$
\left|\left(\tau \partial_{\tau}\right)^{\ell}\left(\lambda^{1 / 2} /\left(1+\tau^{2}\right)^{1 / 4}\right)\right| \leq C_{\gamma}
$$

for any $\lambda=\gamma+i \tau \in \mathbb{C}$ with some constant $C_{\gamma}$ depending solely on $\gamma \in \mathbb{R}$, by Bourgain theorem (cf. Lemma 20), we have

$$
\left\|e^{-\gamma t} \Lambda_{\gamma}^{1 / 2} g\right\|_{L_{p}\left(\mathbb{R}, L_{q}(\Omega)\right)} \leq C_{\gamma}\left\|e^{-\gamma t} g\right\|_{H_{p}^{1 / 2}\left(\mathbb{R}, L_{q}(\Omega)\right)}
$$

for any $\gamma>0$. Since $\lambda^{1 / 2} \mathcal{L}[g](\lambda)=\mathcal{L}\left[\Lambda_{\gamma}^{1 / 2} g\right](\lambda)$, using Theorem [13 and Weis' operator valued Fourier multiplier theorem [47, we have

$$
\begin{aligned}
& \left\|e^{-\gamma t}\left(\zeta_{1}, \mathbf{v}_{1}, \vartheta_{1}\right)\right\|_{H_{p}^{1}\left(\mathbb{R}, \mathcal{H}_{q}(\Omega)\right)}+\left\|e^{-\gamma t}\left(\mathbf{v}_{1} \vartheta_{1}\right)\right\|_{L_{p}\left(\mathbb{R}, H_{q}^{2}(\Omega)\right)} \\
& \quad \leq r_{b}\left(\left\|e^{-\gamma t}\left(f_{10}, \mathbf{f}_{20}, f_{30}\right)\right\|_{L_{p}\left(\mathbb{R}, \mathcal{H}_{q}(\Omega)\right)}+\left\|e^{-\gamma t} \Lambda_{\gamma}^{1 / 2} g\right\|_{L_{p}\left(\mathbb{R}, L_{q}(\Omega)\right)}+\left\|e^{-\gamma t} g\right\|_{L_{p}\left(\mathbb{R}, H_{q}^{1}(\Omega)\right)}\right),
\end{aligned}
$$

which, combined with (7.60), leads to

$$
\begin{aligned}
& \left\|\left(\zeta_{1}, \mathbf{v}_{1}, \vartheta_{1}\right)\right\|_{H_{p}^{1}\left((0, T), \mathcal{H}_{q}(\Omega)\right)}+\left\|\left(\mathbf{v}_{1}, \vartheta_{1}\right)\right\|_{L_{p}\left((0, T), H_{q}^{2}(\Omega)\right)} \\
& \quad \leq r_{b} e^{\gamma T}\left(\left\|\left(f_{1}, \mathbf{f}_{2}, f_{3}\right)\right\|_{L_{p}\left((0, T), \mathcal{H}_{q}(\Omega)\right)}+C_{\gamma}\left\|e^{-\gamma t} g\right\|_{H_{p}^{1 / 2}\left(\mathbb{R}, L_{q}(\Omega)\right)}+\left\|e^{-\gamma t} g\right\|_{L_{p}\left(\mathbb{R}, H_{q}^{1}(\Omega)\right)}\right) .
\end{aligned}
$$

Finally, let $V_{2}$ be a solution of the system:

$$
\left\{\begin{aligned}
\partial_{t} V_{2}-A V_{2} & =0 & & \text { in } \Omega \times(0, \infty), \\
\mathbf{v}_{2}=0, \quad\left(\nabla \vartheta_{2}\right) \cdot \mathbf{n} & =0 & & \text { on } \Gamma \times(0, \infty), \\
V_{2} & =V_{0}-\left.V_{1}\right|_{t=0} & & \text { in } \Omega .
\end{aligned}\right.
$$

By the compatibility condition, $V_{0}-\left.V_{1}\right|_{t=0} \in \mathcal{D}_{p, q}(\Omega)$ provided that $2 / p+1 / q \neq 1$ and $2 / p+1 / q \neq 1$. Thus, by Theorem $\left[25\right.$ we see that $V_{2}=\left(\zeta_{2}, \mathbf{v}_{2}, \vartheta_{2}\right)$ exists and satisfies the following estimate:

$$
\begin{aligned}
& \left\|e^{-\gamma t}\left(\zeta_{2}, \mathbf{v}_{2}, \vartheta_{2}\right)\right\|_{H_{p}^{1}\left((0, \infty), \mathcal{H}_{q}(\Omega)\right)}+\left\|e^{-\gamma t}\left(\mathbf{v}_{2}, \vartheta_{2}\right)\right\|_{L_{p}\left((0, \infty), H_{q}^{2}(\Omega)\right)} \\
& \quad \leq C\left(\left\|\left(\zeta_{0}-\left.\zeta_{1}\right|_{t=0}, \mathbf{v}_{0}-\left.\mathbf{v}_{1}\right|_{t=0}, \vartheta_{0}-\left.\vartheta_{1}\right|_{t=0}\right)\right\|_{D_{p, q}(\Omega)}\right) .
\end{aligned}
$$

By real interpolation theorem, we have

$$
\left\|\left(\left.\mathbf{v}_{1}\right|_{t=0},\left.\vartheta_{1}\right|_{t=0}\right)\right\|_{B_{q, p}^{2(1-1 / p)}(\Omega)} \leq C\left(\left\|e^{-\gamma t} \partial_{t}\left(\mathbf{v}_{1}, \vartheta_{1}\right)\right\|_{L_{p}\left((0, \infty), L_{q}(\Omega)\right)}+\left\|e^{-\gamma t}\left(\mathbf{v}_{1}, \vartheta_{1}\right)\right\|_{L_{p}\left((0, \infty), H_{q}^{2}(\Omega)\right)}\right)
$$

because $\left.e^{-\gamma t}\left(\mathbf{v}_{1}, \vartheta_{1}\right)\right|_{t=0}=\left(\left.\mathbf{v}_{1}\right|_{t=0},\left.\vartheta_{1}\right|_{t=0}\right)$. Putting $\zeta=\zeta_{1}+\zeta_{2}, \mathbf{v}=\mathbf{v}_{1}+\mathbf{v}_{2}$ and $\vartheta=\vartheta_{1}+\vartheta_{2}$, we see that $\zeta, \mathbf{v}$ and $\vartheta$ are required solutios of Eq. (5.1). The uniqueness follows from the existence of solutions for the dual problem (cf. Shibata-Shimizu [41, Proof of Theorem 4.3]). This completes the proof of Theorem 3 . 


\section{Decay Estimate - proof of Theorem 10}

To prove Theorem 10, we first prove the existence of a $C^{0}$ analytic semigroup associated with Eq. (6.4) that is exponentially stable. For this purpose, we consider the resolvent problem:

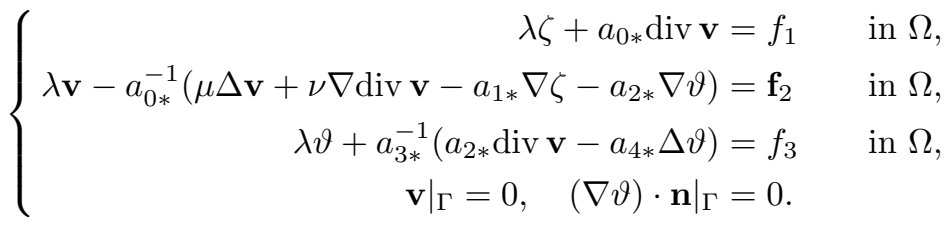

We shall prove

Theorem 27. Let $1<q<\infty$ and $0<\epsilon<\pi / 2$. Assume that $\Omega$ is a bounded domain in $\mathbb{R}^{N}(N \geq 2)$ whose boundary $\Gamma$ is a compact hypersurface of $C^{2}$ class. Assume that $a_{0 *}, a_{1 *}, \mu, \nu, a_{3 *}$, and $a_{4 *}$ are positive constans and that $a_{2 *}$ is a non-zero constant. Let

$$
\hat{\mathcal{H}}_{q}(\Omega)=\left\{\left(f_{1}, \mathbf{f}_{2}, f_{3}\right) \in \mathcal{H}_{q}(\Omega) \mid \int_{\Omega} f_{1} d x=\int_{\Omega} f_{3} d x=0\right\} .
$$

Set $\mathbf{C}_{+}=\{\lambda \in \mathbb{C} \mid \operatorname{Re} \lambda \geq 0\}$. Then, for any $\lambda \in \mathbf{C}_{+}$and $\left(f_{1}, \mathbf{f}_{2}, f_{3}\right) \in \hat{H}_{q}(\Omega)$, problem (8.1) admits a unique solution $U=(\zeta, \mathbf{v}, \vartheta) \in D_{q}(\Omega) \cap \hat{\mathcal{H}}_{q}(\Omega)$ possessing the esitmate:

$$
(|\lambda|+1)\|(\zeta, \mathbf{v}, \vartheta)\|_{\mathcal{H}_{q}(\Omega)}+\|(\mathbf{v}, \vartheta)\|_{H_{q}^{2}(\Omega)} \leq C\left\|\left(f_{1}, \mathbf{f}_{2}, f_{3}\right)\right\|_{\mathcal{H}_{q}(\Omega)} .
$$

Proof. Employing the same argument as that in the proof of Theorem 13, we can prove the existence of $\mathcal{R}$-bounded solution operators corresponding to Eq. (8.1), and so there exists $\lambda_{0} \geq 1$ such that for any $\lambda \in \Sigma_{\epsilon, \lambda_{0}}$ and $\left(f_{1}, \mathbf{f}_{2}, f_{3}\right) \in \mathcal{H}_{q}(\Omega)$, problem (8.1) admits a unique solution $(\zeta, \mathbf{v}, \vartheta) \in D_{q}(\Omega)$ possessing the estimate (8.3). Moreover, if $f_{1}$ and $f_{3}$ satisfy zero average condition, then $\zeta$ and $\vartheta$ also satisfy this condition in the case that $\lambda \neq 0$, what can be easily observed intergating (8.1) 1 and $(8.1)_{3}$ and applying the boundary conditions. Thus, for $\lambda \in \Sigma_{\epsilon, \lambda_{0}}$ the solutions obtained above belong to $\hat{\mathcal{H}}_{q}(\Omega)$.

Let $\mathcal{B}_{\lambda_{0}}=\left\{\lambda \in \mathbb{C}|\operatorname{Re} \lambda \geq 0,| \lambda \mid \leq \lambda_{0}\right\}$. Our task now is to prove the unique existence theorem for $\lambda \in \mathcal{B}_{\lambda_{0}}$. We first consider the case where $\lambda \neq 0$. Inserting the formula $\zeta=\lambda^{-1}\left(f_{1}-a_{* 0} \operatorname{div} \mathbf{v}\right)$ into the second equation in Eq. (8.1), it becomes

$$
\lambda \mathbf{v}-a_{0 *}^{-1}\left\{\mu \Delta \mathbf{v}+\left(\nu+\lambda^{-1} a_{1 *} a_{0 *}\right) \nabla \operatorname{div} \mathbf{v}-a_{2 *} \nabla \vartheta\right\}=\mathbf{f}_{2}-a_{0 *}^{-1} a_{1 *} \lambda^{-1} \nabla f_{1} .
$$

Thus, we consider the following equations:

$$
\left\{\begin{array}{rlrl}
\lambda \mathbf{v}-a_{0 *}^{-1}\left\{\mu \Delta \mathbf{v}+\left(\nu+\lambda^{-1} a_{1 *} a_{0 *}\right) \nabla \operatorname{div} \mathbf{v}\right\}+a_{0 *}^{-1} a_{2 *} \nabla \vartheta & =\mathbf{f}_{2} & & \text { in } \Omega, \\
\lambda \vartheta+a_{2 *} a_{3 *}^{-1} \operatorname{div} \mathbf{v}-a_{3 *}^{-1} a_{4 *} \Delta \vartheta & =f_{3} & & \text { in } \Omega, \\
\left.\mathbf{v}\right|_{\Gamma}=0, & \left.(\nabla \vartheta) \cdot \mathbf{n}\right|_{\Gamma}=0 . &
\end{array}\right.
$$

To solve Eq. (8.4), we introduce a new resolvent parameter $\tau>0$ and we consider auxiliary problem:

$$
\tau(\mathbf{v}, \vartheta)-\mathcal{A}_{\lambda}(\mathbf{v}, \vartheta)=\left(\mathbf{g}_{1}, g_{2}\right) \quad \text { in } \Omega,
$$

where we have set

$$
\begin{aligned}
\mathcal{A}_{\lambda}(\mathbf{v}, \vartheta) & =\left(A_{1 \lambda} \mathbf{v}-a_{0 *}^{-1} a_{2 *} \nabla \vartheta, a_{3 *}^{-1} a_{4 *} \Delta \vartheta-a_{2 *} a_{3 *}^{-1} \operatorname{div} \mathbf{v}\right) \quad \text { for }(\mathbf{v}, \vartheta) \in \mathcal{D}_{q}^{1}(\Omega) \times \mathcal{D}_{q}^{2}(\Omega), \\
\mathcal{D}_{q}^{1}(\Omega) & =\left\{\mathbf{v} \in H_{q}^{2}(\Omega)^{N}|\mathbf{v}|_{\Gamma}=0\right\}, \quad \mathcal{D}_{q}^{2}(\Omega)=\left\{\vartheta \in H_{q}^{2}(\Omega)|(\nabla \vartheta) \cdot \mathbf{n}|_{\Gamma}=0\right\}, \\
A_{1 \lambda} \mathbf{v} & =a_{0 *}^{-1}\left(\mu \Delta \mathbf{v}+\left(\nu+\lambda^{-1} a_{1 *} a_{0 *}\right) \nabla \operatorname{div} \mathbf{v}\right) \quad \text { for } \mathbf{v} \in H_{q}^{2}(\Omega) .
\end{aligned}
$$

Let

$$
\begin{aligned}
& \mathcal{A}_{1 \lambda} \mathbf{v}=a_{0 *}^{-1}\left(\mu \Delta \mathbf{v}+\left(\nu+\lambda^{-1} a_{1 *} a_{0 *}\right) \nabla \operatorname{div} \mathbf{v}\right) \quad \text { for } \mathbf{v} \in \mathcal{D}_{q}^{1}(\Omega), \\
& \mathcal{A}_{2} \vartheta=a_{3 *}^{-1} a_{4 *} \vartheta \vartheta \quad \text { for } \vartheta \in \mathcal{D}_{q}^{2}(\Omega) \text {. }
\end{aligned}
$$


Shibata and Tanaka [42] proved that there exists a $\tau_{0}>0$ such that $\left(\tau \mathbf{I}-\mathcal{A}_{1 \lambda}\right)^{-1}$ exists as a bounded linear operator from $L_{q}(\Omega)^{N}$ into $\mathcal{D}_{q}^{1}(\Omega)$ for $\tau \geq \tau_{0}$ possessing the estimate:

$$
\tau\|\mathbf{w}\|_{L_{q}(\Omega)}+\tau^{1 / 2}\|\mathbf{w}\|_{H_{q}^{1}(\Omega)}+\|\mathbf{w}\|_{H_{q}^{2}(\Omega)} \leq C\left\|\mathbf{g}_{1}\right\|_{L_{q}(\Omega)}
$$

for any $\tau \geq \tau_{0}$ and $\mathbf{g}_{1} \in L_{q}(\Omega)^{N}$, where we have set $\mathbf{w}=\left(\tau \mathbf{I}-\mathcal{A}_{1 \lambda}\right)^{-1} \mathbf{g}_{1}$. And, by Theorem [15] we see that there exists a $\tau_{0}>0$ such that $\left(\tau \mathbf{I}-\mathcal{A}_{2}\right)^{-1}$ exists as a bounded linear operator from $L_{q}(\Omega)$ into $\mathcal{D}_{q}^{2}(\Omega)$ for $\tau \geq \tau_{0}$ possessing the estimate:

$$
\tau\|\varphi\|_{L_{q}(\Omega)}+\tau^{1 / 2}\|\varphi\|_{H_{q}^{1}(\Omega)}+\|\varphi\|_{H_{q}^{2}(\Omega)} \leq C\left\|g_{2}\right\|_{L_{q}(\Omega)}
$$

for any $\tau \geq \tau_{0}$ and $g_{2} \in L_{q}(\Omega)$, where we have set $\varphi=\left(\tau \mathbf{I}-A_{2}\right)^{-1} \mathbf{g}_{1}$. To solve (8.5), we set $(\mathbf{v}, \vartheta)=$ $\left(\left(\tau \mathbf{I}-\mathcal{A}_{1 \lambda}\right)^{-1} \mathbf{g}_{1},\left(\tau \mathbf{I}-\mathcal{A}_{2}\right)^{-1} g_{2}\right)$. We then have

$$
\tau(\mathbf{v}, \vartheta)-\mathcal{A}_{\lambda}(\mathbf{v}, \vartheta)=\left(\mathbf{g}_{1}, g_{2}\right)+\mathcal{R}_{\tau}\left(\mathbf{g}_{1}, g_{2}\right)
$$

where we have set

$$
\mathcal{R}_{\tau}\left(\mathbf{g}_{1}, g_{2}\right)=\left(a_{0 *}^{-1} a_{2 *} \nabla\left(\tau \mathbf{I}-\mathcal{A}_{2}\right)^{-1} g_{2}, a_{2 *} a_{3 *}^{-1} \operatorname{div}\left(\tau \mathbf{I}-\mathcal{A}_{1}\right)^{-1} \mathbf{g}_{1}\right) .
$$

By (8.6) and (8.7), we have

$$
\left\|\mathcal{R}_{\tau}\left(\mathbf{g}_{1}, g_{2}\right)\right\|_{L_{q}(\Omega)} \leq C \tau^{-1 / 2}\left\|\left(\mathbf{g}_{1}, g_{2}\right)\right\|_{L_{q}(\Omega)},
$$

and so for large $\tau>0,\left(\mathbf{I}-\mathcal{R}_{\tau}\right)^{-1}$ exists as an element in $\mathcal{L}\left(L_{q}(\Omega)^{N+1}\right)$ and $\left\|\left(\mathbf{I}+\mathcal{R}_{\tau}\right)^{-1}\right\|_{\mathcal{L}\left(L_{q}(\Omega)^{N+1}\right)} \leq 2$. Let $\left(\mathbf{I}+\mathcal{R}_{\tau}\right)^{-1}\left(\mathbf{g}_{1}, g_{2}\right)=\left(\mathbf{h}_{1 \tau}, h_{2 \tau}\right)$, and then $\mathbf{v}_{\tau}=\left(\tau \mathbf{I}-\mathcal{A}_{1}\right) \mathbf{h}_{\tau 1} \in \mathcal{D}_{q}^{1}(\Omega)$ and $\vartheta_{\tau}=\left(\tau-\mathcal{A}_{2}\right)^{-1} h_{2 \tau} \in$ $\mathcal{D}_{q}^{2}(\Omega)$ are unique solutions of Eq. (8.5) possessing the estimate:

$$
\left.\tau\left\|\left(\mathbf{v}_{\tau}, \vartheta_{\tau}\right)\right\|_{L_{q}(\Omega)}+\| \mathbf{v}_{\tau}, \vartheta_{\tau}\right)\left\|_{H_{q}^{2}(\Omega)} \leq C\right\|\left(\mathbf{g}_{1}, g_{2}\right) \|_{L_{q}(\Omega)}
$$

for any large $\tau>0$. Namely, the resolvent set $\rho\left(\mathcal{A}_{\lambda}\right)$ of $\mathcal{A}_{\lambda}$ contains $\left(\tau_{1}, \infty\right)$ for some $\tau_{1}>0$. We then write the resolvent operator by $\left(\tau \mathbf{I}-\mathcal{A}_{\lambda}\right)^{-1}$ as usual. If we set $\left(\mathbf{v}_{\tau}, \vartheta_{\tau}\right)=\left(\mathbf{I}-\mathcal{A}_{\lambda}\right)^{-1}\left(\mathbf{g}_{1}, g_{2}\right)$, then $\left(\mathbf{v}_{\tau}, \vartheta_{\tau}\right)$ satisfies the estimate (8.9). Using $\left(\tau \mathbf{I}-\mathcal{A}_{\tau}\right)^{-1}$, we write Eq. (8.4) as

$$
(\mathbf{v}, \vartheta)+(\lambda-\tau)\left(\tau \mathbf{I}-\mathcal{A}_{\lambda}\right)^{-1}(\mathbf{v}, \vartheta)=\left(\tau \mathbf{I}-\mathcal{A}_{\lambda}\right)^{-1}\left(\mathbf{g}_{1}, g_{2}\right) .
$$

Since $(\lambda-\tau)\left(\tau \mathbf{I}-\mathcal{A}_{\lambda}\right)^{-1}$ is a compact operator on $L_{q}(\Omega)^{N+1}$, in view of Riesz-Schauder theory, in particular Fredholm alternative principle, it is sufficient to prove that the kernel of $\mathbf{I}+(\lambda-\tau)\left(\tau \mathbf{I}-\mathcal{A}_{\lambda}\right)^{-1}$ is trivial in order to prove the existence of $\left(\mathbf{I}+(\lambda-\tau)\left(\tau \mathbf{I}-\mathcal{A}_{\lambda}\right)^{-1}\right)^{-1} \in \mathcal{L}\left(L_{q}(\Omega)^{N+1}\right)$. Thus, let $\left(\mathbf{g}_{1}, g_{2}\right)$ be an element in $L_{q}(\Omega)^{N+1}$ for which

$$
\left(\mathbf{I}+(\lambda-\tau)\left(\tau \mathbf{I}-\mathcal{A}_{\lambda}\right)^{-1}\right)\left(\mathbf{g}_{1}, g_{2}\right)=(0,0) .
$$

Since $\left(\mathbf{g}_{1}, g_{2}\right)=(\tau-\lambda)\left(\tau \mathbf{I}-\mathcal{A}_{\lambda}\right)^{-1}\left(\mathbf{g}_{1}, g_{2}\right) \in \mathcal{D}_{q}^{1}(\Omega) \times \mathcal{D}_{q}^{2}(\Omega)$, setting $(\mathbf{v}, \vartheta)=\left(\tau \mathbf{I}-\mathcal{A}_{\lambda}\right)\left(\mathbf{g}_{1}, g_{2}\right)$, we have

$$
(0,0)=\left(\tau-\mathcal{A}_{\lambda}\right)(\mathbf{v}, \vartheta)+(\lambda-\tau)(\mathbf{v}, \vartheta)=\left(\lambda \mathbf{I}-\mathcal{A}_{\lambda}\right)(\mathbf{v}, \vartheta),
$$

that is, $(\mathbf{v}, \vartheta) \in H_{q}^{2}(\Omega)^{N+1}$ satisfies the homogeneous equations:

$$
\left\{\begin{array}{rlrl}
a_{0 *} \lambda \mathbf{v}-\mu \Delta \mathbf{v}-\left(\nu+\lambda^{-1} a_{1 *} a_{0 *}\right) \nabla \operatorname{div} \mathbf{v}+a_{2 *} \nabla \vartheta & =0 & & \text { in } \Omega, \\
a_{3 *} \lambda \vartheta+a_{2 *} \operatorname{div} \mathbf{v}-a_{4 *} \Delta \vartheta & =0 & & \text { in } \Omega, \\
\left.\mathbf{v}\right|_{\Gamma}=0, & \left.(\nabla \vartheta) \cdot \mathbf{n}\right|_{\Gamma}=0 . &
\end{array}\right.
$$

To prove $(\mathbf{v}, \vartheta)=(0,0)$, we first consider the case where $2 \leq q<\infty$. Since $(\mathbf{v}, \vartheta) \in H_{q}^{2}(\Omega)^{N+1} \subset$ $H_{2}^{2}(\Omega)^{N+1}$, by (8.11) and the divergence theorem of Gauß we have

$$
\begin{aligned}
0= & a_{0 *} \lambda\|\mathbf{v}\|_{L_{2}(\Omega)}^{2}+\mu\|\nabla \mathbf{v}\|_{L_{2}(\Omega)}^{2}+\left(\nu+\lambda^{-1} a_{1 *} a_{0 *}\right)\|\operatorname{div} \mathbf{v}\|_{L_{2}(\Omega)}^{2} \\
& +a_{3 *} \lambda\|\vartheta\|_{L_{2}(\Omega)}^{2}+a_{4 *}\|\nabla \vartheta\|_{L_{2}(\Omega)}^{2}+a_{2 *}\left\{(\nabla \vartheta, \mathbf{v})_{\Omega}-(\mathbf{v}, \nabla \vartheta)_{\Omega}\right\}
\end{aligned}
$$


Taking the real part, we have

$$
\begin{aligned}
0= & a_{0 *} \operatorname{Re} \lambda\|\mathbf{v}\|_{L_{2}(\Omega)}^{2}+\mu\|\nabla \mathbf{v}\|_{L_{2}(\Omega)}^{2}+\left(\nu+a_{1 *} a_{0 *} \operatorname{Re} \lambda^{-1}\right)\|\operatorname{div} \mathbf{v}\|_{L_{2}(\Omega)}^{2} \\
& +a_{3 *} \operatorname{Re} \lambda\|\vartheta\|_{L_{2}(\Omega)}^{2}+a_{4 *}\|\nabla \vartheta\|_{L_{2}(\Omega)}^{2} .
\end{aligned}
$$

Since $\operatorname{Re} \lambda \geq 0$, we have $\nabla(\mathbf{v}, \vartheta)=(0,0)$ in $\Omega$, that is $\mathbf{v}$ and $\vartheta$ are constants. But, $\left.\mathbf{v}\right|_{\Gamma}=0$, and so $\mathbf{v}=0$. Thus, by the second equation and boundary condition $\left.(\nabla \vartheta) \cdot \mathbf{n}\right|_{\Gamma}=0$ in (8.11), we have

$$
0=a_{3 *} \lambda \int_{\Omega} \vartheta d x-a_{4 *} \int_{\Omega} \Delta \vartheta d x=a_{3 *} \lambda \int_{\Omega} \vartheta d x
$$

and so $\vartheta=0$. Thus, in the case that $2 \leq q<\infty$, we see that Eq. (8.4) admits a unique solution $(\mathbf{v}, \vartheta) \in \mathcal{D}_{q}^{1}(\Omega) \times \mathcal{D}_{q}^{2}(\Omega)$ possessing the estimate:

$$
\|(\mathbf{v}, \vartheta)\|_{H_{q}^{2}(\Omega)} \leq C_{\lambda}\left\|\left(\mathbf{f}_{2}, f_{3}\right)\right\|_{L_{q}(\Omega)}
$$

for some constant $C_{\lambda}$ depending on $\lambda$.

We next consider the case $1<q<2$. Let $q^{*}=q /(q-1) \in(2, \infty)$. For any $\left(\mathbf{g}_{1}, g_{2}\right) \in L_{q}(\Omega)^{N+1}$, let $(\mathbf{w}, \varphi) \in \mathcal{D}_{q *}^{1}(\Omega) \times \mathcal{D}_{q^{*}}^{2}(\Omega)$ be a solution of the equation:

$$
\left\{\begin{array}{rlrl}
\bar{\lambda} \mathbf{w}-a_{0 *}^{-1}\left\{\mu \Delta \mathbf{w}+\left(\nu+\bar{\lambda}^{-1} a_{1 *} a_{0 *}\right) \nabla \operatorname{div} \mathbf{w}\right\}-a_{0 *}^{-1} a_{2 *} \nabla \varphi=\mathbf{g}_{1} & & \text { in } \Omega, \\
\lambda \varphi-a_{2 *} a_{3 *}^{-1} \operatorname{div} \mathbf{w}-a_{3 *}^{-1} a_{4 *} \Delta \varphi & =g_{2} & & \text { in } \Omega, \\
\left.\mathbf{v}\right|_{\Gamma}=0, & \left.(\nabla \vartheta) \cdot \mathbf{n}\right|_{\Gamma}=0 . &
\end{array}\right.
$$

Replacing $\lambda$ and $a_{2 *}$ by $\bar{\lambda}$ and $-a_{2 *}$ in (8.4), we can prove the unique existence of solutions $(\mathbf{w}, \varphi) \in$ $\mathcal{D}_{q}^{1}(\Omega) \times \mathcal{D}_{q}^{2}(\Omega)$ of Eq. (8.13). By the divergence theorem of Gauß we have

$$
\begin{aligned}
0 & =\left(a_{0 *} \lambda \mathbf{v}-\mu \Delta \mathbf{v}-\left(\nu+\lambda^{-1} a_{1 *} a_{0 *}\right) \nabla \operatorname{div} \mathbf{v}+a_{2 *} \nabla \vartheta, \mathbf{w}\right)_{\Omega}+\left(a_{3 *} \lambda \vartheta+a_{2 *} \operatorname{div} \mathbf{v}-a_{4 *} \Delta \vartheta, \varphi\right)_{\Omega} \\
& =\left(\mathbf{v}, a_{0 *} \bar{\lambda} \mathbf{w}-\mu \mathbf{w}-\left(\nu+\bar{\lambda}^{-1} a_{1 *} a_{0 *}\right) \nabla \operatorname{div}\right)-\left(\vartheta, a_{2 *} \operatorname{div} \mathbf{w}\right)_{\Omega}+\left(\vartheta, a_{3 *} \bar{\lambda} \varphi-a_{4 *} \Delta \varphi\right)_{\Omega}-\left(\mathbf{v}, a_{2 *} \nabla \varphi\right) \\
& =a_{0 *}\left(\mathbf{v}, \mathbf{g}_{1}\right)_{\Omega}+a_{3 *}\left(\vartheta, g_{2}\right)_{\Omega} .
\end{aligned}
$$

Thus, the arbitrariness of $\left(\mathbf{g}_{1}, g_{2}\right) \in L_{q^{*}}(\Omega)^{N+1}$ yields $(\mathbf{v}, \vartheta)=(0,0)$, which leads to the unique existence of solutions $(\mathbf{v}, \vartheta) \in \mathcal{D}_{q}^{1}(\Omega) \times \mathcal{D}_{q}^{2}(\Omega)$ of Eq. (8.4) possessing the estimate (8.12). Thus, we have proved that for any $\lambda \in \mathcal{B}_{\lambda_{0}} \backslash\{0\}$ and $\left(f_{1}, \mathbf{f}_{2}, f_{3}\right) \in \mathcal{H}_{q}(\Omega)$ Eq. (8.1) admits a unique solution $(\zeta, \mathbf{v}, \vartheta) \in \mathcal{D}_{q}(\Omega)$ possessing the estimate:

$$
\| \zeta, \mathbf{v}, \vartheta)\left\|_{D_{q}(\Omega)} \leq C_{\lambda}\right\|\left(f_{1}, \mathbf{f}_{2}, f_{3}\right) \|_{\mathcal{H}_{q}(\Omega)} .
$$

We now consider the case that $\lambda=0$. Inserting the relation: $\operatorname{div} \mathbf{v}=a_{0 *}^{-1} f_{1}$, we rewrite (8.1) as

$$
\left\{\begin{aligned}
\operatorname{div} \mathbf{v} & =a_{0 *}^{-1} f_{1} & & \text { in } \Omega, \\
-\mu \Delta \mathbf{v}+a_{1 *} \nabla \zeta & =\mathbf{f}_{2}+\nu a_{0 *}^{-1} \nabla f_{1}-a_{2 *} \nabla \vartheta & & \text { in } \Omega, \\
-a_{4 *} \Delta \vartheta & =f_{3}-a_{0 *}^{-1} a_{2 *} f_{1} & & \text { in } \Omega, \\
\left.\mathbf{v}\right|_{\Gamma}=0,\left.\quad(\nabla \vartheta) \cdot \mathbf{n}\right|_{\Gamma} & =0 . & &
\end{aligned}\right.
$$

We first consider the Laplace equation:

$$
-a_{4 *} \Delta \vartheta=g_{2} \quad \text { in } \Omega,\left.\quad(\nabla \vartheta) \cdot \mathbf{n}\right|_{\Gamma}=0,
$$

and then, for any $g_{2} \in L_{q}(\Omega)$ with $\int_{\Omega} g_{2} d x=0$, problem (8.16) admits a unique solution $\vartheta \in H_{q}^{2}(\Omega)$ with $\int_{\Omega} \vartheta d x=0$ possessing the estimate: $\|\vartheta\|_{H_{q}^{2}(\Omega)} \leq C\left\|g_{2}\right\|_{L_{q}(\Omega)}$. Therefore the third equation of Eq. (8.15) admits a unique solution $\vartheta \in H_{q}^{2}(\Omega)$ satisfying the estimate: $\|\vartheta\|_{H_{q}^{2}(\Omega)} \leq C\left\|\left(f_{1}, f_{3}\right)\right\|_{L_{q}(\Omega)}$ and $\int_{\Omega} \vartheta d x=0$.

Finally, setting $g_{1}=a_{0 *}^{-1} f_{1}$ and $\mathbf{g}_{2}=\mathbf{f}_{2}-\nu a_{0 *}^{-1} \nabla f_{1}-a_{2 *} \nabla \vartheta$, we consider the Cattabriga problem:

$$
-\mu \Delta \mathbf{v}+a_{1 *} \nabla \zeta=\mathbf{g}_{2}, \quad \operatorname{div} \mathbf{v}=g_{1} \quad \text { in } \Omega,\left.\quad \mathbf{v}\right|_{\Gamma}=0 .
$$


By Farwig and Sohr [14, there exists a $\lambda_{0}>0$ for which the equation:

$$
\lambda_{0} \mathbf{v}-\mu \Delta \mathbf{v}+a_{1 *} \nabla \zeta=\mathbf{g}_{2}, \quad \operatorname{div} \mathbf{v}=g_{1} \quad \text { in } \Omega,\left.\quad \mathbf{v}\right|_{\Gamma}=0,
$$

admits a unique solution $(\zeta, \mathbf{v}) \in H_{q}^{1}(\Omega) \times H_{q}^{2}(\Omega)^{N}$ with $\int_{\Omega} \zeta d x=0$ for any $\left(g_{1}, \mathbf{g}_{2}\right) \in H_{q}^{1}(\Omega) \times L_{q}(\Omega)^{N}$ with $\int_{\Omega} g_{2} d x=0$. Thus, by the Fredholm alternative principle, the uniqueness of solutions of Eq. (8.17) yields the unique existence theorem, that is for any $\left(g_{1}, \mathbf{g}_{2}\right) \in H_{q}^{1}(\Omega) \times L_{q}(\Omega)^{N}$ with $\int_{\Omega} g_{2} d x=0$, problem (8.17) admits a unique solution $(\zeta, \mathbf{v}) \in H_{q}^{1}(\Omega) \times H_{q}^{2}(\Omega)^{N}$ with $\int_{\Omega} \zeta d x=0$ possessing the estimate:

$$
\|\zeta\|_{H_{q}^{1}(\Omega)}+\|\mathbf{v}\|_{H_{q}^{2}(\Omega)} \leq C\left(\left\|g_{1}\right\|_{H_{q}^{1}(\Omega)}+\left\|\mathbf{g}_{2}\right\|_{L_{q}(\Omega)}\right) .
$$

Therefore the problem of existence for (8.17) is reduced to showing uniqueness for the homogeneous problem which is an immediate consequence of the divergence theorem.

Summing up, we have proved that for any $\left(f_{1}, \mathbf{f}_{2}, f_{3}\right) \in \hat{\mathcal{H}}_{q}(\Omega)$, problem 8.15) admits a unique solution $(\zeta, \mathbf{v}, \vartheta) \in \mathcal{D}_{q}(\Omega) \cap \hat{\mathcal{H}}_{q}(\Omega)$ possessing the estimate:

$$
\|\zeta\|_{H_{q}^{1}(\Omega)}+\|(\mathbf{v}, \vartheta)\|_{H_{q}^{2}(\Omega)} \leq C\left(\left\|f_{1}\right\|_{H_{q}^{1}(\Omega)}+\left\|\left(\mathbf{f}_{2}, f_{3}\right)\right\|_{L_{q}(\Omega)}\right) .
$$

Since the resolvent operator is continuous and the set $\mathcal{B}_{\lambda_{0}}$ is compact, we can take the constants $C_{\lambda}$ in the estimate (8.14) independent of $\lambda \in \mathcal{B}_{\lambda_{0}}$. This completes the proof of Theorem 27.

We now give a

\section{Proof of Theorem 10, Let}

$$
\begin{aligned}
& P U=\left(\begin{array}{c}
-\rho_{0 *} \operatorname{div} \mathbf{v} \\
a_{0 *}^{-1}\left(\mu \Delta \mathbf{v}+\nu \nabla \operatorname{div} \mathbf{v}-a_{1 *} \nabla \zeta-a_{2} \nabla \vartheta\right) \\
-a_{3 *}^{-1}\left(a_{2 *} \operatorname{div} \mathbf{v}-a_{4 *} \Delta \vartheta\right)
\end{array}\right) \quad \text { for } U=(\zeta, \mathbf{v}, \vartheta) \in D_{q}(\Omega), \\
& \mathcal{P} U=P U \quad \text { for } U=(\zeta, \mathbf{v}, \vartheta) \in \mathcal{D}_{q}(\Omega) \cap \hat{\mathcal{H}}_{q}(\Omega) .
\end{aligned}
$$

Here, $\hat{\mathcal{H}}_{q}(\Omega)$ and $\mathcal{D}_{q}(\Omega)$ are the spaces given in (18.2) and (7.52), respectively. Let us consider the Cauchy problem:

$$
\partial_{t} U-\mathcal{P} U=0 \quad \text { for } t>0,\left.\quad U\right|_{t=0}=U_{0}=\left(\zeta_{0}, \mathbf{v}_{0}, \vartheta_{0}\right) \in \hat{\mathcal{H}}_{q}(\Omega) .
$$

The resolvent problem corresponding to (8.18) is Eq. (8.1). Thus, by Theorem 27 we see that $\mathcal{P}$ generates a $C_{0}$ analytic semigroup $\{\dot{T}(t)\}_{t \geq 0}$ that is exponentially stable on $\hat{\mathcal{H}}_{q}(\Omega)$, that is

$$
\left\|\dot{T}(t) U_{0}\right\|_{\mathcal{H}_{q}(\Omega)} \leq C e^{-\gamma_{1} t}\left\|U_{0}\right\|_{\mathcal{H}_{q}(\Omega)}
$$

for any $U_{0} \in \hat{\mathcal{H}}_{q}(\Omega)$ and $t>0$ with some positive constants $C$ and $\gamma_{1}$.

Let $\lambda_{1}>0$ be a sufficiently large number and let $\gamma>0$ be a small positive number determined later. We assume that

$$
0<\gamma<\gamma_{1}
$$

We consider the time-shifted equations:

$$
\left\{\begin{aligned}
\partial_{t} U_{1}+\lambda_{1} U_{1}-P U_{1} & =G & & \text { in } \Omega \times(0, T), \\
B U_{1} & =\left(0, g_{4}\right) & & \text { on } \Gamma \times(0, T), \\
\left.U_{1}\right|_{t=0} & =U_{0} & & \text { in } \Omega .
\end{aligned}\right.
$$

where $G=\left(g_{1}, \mathbf{g}_{2}, g_{3}\right)$ and $B U=(\mathbf{v},(\nabla \vartheta) \cdot \mathbf{n})$. Multiplying Eq. (8.21) by $e^{\gamma t}$, we have

$$
\left\{\begin{aligned}
\partial_{t}\left(e^{\gamma t} U_{1}\right)+\left(\lambda_{1}-\gamma\right) e^{\gamma t} U_{1}-P\left(e^{\gamma t} U_{1}\right) & =e^{\gamma t} G & & \text { in } \Omega \times(0, T), \\
B\left(e^{\gamma t} U_{1}\right) & =\left(0, e^{\gamma t} g_{4}\right) & & \text { on } \Gamma \times(0, T), \\
\left.e^{\gamma t} U_{1}\right|_{t=0} & =U_{0} & & \text { in } \Omega .
\end{aligned}\right.
$$


Let $G_{0}$ be the zero extension of $G$ to $\mathbb{R}$ with respect to $t$, that is $G_{0}(\cdot, t)=G(\cdot, t)$ for $t \in(0, T)$ and $G_{0}(\cdot, t)=0$ for $t \notin(0, T)$. To estimate $e^{\gamma t} U_{1}$, we consider the equations:

$$
\left\{\begin{aligned}
\partial_{t} U_{2}+\left(\lambda_{1}-\gamma\right) U_{2}-P U_{2} & =e^{\gamma t} G_{0} & & \text { in } \Omega \times \mathbb{R} \\
B U_{2} & =\left(0, e^{\gamma t} g_{4}\right) & & \text { on } \Gamma \times \mathbb{R} .
\end{aligned}\right.
$$

Applying the Fourier transform with respect to $t$ to Eq. (8.23), we have

$$
\left\{\begin{aligned}
\left(\lambda_{1}-\gamma+i \tau\right) \mathcal{F}\left[U_{2}\right](\cdot, \tau)- & P \mathcal{F}\left[U_{2}\right](\cdot, \tau)=\mathcal{F}\left[e^{\gamma t} G_{0}\right](\cdot, \tau) & & \text { in } \Omega, \\
B \mathcal{F}\left[U_{2}\right](\cdot, \tau) & =\left(0, \mathcal{F}\left[e^{\gamma t} g_{4}\right](\cdot, \tau)\right) & & \text { on } \Gamma .
\end{aligned}\right.
$$

Let $\mathcal{S}(\lambda)=\left(\mathcal{A}(\lambda), \mathcal{B}_{1}(\lambda), \mathcal{B}_{2}(\lambda)\right)$ be the $\mathcal{R}$-bounded solution operators given in Theorem 13 if we choose $\lambda_{1}>0$ so large that $\lambda_{1}-\gamma \geq \lambda_{0}$, then we have $\mathcal{F}\left[\hat{U}_{2}\right](\cdot, \tau)=\mathcal{S}\left(\lambda_{1}-\gamma+i \tau\right) \mathbf{F}_{\lambda_{1}-\gamma+i \tau}$, where

$$
\mathbf{F}_{\lambda_{1}-\gamma+i \tau}=\left(\mathcal{F}\left[e^{\gamma t} G_{0}\right](\cdot, \tau),\left(\lambda_{1}-\gamma+i \tau\right)^{1 / 2} \mathcal{F}\left[e^{\gamma t} g_{4}\right](\cdot, \tau), \mathcal{F}\left[e^{\gamma t} g_{4}\right](\cdot, \tau)\right) .
$$

Since

$$
\left(\tau \partial_{\tau}\right)^{\ell}\left(i \tau / \lambda_{1}-\gamma+i \tau\right)\left|\leq C_{\lambda_{1}}, \quad\right|\left(\tau \partial_{\tau}\right)^{\ell}\left(\left(\lambda_{1}-\gamma+i \tau\right)^{1 / 2} /\left(1+\tau^{2}\right)^{1 / 4}\right) \mid \leq C_{\lambda_{1}}
$$

for $\ell=0,1$ and $\tau \in \mathbb{R} \backslash\{0\}$, applying Weis' operator valued Fourier multiplier theorem and Bourgain's theorem (cf. Lemma 201) to

$$
U_{1}=\mathcal{F}^{-1}\left[\mathcal{F}\left[U_{1}\right](\cdot, \tau)\right]=\mathcal{F}^{-1}\left[\mathcal{S}\left(\lambda_{1}-\gamma+i \tau\right) \mathbf{F}_{\lambda_{1}-\gamma+i \tau}\right],
$$

we have

$$
\begin{aligned}
& \left\|\partial_{t} U_{2}\right\|_{L_{p}\left(\mathbb{R}, \mathcal{H}_{q}(\Omega)\right)}+\left\|U_{2}\right\|_{L_{p}\left(\mathbb{R}, D_{q}(\Omega)\right)} \\
& \quad \leq C\left(\left\|e^{\gamma t} G_{0}\right\|_{L_{p}\left(\mathbb{R}, \mathcal{H}_{q}(\Omega)\right)}+\left\|e^{\gamma t} g_{4}\right\|_{H_{p}^{1 / 2}\left(\mathbb{R}, L_{q}(\Omega)\right)}+\left\|e^{\gamma t} g_{4}\right\|_{L_{p}\left(\mathbb{R}, H_{q}^{1}(\Omega)\right)}\right) \\
& \quad \leq C\left(\left\|e^{\gamma t} G\right\|_{L_{p}\left((0, T), \mathcal{H}_{q}(\Omega)\right)}+\left\|e^{\gamma t} g_{4}\right\|_{H_{p}^{1 / 2}\left(\mathbb{R}, L_{q}(\Omega)\right)}+\left\|e^{\gamma t} g_{4}\right\|_{L_{p}\left(\mathbb{R}, H_{q}^{1}(\Omega)\right)}\right) .
\end{aligned}
$$

We next consider the Cauchy problem:

$$
\left\{\begin{aligned}
\partial_{t} U_{3}+\left(\lambda_{1}-\gamma\right) U_{3}-P U_{3} & =0 & & \text { in } \Omega \times(0, \infty), \\
B U_{3} & =(0,0) & & \text { on } \Gamma \times(0, \infty), \\
\left.U_{3}\right|_{t=0} & =U_{0}-\left.U_{2}\right|_{t=0} & & \text { in } \Omega .
\end{aligned}\right.
$$

If we choose $\lambda_{1}>0$ sufficiently large, by Theorem 13 we see that there exists a $C^{0}$ analytic semigroup $\left\{T_{1}(t)\right\}_{t \geq 0}$ associated with Eq. (8.22), which is exponentially stable. Setting $U_{3}=T_{1}(t)\left(U_{0}-\left.U_{2}\right|_{t=0}\right)$, we then see that $U_{2}$ satisfies Eq. (8.22) and the estimate:

$$
\left\|e^{\gamma t} \partial_{t} U_{3}\right\|_{L_{p}\left((0, \infty), \mathcal{H}_{q}(\Omega)\right)}+\left\|e^{\gamma t} U_{3}\right\|_{L_{p}\left((0, \infty), D_{q}(\Omega)\right)} \leq C\left\|U_{0}-\left.U_{2}\right|_{t=0}\right\|_{D_{p, q}(\Omega)} .
$$

By the uniqueness of solutions, we have $e^{\gamma t} U_{1}=U_{2}+U_{3}$, and so by (8.25), (8.27), and real interpolation theorem (5.9) and (5.10), we have

$$
\begin{aligned}
& \left\|e^{\gamma t} \partial_{t} U_{1}\right\|_{L_{p}\left((0, T), \mathcal{H}_{q}(\Omega)\right)}+\left\|e^{\gamma t} U_{1}\right\|_{L_{p}\left((0, T), D_{q}(\Omega)\right)} \\
& \quad \leq C\left(\left\|\left(\zeta_{0}, \mathbf{v}_{0}, \vartheta_{0}\right)\right\|_{D_{p, q}(\Omega)}+\left\|e^{\gamma t} G\right\|_{L_{p}\left((0, T), \mathcal{H}_{q}(\Omega)\right)}+\left\|e^{\gamma t} g_{4}\right\|_{H_{p}^{1 / 2}\left(\mathbb{R}, L_{q}(\Omega)\right)}+\left\|e^{\gamma t} g_{4}\right\|_{L_{p}\left(\mathbb{R}, H_{q}^{1}(\Omega)\right)}\right) .
\end{aligned}
$$

We next consider the equations:

$$
\partial_{t} V-P V=-\lambda_{0} U_{1} \quad \text { in } \Omega \times(0, T),\left.\quad B V\right|_{\Gamma}=0,\left.\quad V\right|_{t=0}=0 . \quad \text { in } \Omega .
$$

Let $U_{1}=\left(\zeta_{1}, \mathbf{v}_{1}, \vartheta_{1}\right)$ and set

$$
\tilde{U}_{1}(x, t)=\left(\zeta_{1}(x, t)-\frac{1}{|\Omega|} \int_{\Omega} \zeta_{1}(y, t) d y, \mathbf{v}_{1}(x, t), \vartheta_{1}(x, t)-\frac{1}{|\Omega|} \int_{\Omega} \vartheta_{1}(y, t) d y\right) .
$$


Then $\tilde{U}(\cdot, t) \in \hat{\mathcal{H}}_{q}(\Omega)$ for any $t \in(0, T)$. We consider the equations:

$$
\partial_{t} \tilde{V}-P \tilde{V}=-\lambda_{0} \tilde{U}_{1} \quad \text { in } \Omega \times(0, T),\left.\quad B \tilde{V}\right|_{\Gamma}=0,\left.\quad \tilde{V}\right|_{t=0}=0 . \quad \text { in } \Omega .
$$

In view of (8.18), by the Duhamel principle we have

$$
\tilde{V}=\int_{0}^{t} \dot{T}(t-s) \tilde{U}_{1}(\cdot, s) d s .
$$

Moreover, by (8.19) we have

$$
\left\|e^{\gamma t} \tilde{V}\right\|_{L_{p}\left((0, T), \mathcal{H}_{q}(\Omega)\right)} \leq C\left(\gamma_{1}-\gamma\right)^{-1 / p}\left\|e^{\gamma t} \tilde{U}_{1}\right\|_{L_{p}\left((0, T), \mathcal{H}_{q}(\Omega)\right)} .
$$

Here, we choose $\gamma$ as (8.20). In fact, by (18.19) and Hölder's inequality with exponent $p^{\prime}=p /(p-1)$ we have

$$
\begin{aligned}
e^{\gamma t}\|\tilde{V}(\cdot, t)\|_{L_{q}(\Omega)} & \leq C \int_{0}^{t} e^{\gamma t} e^{-\gamma_{1}(t-s)}\left\|\tilde{U}_{1}(\cdot, s)\right\|_{\mathcal{H}_{q}(\Omega)} d s=C \int_{0}^{t} e^{-\gamma_{1}(t-s)} e^{\gamma s}\left\|\tilde{U}_{1}(\cdot, s)\right\|_{\mathcal{H}_{q}(\Omega)} d s \\
& \leq\left(\int_{0}^{t} e^{-\left(\gamma_{1}-\gamma\right)(t-s)} d s\right)^{1 / p^{\prime}}\left(\int_{0}^{t} e^{-\left(\gamma_{1}-\gamma\right)(t-s)}\left(e^{\gamma s}\|\tilde{U}(\cdot, s)\|_{\mathcal{H}_{q}(\Omega)}\right)^{p} d s\right)^{1 / p},
\end{aligned}
$$

and so by the change of integration order we have

$$
\begin{aligned}
\int_{0}^{T}\left(e^{\gamma t}\|\tilde{V}(\cdot, t)\|_{L_{q}(\Omega)}\right)^{p} d t & \leq C^{p}\left(\gamma_{1}-\gamma\right)^{-p / p^{\prime}} \int_{0}^{T}\left(e^{\gamma s}\|\tilde{U}(\cdot, s)\|_{\mathcal{H}_{q}(\Omega)}\right)^{p} d s \int_{s}^{T} e^{-\left(\gamma_{1}-\gamma\right)(t-s)} d t \\
& =C^{p}\left(\gamma_{1}-\gamma\right)^{-p} \int_{0}^{T}\left(e^{\gamma s}\|\tilde{U}(\cdot, s)\|_{\mathcal{H}_{q}(\Omega)}\right)^{p} d s .
\end{aligned}
$$

Thus, we have (8.32).

Since $\tilde{V}$ satisfies the shifted equations:

$$
\partial_{t} \tilde{V}+\lambda_{0} \tilde{V}-P \tilde{V}=-\lambda \tilde{U}_{1}+\lambda \tilde{V} \quad \text { in } \Omega \times(0, T),\left.\quad B \tilde{V}\right|_{\Gamma}=0,\left.\quad \tilde{V}\right|_{t=0}=0,
$$

we have

$$
\left\|e^{\gamma t} \partial_{t} \tilde{V}\right\|_{L_{p}\left((0, T), \mathcal{H}_{q}(\Omega)\right)}+\left\|e^{\gamma t} \tilde{V}\right\|_{L_{p}\left((0, T), D_{q}(\Omega)\right)} \leq C\left(\left\|e^{\gamma t} \tilde{U}_{1}\right\|_{L_{p}\left((0, T), \mathcal{H}_{q}(\Omega)\right)}+\left\|e^{\gamma t} \tilde{V}\right\|_{L_{p}\left((0, T), \mathcal{H}_{q}(\Omega)\right)}\right),
$$

which, combined with (8.28) and (8.32), leads to

$$
\begin{aligned}
& \left\|e^{\gamma t} \partial_{t} \tilde{V}\right\|_{L_{p}\left((0, T), \mathcal{H}_{q}(\Omega)\right)}+\left\|e^{\gamma t} \tilde{V}\right\|_{L_{p}\left((0, T), D_{q}(\Omega)\right)} \\
& \leq C\left(\left\|\left(\zeta_{0}, \mathbf{v}_{0}, \vartheta_{0}\right)\right\|_{D_{p, q}(\Omega)}+\left\|e^{\gamma t} G\right\|_{L_{p}\left((0, T), \mathcal{H}_{q}(\Omega)\right)}+\left\|e^{\gamma t} g_{4}\right\|_{H_{p}^{1 / 2}\left(\mathbb{R}, L_{q}(\Omega)\right)}+\left\|e^{\gamma t} g_{4}\right\|_{L_{p}\left(\mathbb{R}, H_{q}^{1}(\Omega)\right)}\right) .
\end{aligned}
$$

In view of (8.30), we define $V$ by

$$
V=\tilde{V}-\left(\frac{1}{|\Omega|} \int_{0}^{t} \int_{\Omega} \zeta_{1}(x, s) d x, 0, \frac{1}{|\Omega|} \int_{0}^{t} \int_{\Omega} \vartheta_{1}(x, s) d x d s\right),
$$

and then, $V$ satisfies Eq.(8.29). Moreover, setting $V=\left(\zeta_{2}, \mathbf{v}_{2}, \vartheta_{2}\right)$, by (8.33) and (8.28) we have

$$
\begin{aligned}
& \left\|e^{\gamma t} \partial_{t}\left(\zeta_{2}, \mathbf{v}_{2}, \vartheta_{2}\right)\right\|_{L_{p}\left((0, T), \mathcal{H}_{q}(\Omega)\right)}+\left\|e^{\gamma t} \nabla \zeta_{2}\right\|_{L_{p}\left((0, T), H_{q}^{1}(\Omega)\right)}+\left\|e^{\gamma t} \mathbf{v}_{2}\right\|_{L_{p}\left((0, T), H_{q}^{2}(\Omega)\right)} \\
& \quad+\left\|e^{\gamma t} \nabla \vartheta_{2}\right\|_{L_{p}\left((0, T), H_{q}^{1}(\Omega)\right)} \leq C\left(\left\|\left(\zeta_{0}, \mathbf{v}_{0}, \vartheta_{0}\right)\right\|_{D_{p, q}(\Omega)}+\left\|e^{\gamma t} G\right\|_{L_{p}\left((0, T), \mathcal{H}_{q}(\Omega)\right)}\right. \\
& \left.\quad+\left\|e^{\gamma t} g_{4}\right\|_{H_{p}^{1 / 2}\left(\mathbb{R}, L_{q}(\Omega)\right)}+\left\|e^{\gamma t} g_{4}\right\|_{L_{p}\left(\mathbb{R}, H_{q}^{1}(\Omega)\right)}\right) .
\end{aligned}
$$

Let $(\zeta, \mathbf{v}, \vartheta)=U_{1}+V$, and then $(\zeta, \mathbf{v}, \vartheta)$ is a unique solution of (6.4). Moreover, by (8.34) and (8.28) $(\zeta, \mathbf{v}, \vartheta)$ satisfies the decay estimate:

$$
\begin{aligned}
& \left\|e^{\gamma t} \partial_{t}(\zeta, \mathbf{v}, \vartheta)\right\|_{L_{p}\left((0, T), \mathcal{H}_{q}(\Omega)\right)}+\left\|e^{\gamma t} \nabla \zeta\right\|_{L_{p}\left((0, T), H_{q}^{1}(\Omega)\right)}+\left\|e^{\gamma t} \mathbf{v}\right\|_{L_{p}\left((0, T), H_{q}^{2}(\Omega)\right)}+\left\|e^{\gamma t} \nabla \vartheta\right\|_{L_{p}\left((0, T), H_{q}^{1}(\Omega)\right)} \\
& \leq C\left(\left\|\left(\zeta_{0}, \mathbf{v}_{0}, \vartheta_{0}\right)\right\|_{D_{p, q}(\Omega)}+\left\|e^{\gamma t}\left(g_{1}, \mathbf{g}_{2}, g_{3}\right)\right\|_{L_{p}\left((0, T), \mathcal{H}_{q}(\Omega)\right)}+\left\|e^{\gamma t} g_{4}\right\|_{H_{p}^{1 / 2}\left(\mathbb{R}, L_{q}(\Omega)\right)}+\left\|e^{\gamma t} g_{4}\right\|_{L_{p}\left(\mathbb{R}, H_{q}^{1}(\Omega)\right)}\right) .
\end{aligned}
$$

This completes the proof of Theorem 10, 


\section{References}

[1] H. Amann. Linear and Quasilinear Parabolic Problems. Vol. I. Birkhäuser, Basel, 1995.

[2] J. Bebernes, D. Eberly. Mathematical problems from combustion theory, volume 83 of Applied Mathematical Sciences. Springer-Verlag, New York, 1989.

[3] D. Bothe. On the Maxwell-Stefan approach to multicomponent diffusion. In Parabolic problems, volume 80 of Progr. Nonlinear Differential Equations Appl., pages 81-93. Birkhäuser/Springer Basel AG, Basel, 2011.

[4] D. Bothe, W. Dreyer. Continuum thermodynamics of chemically reacting fluid mixtures. Acta Mech., 226:1757-1805, 2015.

[5] D. Bothe, J Prüss. Modeling and analysis of reactive multi-component two-phase flows with mass transfer and phase transition-the isothermal incompressible case. Discrete Contin. Dyn. Syst. Ser. S 10, no. 4, 673-696, 2017.

[6] J. Bourgain. Vector-valued singular integrals and the $H^{1}-B M O$ duality, In: Probability Theory and Harmonic Analysis, D. Borkholder (ed.) Marcel Dekker, New York, 1-19, 1986.

[7] L. Boudin, B. Grec, and F. Salvarani. A mathematical and numerical analysis of the Maxwell-Stefan diffusion equations. Discrete Contin. Dyn. Syst. Ser. B, 17(5):1427-1440, 2012.

[8] M. Bulicek, J. Havrda. On existence of weak solutions to a model describing compressible mixtures with thermal diffusion cross effects. Z. Angew. Math. Mech. 95, 589-619, 2015.

[9] X. Chen, A. Jüngel. Analysis of an incompressible Navier-Stokes-Maxwell-Stefan system. Comm. Math. Phys., 340 (2), pp. 471-497, 2015.

[10] R. Denk, M. Hieber and J. Prüß. R-boundedness, Fourier multipliers and problems of elliptic and parabolic type. Memoirs of AMS. Vol 166. no. 788, 2003.

[11] W. Dreyer, P-É. Druet, P. Gajewski, and C. Guhlke. Existence of weak solutions for improved Nernst-Planck-Poisson models of compressible reacting electrolytes. preprint WIAS, 2016.

[12] Y. Enomoto, Y. Shibata. On the $\mathcal{R}$-sectoriality and the initial boundary value problem for the viscous compressible fluid flow. Funkcial Ekvac., 56(3), 441-505, 2013.

[13] Y. Enomoto, L. von Below, and Y. Shibata. On some free boundary problem for a compressible barotropic viscous fluid flow. Ann. Univ. Ferrara Sez. VII Sci. Mat., 60(1), 55-89, 2014.

[14] R. Farwig, H. Sohr. Generalized resolvent estimates for the Stokes system in bounded and unbounded domains. J. Math. Soc. Japan, 46 (4), 607-643, 1994.

[15] E. Feireisl, H. Petzeltová, and K. Trivisa. Multicomponent reactive flows: global-in-time existence for large data. Commun. Pure Appl. Anal., 7(5):1017-1047, 2008.

[16] J. Frehse, S. Goj, and J. Málek. A uniqueness result for a model for mixtures in the absence of external forces and interaction momentum. Appl. Math., 50(6):527-541, 2005.

[17] V. Giovangigli. Mass conservation and singular multicomonent diffusion algorithms. IMPACT Comput. Sci. Eng., $2(2): 73-97,1990$.

[18] V. Giovangigli. Convergent iterative methods for multicomponent diffusion. Impact Comput. Sci. Engrg., 3(3):244-276, 1991.

[19] V. Giovangigli. Multicomponent flow modeling. Modeling and Simulation in Science, Engineering and Technology. Birkhäuser Boston Inc., Boston, MA, 1999.

[20] V. Giovangigli, M. Pokorný, and E. Zatorska. On the steady flow of reactive gaseous mixture. Analysis (Berlin) 35, no. 4, 319-341, 2015.

[21] M. Herberg, M. Meyries, J. Prüss, and M. Wilke. Reaction-diffusion systems of Maxwell-Stefan type with reversible mass-action kinetics. Nonlinear Anal. 159, 264-284, 2017.

[22] A. Jüngel. Entropy Methods for Diffusive Partial Differential Equations, SpringerBriefs in Mathematics. Springer 2016.

[23] A. Jüngel, I.V. Stelzer. Existence analysis of Maxwell-Stefan systems for multicomponent mixtures. SIAM J. Math. Anal., 45(4):2421-2440, 2013.

[24] S. Kawashima. Systems of Hyperbolic-Parabolic Composite Type, with Application to the Equations of Magnetohydrodynamics. Doctoral Thesis, Kyoto University, 1984.

[25] S. Kawashima and Y. Shizuta. On the Normal Form of the Symmetric Hyperbolic-Parabolic Systems Associated with the Conservation Laws. Tohoku Math. J., 40, pp. 449-464, 1988.

[26] N. A. Kucher, A. E. Mamontov, and D. A. Prokudin. Stationary solutions to the equations of the dynamics of mixtures of viscous compressible heat-conducting fluids. Sibirsk. Mat. Zh. Sib. Math. J., 53(6):1075-1088, 2012.

[27] M. Marion, R. Temam. Global existence for fully nonlinear reaction-diffusion systems describing multicomponent reactive flows. J. Math. Pures Appl. (9), 104 (1), pp. 102-138, 2015.

[28] A. Matusumura, T. Nishida. Initial-boundary value problems for the equations of motion of compressible viscous and heat-conductive fluids. Comm. Math. Phys. 89 no. 4, 445-464, 1983. 
[29] M. Meyries, R. Schnaubelt. Interpolation, embeddings and traces of aisotropic fractional Sobolev spaces with temporal weights, J. Func. Anal. 262, 1200-1229, 2012.

[30] P. B. Mucha, M. Pokorný, and E. Zatorska. Approximate solutions to model of two-component reactive flow. Discrete Contin. Dyn. Syst. Ser. S, 7, no.5, 1079-1099, 2014.

[31] P. B. Mucha, M. Pokorný, and E. Zatorska. Chemically reacting mixtures in terms of degenerated parabolic setting. J. Math. Phys., 54(071501), 2013.

[32] P. B. Mucha, M. Pokorný, and E. Zatorska. Heat-conducting, compressible mixtures with multicomponent diffusion: construction of a weak solution. SIAM J. Math. Anal. 47, no. 5, 3747-3797, 2015.

[33] M. Murata. On a maximal $L_{p}-L_{q}$ approach to the compressible viscous fluid flow with slip boundary condition. Nonlinear Analysis, 106, 86-109, 2014.

[34] M. Murata, Y. Shibata. On the global well-posedness for the compressible Navier-Stokes equations with slip boundary condition, J. Differential Equtions 260 (7), 5761-5795, 2016.

[35] T. Piasecki, M. Pokorný. Weak and variational entropy solutions to the system describing steady flow of a compressible reactive mixture. Nonlinear Anal. 159, 365-392, 2017.

[36] T. Piasecki, M. Pokorný. On steady solutions to a model of chemically reacting heat conducting compressible mixture with slip boundary conditions Preprint, arXiv:1709.06886, 2017.

[37] K. Schade, Y. Shibata. On strong dynamics of compressible Nematic Liquid Crystals, SIAM J. Math. Anal. 47(5), 3963-3992, 2015.

[38] Y. Shibata. On the global well-posedness of some free boundary problem for a compressible barotropic viscous fluid flow. Recent advances in partial differential equations and applications, 341356, Contemp. Math., 666, Amer. Math. Soc., Providence, RI, 2016.

[39] Y. Shibata. On the local wellposedness of free boundary problem for the Navier-Stokes equations in an exterior domain, Submitted.

[40] Y. Shibata and S. Shimizu. On some free boundary problem for the Navier-Stokes equtions, Diff. Int. Eqns., 20, 241-276, 2007.

[41] Y. Shibata, S. Shimizu. On the $L_{p}-L_{q}$ maximal regularity of the Neumann problem for the Stokes equations in a bounded domain, J. Reine Angew. Mat., 615, 157-209, 2008.

[42] Y. Shibata, K. Tanaka. On a resolvent problem for the linearized system from the dynamical system describing the compressible viscous fluid motion, Math. Mech. Appl. Sci. 27, 1579-1606, 2004.

[43] G. Ströhmer. About a certain class of parabolic-hyperbolic systems of differential equation, Analysis 9, 1-39, 1989.

[44] H. Tanabe. Functional analytic methods for partial diffeential equations, Monographs and textbooks in pure and applied mathematics, Vol 204, Marchel Dekker, Inc. New York, Basel, 1997.

[45] S. R. Turns. An introduction to combustion: Concepts and applications. McGraw-Hill Series in Mechanical Engineering, 2000.

[46] L. Waldmann, E. Trübenbacher. Formale kinetische Theorie von Gasgemischen aus anregbaren Molekülen. Z. Naturforsch, 17a:363-376, 1962.

[47] L. Weis. Operator-valued Fourier multiplier theorems and maximal $L_{p}$-regularity. Math. Ann. 319, 735-758, 2001.

[48] W. M. Zajaczkowski. On nonstationary motion of a compressible barotropic viscous fluid with boundary slip condition. J. Appl. Anal. 4(2):167-204, 1998.

[49] E. Zatorska. On a steady flow of multicomponent, compressible, chemically reacting gas. Nonlinearity, 24:3267-3278, 2011.

[50] E. Zatorska. On the flow of chemically reacting gaseous mixture. J. Differential Equations, 253(12):3471-3500, 2012.

[51] E. Zatorska. Mixtures: sequential stability of variational entropy solutions. J. Math. Fluid Mech. 17, no. 3, 437-461, 2015.

[52] X.Xi, B. Xie. Global existence of weak solutions for the multicomponent reaction flows. Journal of Mathematical Analysis and Applications Volume 441, Issue 2, 15 September 2016, Pages 801-814. 